\title{
Hydroxytyrosol. A versatile antioxidant from olive oil
}

Citation for published version (APA):

Rietjens, S. J. (2008). Hydroxytyrosol. A versatile antioxidant from olive oil. [Doctoral Thesis, Maastricht University]. Maastricht University. https://doi.org/10.26481/dis.20080918sr

Document status and date:

Published: 01/01/2008

DOI:

10.26481/dis.20080918sr

Document Version:

Publisher's PDF, also known as Version of record

\section{Please check the document version of this publication:}

- A submitted manuscript is the version of the article upon submission and before peer-review. There can be important differences between the submitted version and the official published version of record.

People interested in the research are advised to contact the author for the final version of the publication, or visit the DOI to the publisher's website.

- The final author version and the galley proof are versions of the publication after peer review.

- The final published version features the final layout of the paper including the volume, issue and page numbers.

Link to publication

\footnotetext{
General rights rights.

- You may freely distribute the URL identifying the publication in the public portal. please follow below link for the End User Agreement:

www.umlib.nl/taverne-license

Take down policy

If you believe that this document breaches copyright please contact us at:

repository@maastrichtuniversity.nl

providing details and we will investigate your claim.
}

Copyright and moral rights for the publications made accessible in the public portal are retained by the authors and/or other copyright owners and it is a condition of accessing publications that users recognise and abide by the legal requirements associated with these

- Users may download and print one copy of any publication from the public portal for the purpose of private study or research.

- You may not further distribute the material or use it for any profit-making activity or commercial gain

If the publication is distributed under the terms of Article $25 \mathrm{fa}$ of the Dutch Copyright Act, indicated by the "Taverne" license above, 


\section{HYDROXYTYROSOL}

A VERSATILE ANTIOXIDANT FROM OLIVE OIL 
(C) Saskia J. Rietjens, Maastricht 2008

ISBN 978-90-5278-738-1

Cover design: Jantine Slinger

Production: Datawyse / Universitaire Pers Maastricht

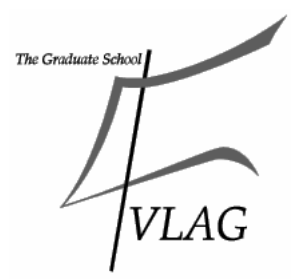

The studies presented in this thesis were performed within the Nutrition and Toxicology Research Institute Maastricht (NUTRIM) which participates in the Graduate School VLAG (Food Technology, Agrobiotechnology, Nutrition and Health Sciences), accredited by the Royal Netherlands Academy of Arts and Sciences. 


\section{HYDROXYTYROSOL}

\section{A VERSATILE ANTIOXIDANT FROM OLIVE OIL}

\section{Proefschrift}

ter verkrijging van de graad van doctor aan de Universiteit Maastricht, op gezag van de Rector Magnificus, Prof. mr. G.P.M.F. Mols volgens het besluit van het College van Decanen, in het openbaar te verdedigen op donderdag 18 september om 12.00 uur

door

\section{Saskia Johannes Rietjens}

geboren te Roermond op 12 juli 1980

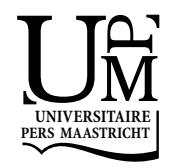




\section{Promotor}

Prof. dr. A. Bast

\section{Copromotor}

Dr. G.R.M.M Haenen

\section{Beoordelingscommissie}

Prof. dr. A.M. Schols (voorzitter)

Prof. dr. G. Bartosz (University of Lodz, Poland)

Dr. R. Beudeker (DSM Food Specialties, Delft)

Prof. dr. H. Kuipers

Prof. dr. W.H. Saris 
Mooi zijn de stromen die komen uit de bron van het hart

(Bert Schierbeek) 



\section{CONTENTS}

$\begin{array}{lr}\text { List of abbreviations } & 8\end{array}$

$\begin{array}{lll}\text { Chapter } 1 \text { General introduction } & 11\end{array}$

Chapter 2 New insights into controversies on the antioxidant potential $\quad 27$ of the olive oil antioxidant hydroxytyrosol

Chapter 3 The olive oil antioxidant hydroxytyrosol efficiently protects against the oxidative stress-induced impairment of the $\mathrm{NO}^{\bullet}$ response of isolated rat aorta

Chapter 4 A single session of resistance exercise induces oxidative damage in untrained men

Chapter 5 The olive oil antioxidant hydroxytyrosol protects against oxidative stress-induced reduction in skeletal muscle force production

Chapter 6 The olive antioxidant hydroxytyrosol boosts the endogenous antioxidant defence and attenuates the rise in plasma lactate during resistance type exercise

Chapter 7 General discussion

Chapter 8 Summary and perspective

Appendix Dankwoord

About the author

Publications 


\section{LIST OF ABBREVIATIONS}

$\begin{array}{ll}\text { 1RM } & \text { one repetition maximum } \\ \text { 8-iso-PGF } & \text { 8-isoprostane } \\ \text { ARE } & \text { antioxidant responsive element } \\ \text { AUC } & \text { area under the curve } \\ \text { CAT } & \text { catalase } \\ \text { CCh } & \text { carbachol } \\ \text { cGMP } & \text { cyclic guanosine monophosphate } \\ \text { CHD } & \text { coronary heart disease } \\ \text { CHP } & \text { cumene hydroperoxide } \\ \text { COPD } & \text { chronic obstructive pulmonary disease } \\ \text { CuZnSOD } & \text { copper-zinc superoxide dismutase } \\ \text { DTNB } & \text { 5,5'-dithiobis-2-nitrobenzoic acid } \\ \text { ELISA } & \text { enzyme-linked immunosorbent assay } \\ \mathrm{E}_{\text {max }} & \text { maximal effect } \\ \text { eNOS } & \text { endothelial nitric oxide synthase } \\ \text { GC } & \text { guanylyl cyclase } \\ \text { GPX } & \text { glutathione peroxidase } \\ \text { GSH } & \text { glutathione } \\ \text { GSSG } & \text { glutathione disulphide } \\ \text { GST } & \text { glutathione S-transferase } \\ \text { GTP } & \text { guanosine triphosphate } \\ \mathrm{H}_{2} \mathrm{O}_{2} & \text { hydrogen peroxide } \\ \mathrm{HOCl} & \text { hypochlorous acid } \\ \mathrm{HDL} & \text { high density lipoprotein } \\ \text { LDH } & \text { lactate dehydrogenase } \\ \text { IL-6 } & \text { interleukin-6 } \\ \text { IL-10 } & \text { interleukin-10 } \\ \text { LDL } & \text { low density lipoprotein } \\ \mathrm{L}_{\mathrm{o}} & \text { optimal length } \\ \text { LOOH } & \text { lipid hydroperoxide } \\ \text { LPS } & \text { lipopolysaccharide } \\ \mathrm{MAPK} & \text { mitogen-activated protein kinase } \\ \mathrm{MDA} & \text { malondialdehyde } \\ \mathrm{MnSOD} & \text { manganese superoxide dismutase } \\ \mathrm{MUFA} & \text { mono-unsaturated fatty acid } \\ \mathrm{NAC} & \text { N-acetylcysteine } \\ \mathrm{NADPHox} & \text { nicotinamide adenine dinucleotide phosphate oxidase } \\ \mathrm{NF-KB} & \text { nuclear factor kappa B } \\ \mathrm{NO} & \text { nitric oxide radical } \\ \mathrm{O}_{2}{ }^{*} & \text { superoxide radical } \\ & \end{array}$




$\begin{array}{ll}\mathrm{OH}^{\cdot} & \text { hydroxyl radical } \\ \text { OMWW } & \text { olive oil mill wastewater } \\ \text { ONOOH } & \text { peroxynitrite } \\ \text { PBMC } & \text { peripheral blood mononuclear cell } \\ \mathrm{pD}_{2} & \text { negative logarithm of the molar concentration that produces } \\ & \text { half-maximal effect } \\ \mathrm{PE} & \text { phenylephrine } \\ \mathrm{P}_{\mathrm{o} / \mathrm{w}} & \text { octanol water partitioning coefficient } \\ \text { PUFA } & \text { poly-unsaturated fatty acid } \\ \text { RNS } & \text { reactive nitrogen species } \\ \text { RONS } & \text { reactive oxygen and nitrogen species } \\ \text { ROS } & \text { reactive oxygen species } \\ \text { RYR } & \text { ryanodine receptor } \\ \text { SOD } & \text { superoxide dismutase } \\ \text { SSA } & \text { sulphosalicylic acid } \\ \mathrm{t}_{1 / 2} & \text { half life } \\ \text { TBARS } & \text { thiobarbituric acid-reacting substances } \\ \text { TBS } & \text { tris-buffered saline } \\ \text { TBS-T } & \text { tris-buffered saline containing Triton X-100 } \\ \text { TCA } & \text { trichloroacetic acid } \\ \text { TEAC } & \text { trolox equivalent antioxidant capacity } \\ \text { TNF } \alpha & \text { tumor necrosis factor alpha } \\ X & \text { xanthine } \\ \text { XO } & \text { xanthine oxidase } \\ & \end{array}$





\section{CHAPTER 1}

\section{General introduction}

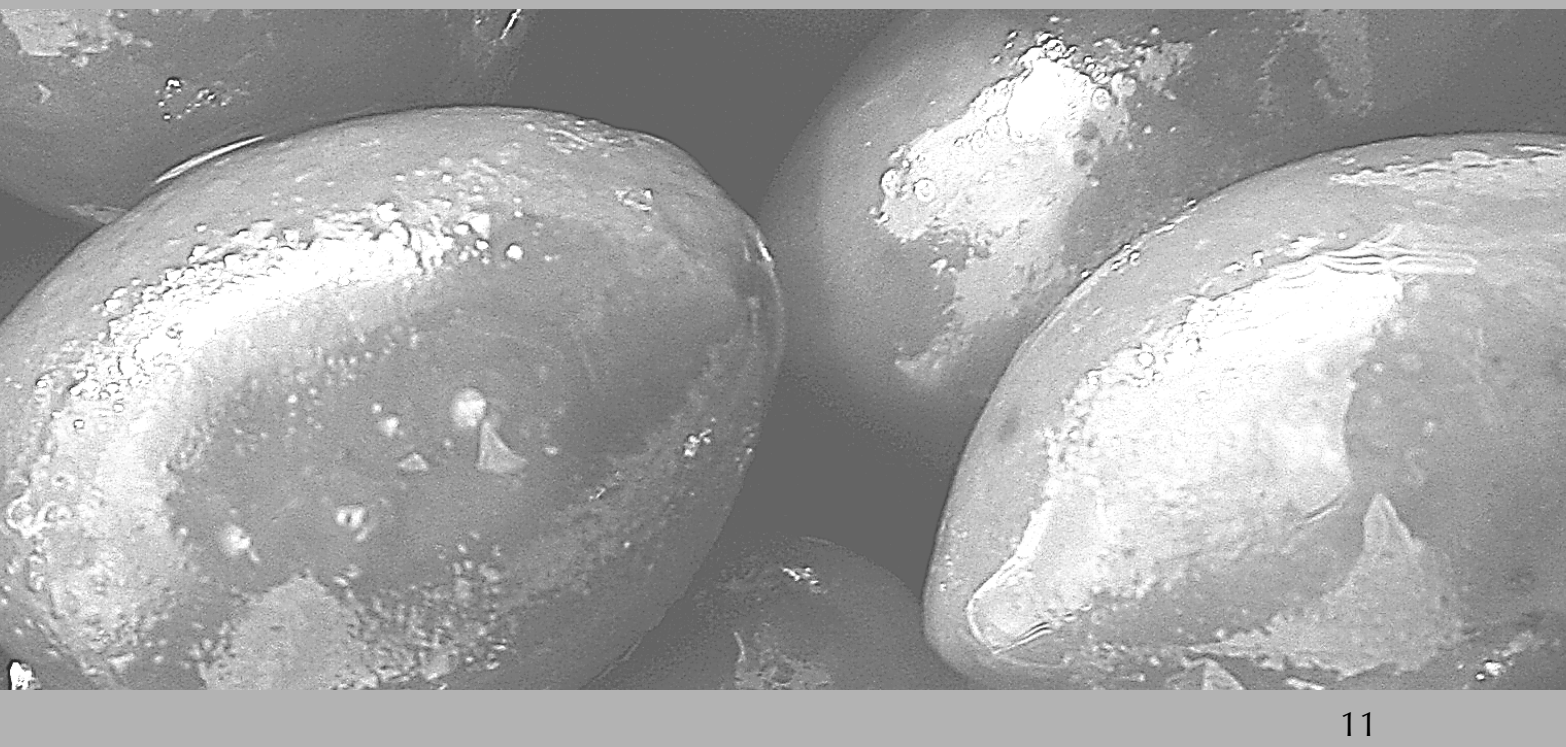




\section{OLIVE OIL}

\section{Origin of olive oil}

Olive oil is obtained from the fruits of Olea Europea, a traditional tree crop of the Mediterranean Basin. The annual worldwide olive oil production is estimated at about 2,000,000,000 kg $(1,2)$. It can be calculated that, when all bottles of olive oil annually produced are placed in a row, this would correspond with a distance of approximately 15 times the circumference of the earth.

The Mediterranean countries supply about 95\% of the world olive oil production, $75 \%$ of which comes from the European Union (mostly Spain, Italy and Greece) (2). The production is now expanding to non-traditional producers such as the USA, South America and Australia.

\section{Olive oil consumption}

Greece, Spain and Italy have the highest per capita consumption of olive oil worldwide, ranging from 10-20 kg per capita per year (3). The average intake of olive oil is highest in Greece, i.e. approximately $54 \mathrm{~g}$ per capita per day. In contrast, the consumption of olive oil is much lower in Northern and Western European countries. For example, in Denmark the average consumption of olive oil is only $1 \mathrm{~g}$ per capita per day (3).

Consumption of olive oil is increasing in non-Mediterranean areas such as the USA and Australia. This is due to the growing interest in the health benefits of the Mediterranean diet, of which olive oil is the principal source of fat.

\section{Composition and classification of olive oil}

Olive oil is abundant in oleic acid, a mono-unsaturated fatty acid (MUFA) (18:1n-9), which ranges from 55 to $83 \%$ of total fatty acids, while the polyunsaturated fatty acid (PUFA) linoleic acid (18:2n-6) is present in concentrations between 3 and $21 \%$ (4).

Depending on its degree of acidity, olive oil is classified into different grades that serve as guidelines for the consumer in the choice of the preferred kind of oil. The oil's acidity, which is determined by its free oleic acid content, is a measure of the degradation of the oil.

The most valuable kind of olive oil is the extra-virgin one, obtained from intact olives that are quickly processed and cold-pressed without additional treatment. In this way, degradation of triglycerides is minimized. Extra-virgin olive oil has a maximum acidity of no more than $1 \%$ ( $1 \mathrm{~g}$ free oleic acid per $100 \mathrm{~g}$ oil). Virgin olive oil is a somewhat lower quality type of olive oil; its acidity 
may not exceed $1.5 \%$. Refined olive oil means that the oil has been treated to neutralize the acid content and is commonly regarded as a lower quality type of olive oil, compared to (extra) virgin olive oil. Extra-virgin olive oil is judged to have a superior taste, compared to virgin and refined olive oil.

(Extra) virgin olive oil contains several minor constituents such as vitamin $\mathrm{E}$, $\beta$-carotene, flavonoids and other phenolic compounds.

\section{OLIVE OIL PHENOLIC COMPOUNDS}

\section{Olive oil phenolic content}

The amount of phenolic compounds in olive oil depends on several factors, including cultivar, climate, degree of maturation of the olives, manufacturing process, and storage conditions. The major phenolic compounds present in olive oil are hydroxytyrosol, tyrosol and oleuropein (Figure 1).<smiles>OCCc1ccc(O)c(O)c1</smiles>

hydroxytyrosol<smiles>OCCc1ccc(O)cc1</smiles>

tyrosol<smiles>CC=C1C(OC(C)C)OC=C(C(=O)OC)C1CC(=O)OCCc1ccc(O)c(O)c1</smiles>

oleuropein<smiles>COc1cc(CCO)ccc1O</smiles>

homovanillic alcohol

Figure 1. Molecular structure of hydroxytyrosol, oleuropein, tyrosol and homovanillic alcohol.

The total concentration of phenolic compounds in extra-virgin olive oil has been reported to vary between 50 and $800 \mathrm{mg} / \mathrm{kg}$ (5). Owen et al. reported a mean concentration of phenolic compounds of $230 \mathrm{mg} / \mathrm{kg}$ in extra virgin olive oil (6). In refined olive oil, the mean phenolic content is significantly lower (6). The amount of oleuropein in olive oil is relatively small. However, relatively high amounts of oleuropein are present in olives (4). 


\section{Olive oil mill wastewater (OMWW)}

During the production of olive oil, large quantities of OMWW are produced. It is estimated that approximately 2,000,000,000 litre of OMWW is annually produced in Greece, currently disposed in evaporation tanks during the olive oil production season (1). This corresponds with approximately $40 \%$ of the annual domestic consumption of drinking water in Maastricht, the Netherlands (approximately 120,000 inhabitants).

OMWW has a higher content of phenolic compounds than olive oil itself. Concentrations up to 4,000 mg/l (7) and 11,000 mg/l (8) of total polyphenols in OMWW have been reported. The amount of antioxidants in olive oil is only 1 $2 \%$ of the available pool of antioxidants in the olive fruit. The remainder is lost either in OMWW (approximately 53\%) or in the pomace (approximately 45\%) (1).

OMWW constitutes a serious environmental problem, as it has severe negative impact on soil and water quality $(8,9)$. In addition to the large volume of OMWW produced, the high content of phenolic compounds is regarded as one of the major factors of the environmental problems imposed by OMWW (1).

\section{Recovery of phenolic compounds from OMWW}

The recovery of phenolic compounds from OMWW is very valuable, not only from an environmental point of view, but also because these compounds might be useful in the pharmaceutical, food and cosmetic industry. Techniques are being optimized to extract phenolic compounds from OMWW $(1,8)$. Supplements with health promoting claims are currently being developed from OMWW. The content of phenolic compounds present in some of these extracts is 300-500 times higher than in olive oil itself. In this way, a waste pollutant is turned into a health promoting product.

\section{Beneficial cardiovascular effects of olive oil phenolic compounds}

Epidemiological evidence has shown that the incidence of coronary heart disease (CHD) is lower in Mediterranean countries than in Western-European and Northern-European countries (10). The Mediterranean diet is thought to significantly contribute to the protection against CHD. The main characteristics of the traditional Mediterranean diet include an abundance of plant foods (fruits, vegetables, whole-grain cereals, nuts and legumes), a high consumption of olive oil, a low-to-moderate consumption of fish and poultry, a low consumption of red meat, and a moderate consumption of wine (11) (Figure 2). 


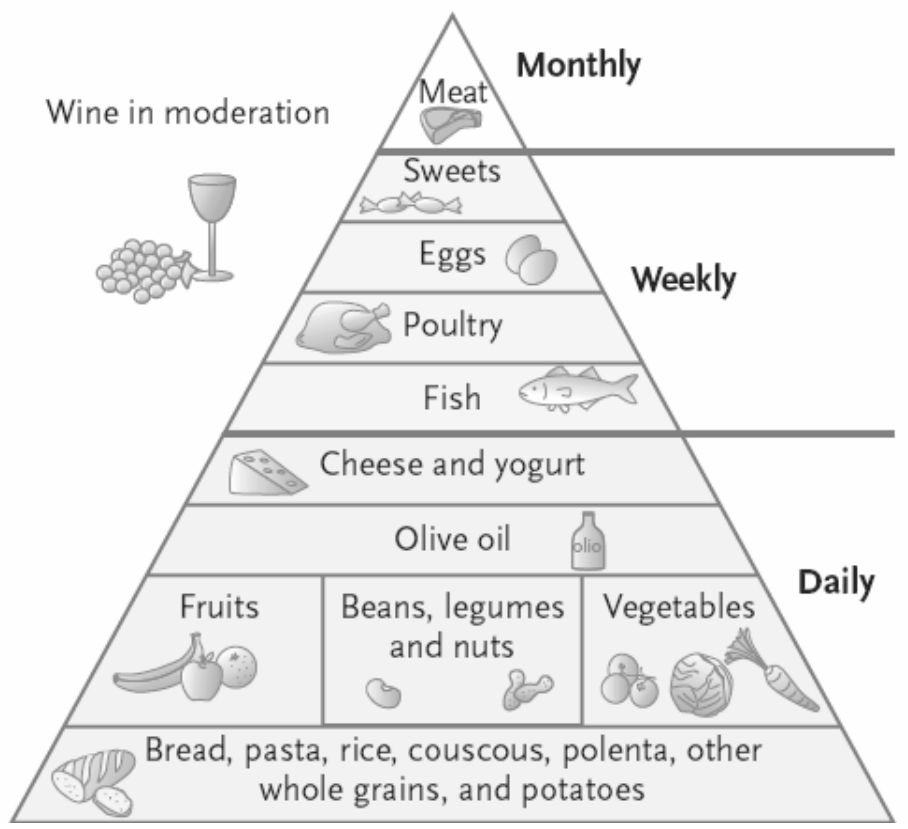

Figure 2. Olive oil is the principal source of fat and one of the key components of the traditional Mediterranean diet.

Adapted from Hu et al. N Engl J Med. 2003;348(26):2595-6.

Of the components of the Mediterranean diet, in particular olive oil has gained a lot of interest. The consumption of olive oil has been shown to provide cardiovascular health benefits such as favourable effects on cholesterol regulation and low density lipoprotein (LDL) oxidation (12). The beneficial effects of olive oil on CHD are often attributed to its high MUFA content. However, phenolic compounds present in virgin olive oil have been shown to provide additional beneficial cardiovascular effects (13-16).

\section{ANTIOXIDANT PROPERTIES OF OLIVE OIL}

Of the phenolic compounds present in olive oil and OMWW, especially hydroxytyrosol has gained a lot of attention. Research on hydroxytyrosol is particularly focused on its antioxidant properties, such as the scavenging potential of reactive oxygen and nitrogen species (RONS).

\section{Reactive oxygen and nitrogen species (RONS)}

RONS are continuously produced in the body and function as essential mediators in several vital processes including smooth muscle relaxation and the 
respiratory burst to kill invading micro-organisms. Physiological important reactive oxygen species (ROS) include superoxide radical $\left(\mathrm{O}_{2}{ }^{-}\right)$, hydroxyl radical $\left(\mathrm{OH}^{*}\right)$, hydrogen peroxide $\left(\mathrm{H}_{2} \mathrm{O}_{2}\right)$, and hypochlorous acid $(\mathrm{HOCl})$. Examples of physiological important reactive nitrogen species (RNS) include nitric oxide $\left(\mathrm{NO}^{*}\right)$ and peroxynitrite $(\mathrm{ONOOH})$. The reactivity of $\mathrm{H}_{2} \mathrm{O}_{2}$ itself is relatively low. However, $\mathrm{H}_{2} \mathrm{O}_{2}$ can be converted to other reactive species, such as $\mathrm{OH}^{\circ}$.

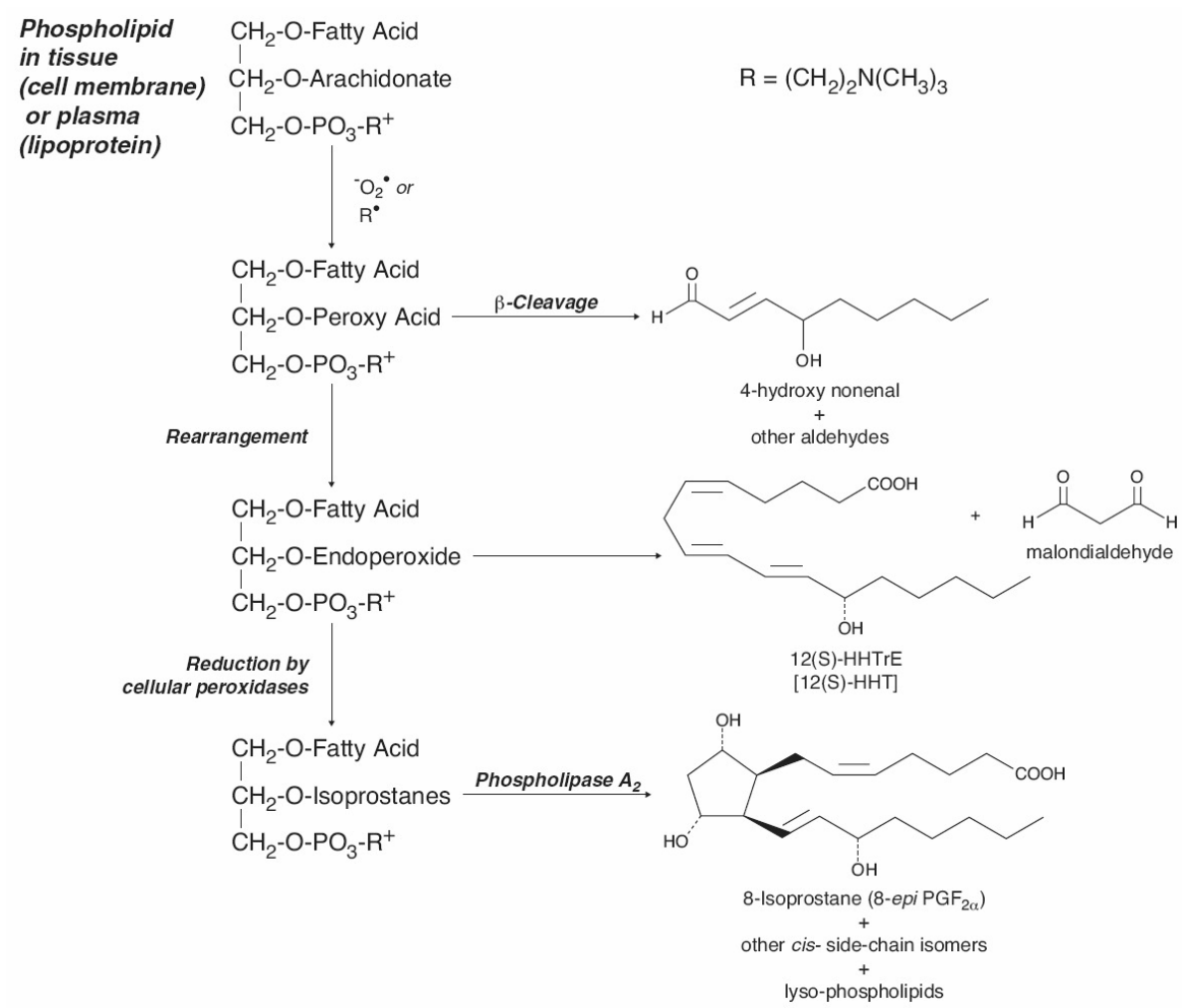

Figure 3. The formation of 8-isoprostane.

Adapted from Cayman Chemical.

PUFAs, present for example in cell membranes, are important targets of oxidation by RONS (17). Initiation of this reaction involves the subtraction of a hydrogen atom from the attacked PUFA, thereby leaving an unpaired electron on the lipid molecule. This newly-formed lipid radical will rapidly react with oxygen, creating a peroxyl radical. Subsequently, this peroxyl radical will be converted into a lipid hydroperoxide and generate a new lipid radical by subtracting a hydrogen atom from a second PUFA. This chain reaction, referred to as lipid peroxidation, takes place by continuously passing the unpaired 
electron from one molecule to another. The free radical chain reaction will continue to propagate until quenched by an antioxidant. In Figure 3 is shown the formation of 8-isoprostane (8-iso-PGF $2 a$ ) from arachidonic acid during peroxidation. It has been found that 8 -iso- $\mathrm{PGF}_{2 \alpha}$ is a good biomarker for lipid peroxidation.

Besides lipid peroxidation, damage caused by RONS might also lead to DNA lesions, functional loss of enzymes, disturbed cell signalling, and, eventually, necrotic cell death or apoptosis.

\section{Protection against RONS}

Fortunately, the body comprises an elaborate antioxidant defence system which provides protection against RONS. The antioxidant system can be subdivided into enzymatic and non-enzymatic antioxidants. Important enzymatic antioxidants are superoxide dismutase (SOD), catalase (CAT) and glutathione peroxidase (GPX). SOD catalyzes the dismutation of $\mathrm{O}_{2}{ }^{--}$to $\mathrm{H}_{2} \mathrm{O}_{2}$ and oxygen $\left(\mathrm{O}_{2}\right)$. CAT and GPX catalyze the conversion of $\mathrm{H}_{2} \mathrm{O}_{2}$ into $\mathrm{H}_{2} \mathrm{O}$. Important nonenzymatic antioxidants include the hydrophilic vitamin $\mathrm{C}$ and glutathione $(\mathrm{GSH})$, and the hydrophobic vitamin $\mathrm{E}$.

In case of a diet rich in vegetables and fruits, the endogenous antioxidant system provides sufficient protection against RONS (18). However, when the production of RONS overwhelms the protection provided by antioxidants, a situation called oxidative stress arises. Oxidative stress is associated with the pathophysiology of various diseases, e.g. cardiovascular diseases (19) and lung diseases such as chronic obstructive pulmonary disease (COPD) (20).

The supplementation of exogenous, dietary antioxidants might reduce oxidative stress and associated damage. Hydroxytyrosol might be an interesting compound to empower the antioxidant defence system in situations of oxidative stress. Important aspects that should be considered regarding the use of hydroxytyrosol as a supplement include not only its antioxidant potency, but also its bioavailability.

\section{Antioxidant properties of hydroxytyrosol in vitro}

Several studies on antioxidant properties of hydroxytyrosol have been performed. Deiana et al. have shown that hydroxytyrosol is able to scavenge $\mathrm{ONOOH}$ and therefore prevents $\mathrm{ONOOH}$ dependent DNA damage and tyrosine nitration (21). These results are in accordance with those of de la Puerta et al. who have also shown that hydroxytyrosol is able to scavenge $\mathrm{ONOOH}$ (22). Regarding the $\mathrm{O}_{2}{ }^{-}$radical scavenging potential of hydroxytyrosol some controversies exist $(23,24)$. Hydroxytyrosol has been shown to provide protection against oxidative stress-induced damage to several types of 
cells, such as intestinal epithelial cells (25), erythrocytes (26) and hepatocytes (27).

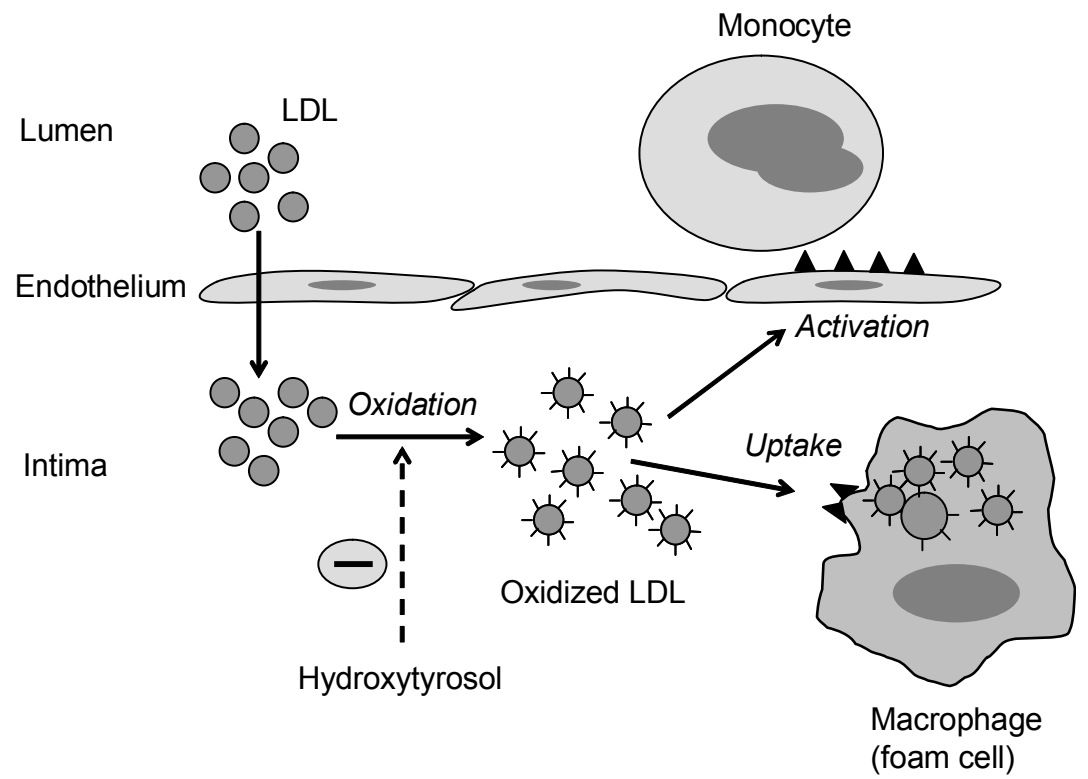

Figure 4. LDL oxidation and uptake of oxidized LDL in macrophage, one of the key steps in the initiation of atherosclerosis. Oxidation of LDL can activate endothelial cells to express leukocyte adhesion molecules. This causes blood cells such as monocytes rolling along the vascular surface to adhere to the site of activation. Monocytes enter the intima and turn into macrophages. The modified LDL particles are taken up by scavenger receptors of macrophages, which evolve into foam cells. Hydroxytyrosol prevents the oxidation of LDL and thereby inhibits the formation of foam cells.

Furthermore, it has been reported that hydroxytyrosol efficiently protects against lipid peroxidation in vitro (28). The protection against lipid peroxidation by hydroxytyrosol is also shown by the inhibition of the oxidation of LDL, which is regarded as one of the key steps in the initiation of atherosclerosis (29, 30). Excess LDL infiltrates the artery and is retained in the intima, particularly at sites of haemodynamic strain. Oxidation of LDL can activate endothelial cells to express leukocyte adhesion molecules. This causes blood cells such as monocytes rolling along the vascular surface to adhere to the site of activation. The modified LDL particles are taken up by scavenger receptors of macrophages, which evolve into foam cells (Figure 4). Activation of macrophages leads to an inflammatory reaction and tissue damage (31).

\section{Antioxidant properties of hydroxytyrosol in vivo}

For an antioxidant to have beneficial health effects in vivo it is of course essential that it is taken up. Several studies in humans and rats indeed report 
that the uptake of hydroxytyrosol is good (32-35). Plasma concentrations of hydroxytyrosol after consumption of $25 \mathrm{ml}$ of extra virgin olive oil range from 50 to $160 \mathrm{nM}(16,36)$. The consumption of supplements containing a relatively high amount of hydroxytyrosol might lead to an even higher hydroxytyrosol plasma concentration.

Hydroxytyrosol is metabolized in the body by the action of catechol-Omethyltransferase to homovanillic alcohol (Figure 1). Ingested oleuropein, one of the other phenolic compounds also present in virgin olive oil, is hydrolyzed in the intestine yielding additional hydroxytyrosol. Oleuropein itself hardly reaches the systemic circulation after ingestion $(37,38)$.

Visioli et al. have shown that hydroxytyrosol was able to inhibit passive smoking-induced oxidative stress in rats, as demonstrated by a reduced urinary excretion of isoprostanes (8-iso-PGF ${ }_{2 \alpha}$ ) (39). Moreover, a dose-dependent inverse correlation between the rate of 8 -iso- $\mathrm{PGF}_{2 \alpha}$ excretion and increasing amounts of phenolic compounds ingested with olive oil was observed in human volunteers (40). Furthermore, hydroxytyrosol was able to increase plasma antioxidant capacity in rats (33).

Regarding the protective effect of hydroxytyrosol against the oxidation of LDL some controversies exist. Several human in vivo studies have shown that olive oil phenolic compounds efficiently protect against LDL oxidation (13-16, 41). However, no protective effect of hydroxytyrosol is usually found against LDL oxidation in ex vivo experiments (42-45).

\section{HYDROXYTYROSOL: SCOPE AND OUTLINE OF THE THESIS}

In general, hydroxytyrosol supplementation might be of interest in all kinds of situations of oxidative stress. The present thesis focuses on two specific aspects of hydroxytyrosol. Firstly, one of the 'traditional' fields of interest of hydroxytyrosol, i.e. its beneficial effects on the cardiovascular system, is further explored. Secondly, the possible beneficial effect of hydroxytyrosol is studied in an innovative, so far unexplored field of research, i.e. its protective effect against oxidative stress in skeletal muscle.

\section{Effect of hydroxytyrosol on cardiovascular system}

The molecular mechanism of the beneficial cardiovascular effects of olive oil antioxidants has not been fully elucidated. Studies investigating the antioxidant effects of olive oil phenolic compounds are conflicting (5). It has been shown in both in vitro and human in vivo studies that phenolic compounds efficiently protect against LDL oxidation (13-16, 29, 30, 41, 46). In contrast, no protective effect of hydroxytyrosol is generally demonstrated against ex vivo LDL oxida- 
tion (42-45). In these experiments LDL is isolated from humans after hydroxytyrosol consumption, and subsequently the oxidizability of this LDL is determined ex vivo. Also regarding the antioxidant profile of hydroxytyrosol some controversies exist. For example, the reports on $\mathrm{O}_{2}{ }^{-}$radical scavenging are ambiguous $(23,24)$. Chapter 2 is aimed at resolving the controversies on the antioxidant profile of hydroxytyrosol and on the protective effect of hydroxytyrosol against LDL oxidation. Moreover, the scavenging potential of hydroxytyrosol and related compounds is also further explored.

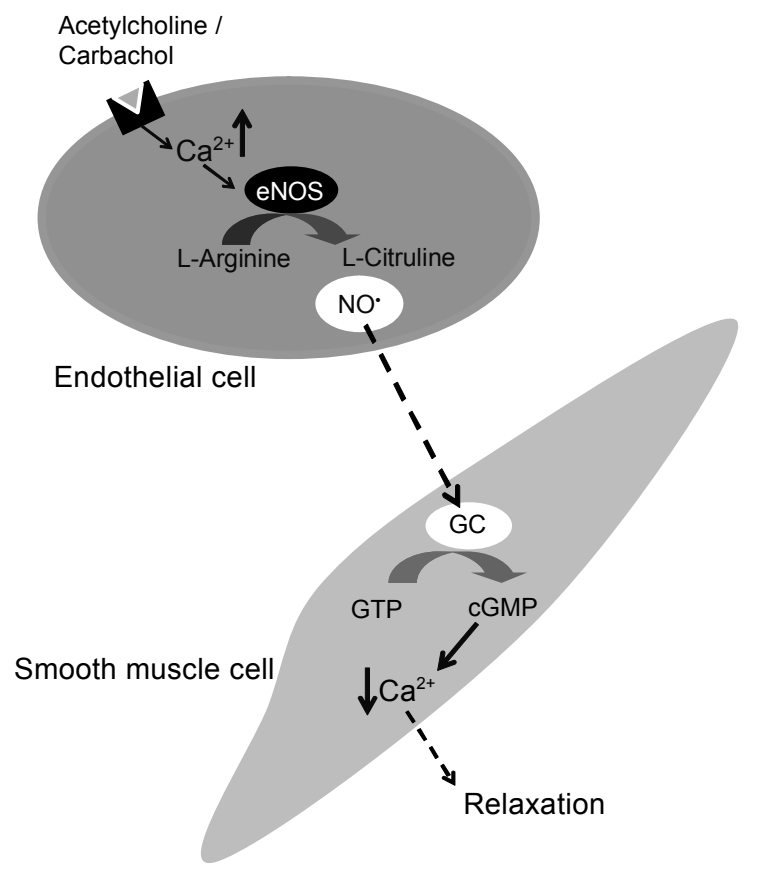

Figure 5. $\mathrm{NO}^{\bullet}$ mediated vasorelaxation. Biosynthesis of $\mathrm{NO}^{\bullet}$ is performed by the calcium dependent endothelial isoform of nitric oxide synthase (eNOS). The binding of an agonist triggers the conversion of L-Arginine by eNOS into $\mathrm{NO}^{\bullet}$, with L-Citruline as byproduct. $\mathrm{NO}^{\bullet}$ diffuses into adjacent smooth muscle cells where it activates guanylyl cyclase (GC). GC converts guanosine triphosphate (GTP) into the second messenger cyclic guanosine monophosphate (cGMP), which activates protein kinases (PKG) leading to blood vessel relaxation.

Under physiological conditions, $\mathrm{NO}^{\bullet}$ is of pivotal importance in the regulation of the vascular tone. Endothelium-derived $\mathrm{NO}^{\bullet}$ stimulates soluble guanylyl cyclase (GC) activity in smooth muscle cells, finally leading to vasorelaxation (Figure 5). Oxidative damage to endothelial cells might lead to hypertension, which is a well-established risk factor for the development or progression of atherosclerosis $(47,48)$. Chapter 3 describes the protective effect of hydroxytyrosol against the oxidative stress induced impairment of the $\mathrm{NO}^{\bullet}$ mediated vasorelaxation of rat aorta. 


\section{Effect of hydroxytyrosol on skeletal muscle}

The production of ROS is significantly enhanced during exercise. For example, superoxide radical $\left(\mathrm{O}_{2}{ }^{\circ}\right)$ production by mitochondria is substantially increased (49). Other potential sources for ROS generation during exercise are plasma membrane NADPH oxidases and ischemia reperfusion (50-52). Moreover, ROS trigger the activation of pro-inflammatory pathways such as Nuclear Factor kappa B (NF-kB) and the invasion of the injured tissue with phagocytic cells. These pathways further promote the production of ROS in a feed forward process (Figure 6).

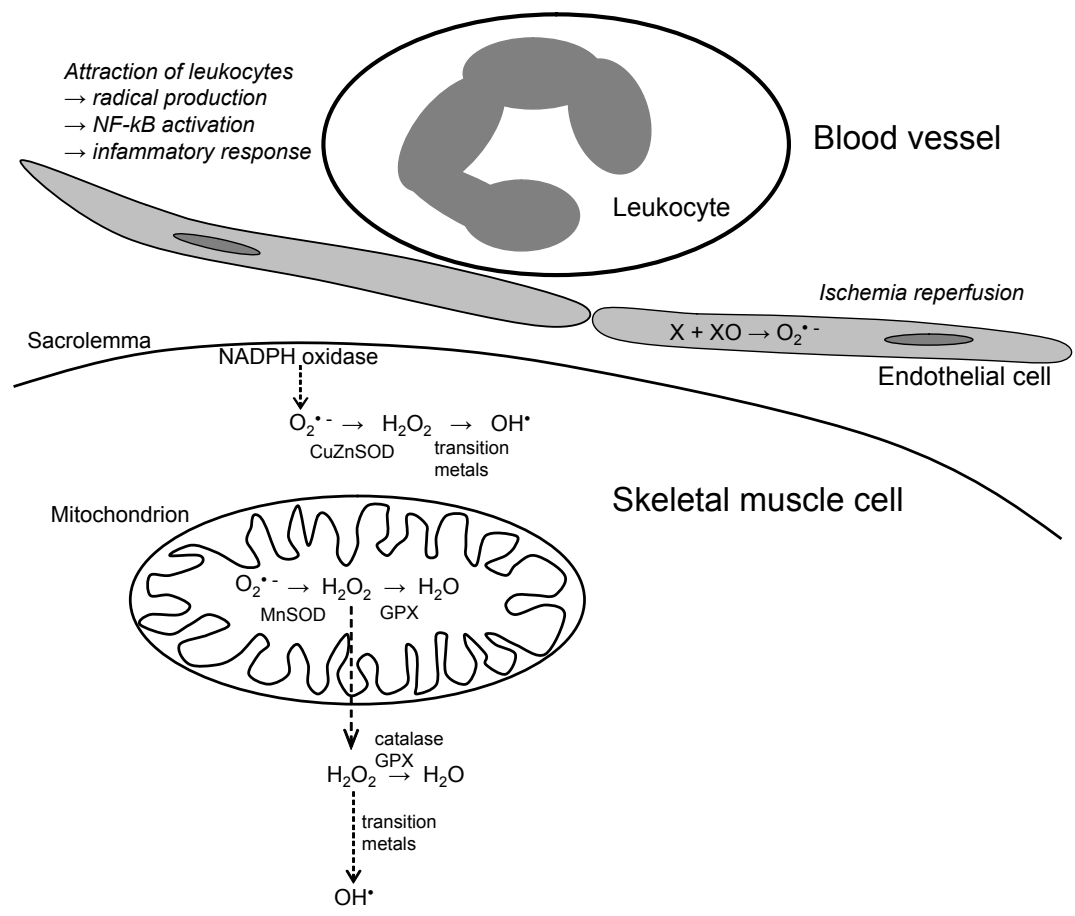

Figure 6. Important sources for ROS production during exercise in skeletal muscle. During exercise, the production of $\mathrm{O}_{2}{ }^{-}$is considerably enhanced by mitochondria and membrane NADPH oxidases. Ischemia reperfusion further increases the production of radicals. During ischemia, xanthine dehydrogenase is converted into xanthine oxidase $(\mathrm{XO})$, an enzyme producing $\mathrm{O}_{2}{ }^{\circ}$ especially during reperfusion. Moreover, ROS trigger the activation of NF- $\mathrm{kB}$ and the invasion of the injured tissue with phagocytic cells, which further promotes the production of ROS in a feed forward process.

$\mathrm{X}$ : xanthine; XO: xanthine oxidase; CuZnSOD: copper-zinc superoxide dismutase; MnSOD: manganese superoxide dismutase; GPX: glutathione peroxidase; NADPH oxidase: nicotinamide adenine dinucleotide phosphate oxidase. 
Over the past decades, progress has been made in understanding both the beneficial and detrimental roles ROS play in skeletal muscle. Low levels of ROS are required for optimal muscle contractile function (53). However, a high and unbalanced production of ROS will lead to oxidative muscle damage, for example to proteins and lipids $(18,54)$. Important targets of oxidation by ROS are PUFAs present for example in the sacrolemma, which may lead to increased cell permeability and disturbed signalling over the cell. Moreover, ROS can affect skeletal muscle force production by damaging proteins that are important in regulating cytosolic free $\mathrm{Ca}^{2+}$, such as the sarcoplasmic reticulum ryanodine receptor (RYR) and $\mathrm{Ca}^{2+}$ ATPases (55). Moreover, ROS are also able to modify an array of other regulatory proteins in skeletal muscle including myofibrillar proteins $(56,57)$, mitochondrial proteins $(58)$, and proteins that regulate glucose metabolism (59) (Figure 7).

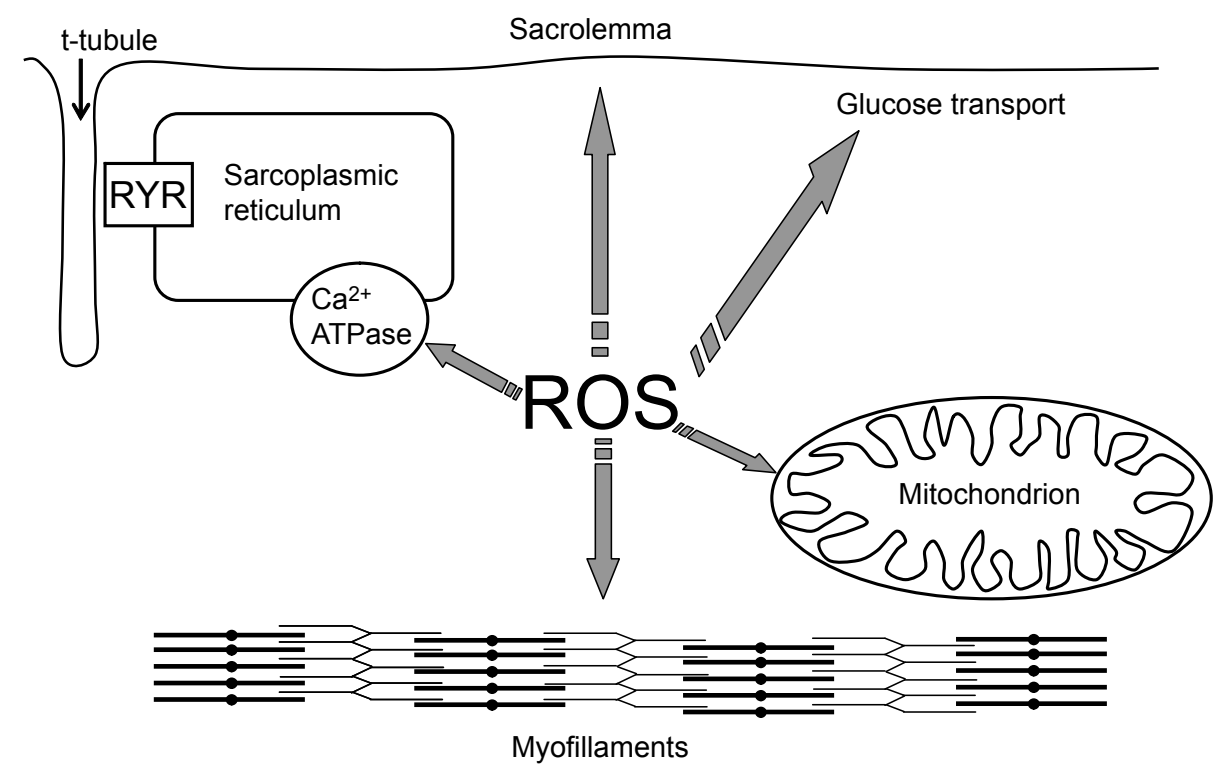

Figure 7. Targets of ROS in skeletal muscle. ROS can affect several cellular elements, such as myofillaments, mitochondria, and sarcoplasmic reticulum RYR and $\mathrm{Ca}^{2+}$ ATPases. Moreover, ROS can damage the sacrolemma and, moreover, affect glucose transport.

RYR: ryanodine receptor.

Fortunately, the body has an elaborate defence system of antioxidants that provides protection against ROS (18). In chapter 4, it was investigated whether the endogenous antioxidant defence system does provide sufficient protection against the increased radical flux induced by exercise. More specifically, the effect of exercise on oxidative damage, antioxidant capacity, and inflammation was investigated in untrained healthy volunteers. 
Oxidative damage to skeletal muscle cells might result in compromised contractile function. Increasing the antioxidant capacity of the muscle cell could provide protection against ROS generated during intense muscular contractility. This might reduce oxidative muscle damage and possibly decrease the fatigability of muscle cells. Chapter 5 describes the protective effect of hydroxytyrosol against the oxidative stress- induced impairment of force production of isolated rat skeletal muscle.

The in vivo effect of hydroxytyrosol on antioxidant capacity, oxidative damage, inflammation and lactate levels during exercise in untrained healthy volunteers is presented in chapter 6 . Finally, the results and the impact of the thesis are summarized in chapter 7 and 8 . 


\section{REFERENCES}

1. Agalias A, Magiatis P, Skaltsounis AL, et al. A new process for the management of olive oil mill waste water and recovery of natural antioxidants. J Agric Food Chem. 2007;55(7):2671-6.

2. United States Department of Agriculture. Agricultural Statistics. 2005.

3. Marangoni F, Galli C. Dietary fats of European countries in the Mediterranean area. In: Simopoulos AP, Visioli F, eds. Mediterranean diets. Vol. 87. Basel: Karger Press; 2000:78-89.

4. Boskou D. Olive oil. In: Visioli F, Simopoulos AP, eds. Mediterranean diets. Vol. 87. Basel: Karger Press; 2000:56-77.

5. Vissers MN, Zock PL, Katan MB. Bioavailability and antioxidant effects of olive oil phenols in humans: a review. Eur J Clin Nutr. 2004;58(6):955-65.

6. Owen RW, Mier W, Giacosa A, Hull WE, Spiegelhalder B, Bartsch H. Phenolic compounds and squalene in olive oils: the concentration and antioxidant potential of total phenols, simple phenols, secoiridoids, lignansand squalene. Food Chem Toxicol. 2000;38(8):647-59.

7. Mulinacci N, Romani A, Galardi C, Pinelli P, Giaccherini C, Vincieri FF. Polyphenolic content in olive oil waste waters and related olive samples. J Agric Food Chem. 2001;49(8):3509-14.

8. Khoufi S, Aloui F, Sayadi S. Extraction of antioxidants from olive mill wastewater and electrocoagulation of exhausted fraction to reduce its toxicity on anaerobic digestion. J Hazard Mater. 2008;151(2-3):531-9.

9. Fiorentino A, Gentili A, Isidori $M$, et al. Environmental effects caused by olive mill wastewaters: toxicity comparison of low-molecular-weight phenol components. J Agric Food Chem. 2003;51(4):1005-9.

10. Keys A, Menotti A, Karvonen MJ, et al. The diet and 15-year death rate in the seven countries study. Am J Epidemiol. 1986;124(6):903-15.

11. Hu FB. The Mediterranean diet and mortality--olive oil and beyond. $\mathrm{N}$ Engl J Med. 2003;348(26):2595-6.

12. Covas MI. Olive oil and the cardiovascular system. Pharmacol Res. 2007;55(3):175-86.

13. Covas MI, Nyyssonen K, Poulsen HE, et al. The effect of polyphenols in olive oil on heart disease risk factors: a randomized trial. Ann Intern Med. 2006;145(5):333-41.

14. Fito $M$, Cladellas $M$, de la Torre $R$, et al. Antioxidant effect of virgin olive oil in patients with stable coronary heart disease: a randomized, crossover, controlled, clinical trial. Atherosclerosis. 2005;181(1):149-58.

15. Marrugat J, Covas MI, Fito M, et al. Effects of differing phenolic content in dietary olive oils on lipids and LDL oxidation--a randomized controlled trial. Eur J Nutr. 2004;43(3):140-7.

16. Weinbrenner $\mathrm{T}$, Fito $\mathrm{M}$, de la Torre $\mathrm{R}$, et al. Olive oils high in phenolic compounds modulate oxidative/antioxidative status in men. J Nutr. 2004;134(9):2314-21.

17. Halliwell B, Chirico S. Lipid peroxidation: its mechanism, measurement, and significance. Am J Clin Nutr. 1993;57(5 Suppl):715S-724S; discussion 724S-725S.

18. Bast A, Haenen GR, Doelman CJ. Oxidants and antioxidants: state of the art. Am J Med. 1991;91(3C):2S-13S.

19. Madamanchi NR, Hakim ZS, Runge MS. Oxidative stress in atherogenesis and arterial thrombosis: the disconnect between cellular studies and clinical outcomes. J Thromb Haemost. 2005;3(2):254-67.

20. Boots AW, Haenen GR, Bast A. Oxidant metabolism in chronic obstructive pulmonary disease. Eur Respir J Suppl. 2003;46:14s-27s.

21. Deiana M, Aruoma Ol, Bianchi ML, et al. Inhibition of peroxynitrite dependent DNA base modification and tyrosine nitration by the extra virgin olive oil-derived antioxidant hydroxytyrosol. Free Radic Biol Med. 1999;26(5-6):762-9.

22. de la Puerta R, Martinez Dominguez ME, Ruiz Gutierrez V, Flavill JA, Hoult JR. Effects of virgin olive oil phenolics on scavenging of reactive nitrogen species and upon nitrergic neurotransmission. Life Sci. 2001;69(10):1213-22. 
23. Visioli F, Bellomo G, Galli C. Free radical-scavenging properties of olive oil polyphenols. Biochem Biophys Res Commun. 1998;247(1):60-4.

24. O'Dowd Y, Driss F, Dang PM, et al. Antioxidant effect of hydroxytyrosol, a polyphenol from olive oil: scavenging of hydrogen peroxide but not superoxide anion produced by human neutrophils. Biochem Pharmacol. 2004;68(10):2003-8.

25. Manna C, Galletti P, Cucciolla V, Moltedo O, Leone A, Zappia V. The protective effect of the olive oil polyphenol (3,4-dihydroxyphenyl)-ethanol counteracts reactive oxygen metaboliteinduced cytotoxicity in Caco-2 cells. J Nutr. 1997;127(2):286-92.

26. Manna C, Galletti P, Cucciolla V, Montedoro G, Zappia V. Olive oil hydroxytyrosol protects human erythrocytes against oxidative damages. J Nutr Biochem. 1999;10:159-165.

27. Goya L, Mateos R, Bravo L. Effect of the olive oil phenol hydroxytyrosol on human hepatoma HepG2 cells : Protection against oxidative stress induced by tert-butylhydroperoxide. Eur J Nutr. 2007.

28. Gutierrez VR, de la Puerta R, Catala A. The effect of tyrosol, hydroxytyrosol and oleuropein on the non-enzymatic lipid peroxidation of rat liver microsomes. Mol Cell Biochem. 2001;217(12):35-41.

29. Fito M, Covas MI, Lamuela Raventos RM, et al. Protective effect of olive oil and its phenolic compounds against low density lipoprotein oxidation. Lipids. 2000;35(6):633-8.

30. Visioli F, Bellomo G, Montedoro G, Galli C. Low density lipoprotein oxidation is inhibited in vitro by olive oil constituents. Atherosclerosis. 1995;117(1):25-32.

31. Hansson GK. Inflammation, atherosclerosis, and coronary artery disease. $\mathrm{N}$ Engl J Med. 2005;352(16):1685-95.

32. Tuck KL, Freeman MP, Hayball PJ, Stretch GL, Stupans I. The in vivo fate of hydroxytyrosol and tyrosol, antioxidant phenolic constituents of olive oil, after intravenous and oral dosing of labeled compounds to rats. J Nutr. 2001;131(7):1993-6.

33. Visioli F, Caruso D, Plasmati E, et al. Hydroxytyrosol, as a component of olive mill waste water, is dose- dependently absorbed and increases the antioxidant capacity of rat plasma. Free Radic Res. 2001;34(3):301-5.

34. Visioli F, Galli C, Bornet F, et al. Olive oil phenolics are dose-dependently absorbed in humans. FEBS Lett. 2000;468(2-3):159-60.

35. Vissers MN, Zock PL, Roodenburg AJ, Leenen R, Katan MB. Olive oil phenols are absorbed in humans. J Nutr. 2002;132(3):409-17.

36. Miro Casas E, Covas MI, Farre M, et al. Hydroxytyrosol disposition in humans. Clin Chem. 2003;49(6 Pt 1):945-52.

37. Corona G, Tzounis X, Assunta Dessi M, et al. The fate of olive oil polyphenols in the gastrointestinal tract: implications of gastric and colonic microflora-dependent biotransformation. Free Radic Res. 2006;40(6):647-58.

38. Edgecombe SC, Stretch GL, Hayball PJ. Oleuropein, an antioxidant polyphenol from olive oil, is poorly absorbed from isolated perfused rat intestine. J Nutr. 2000;130(12):2996-3002.

39. Visioli F, Galli C, Plasmati E, et al. Olive phenol hydroxytyrosol prevents passive smokinginduced oxidative stress. Circulation. 2000;102(18):2169-71.

40. Visioli F, Caruso D, Galli C, Viappiani S, Galli G, Sala A. Olive oils rich in natural catecholic phenols decrease isoprostane excretion in humans. Biochem Biophys Res Commun. 2000;278(3):797-9.

41. Covas MI, de la Torre K, Farre-Albaladejo M, et al. Postprandial LDL phenolic content and LDL oxidation are modulated by olive oil phenolic compounds in humans. Free Radic Biol Med. 2006;40(4):608-16.

42. Bonanome A, Pagnan A, Caruso D, et al. Evidence of postprandial absorption of olive oil phenols in humans. Nutr Metab Cardiovasc Dis. 2000;10(3):111-20.

43. Nicolaiew N, Lemort N, Adorni L, et al. Comparison between extra virgin olive oil and oleic acid rich sunflower oil: effects on postprandial lipemia and LDL susceptibility to oxidation. Ann Nutr Metab. 1998;42(5):251-60. 


\section{CHAPTER 1}

44. Vissers MN, Zock PL, Leenen R, Roodenburg AJ, van Putte KP, Katan MB. Effect of consumption of phenols from olives and extra virgin olive oil on LDL oxidizability in healthy humans. Free Radic Res. 2001;35(5):619-29.

45. Vissers MN, Zock PL, Wiseman SA, Meyboom S, Katan MB. Effect of phenol-rich extra virgin olive oil on markers of oxidation in healthy volunteers. Eur J Clin Nutr. 2001;55(5):334-41.

46. Visioli F, Galli C. Oleuropein protects low density lipoprotein from oxidation. Life Sci. 1994;55(24):1965-71.

47. Ross R. Atherosclerosis--an inflammatory disease. N Engl J Med. 1999;340(2):115-26.

48. Kinlay S, Ganz P. Relation between endothelial dysfunction and the acute coronary syndrome: implications for therapy. Am J Cardiol. 2000;86(8B):10J-13J; discussion 13J-14J.

49. Sjodin B, Hellsten Westing Y, Apple FS. Biochemical mechanisms for oxygen free radical formation during exercise. Sports Med. 1990;10(4):236-54.

50. Reid MB. Invited Review: redox modulation of skeletal muscle contraction: what we know and what we don't. J Appl Physiol. 2001;90(2):724-31.

51. Finaud J, Lac G, Filaire E. Oxidative stress : relationship with exercise and training. Sports Med. 2006;36(4):327-58.

52. Jackson MJ, Pye D, Palomero J. The production of reactive oxygen and nitrogen species by skeletal muscle. J Appl Physiol. 2007;102(4):1664-1670.

53. Reid MB, Khawli FA, Moody MR. Reactive oxygen in skeletal muscle. III. Contractility of unfatigued muscle. J Appl Physiol. 1993;75(3):1081-7.

54. Vollaard NB, Shearman JP, Cooper CE. Exercise-induced oxidative stress:myths, realities and physiological relevance. Sports Med. 2005;35(12):1045-62.

55. Zissimopoulos S, Lai FA. Redox regulation of the ryanodine receptor/calcium release channel. Biochem Soc Trans. 2006;34(Pt 5):919-21.

56. Nagasawa T, Hatayama T, Watanabe $Y$, Tanaka M, Niisato $Y$, Kitts DD. Free radical-mediated effects on skeletal muscle protein in rats treated with Fe-nitrilotriacetate. Biochem Biophys Res Commun. 1997;231(1):37-41.

57. Coirault C, Guellich A, Barbry T, Samuel JL, Riou B, Lecarpentier Y. Oxidative stress of myosin contributes to skeletal muscle dysfunction in rats with chronic heart failure. Am J Physiol Heart Circ Physiol. 2007;292(2):H1009-17.

58. Kim NH, Jeong MS, Choi SY, Kang JH. Oxidative modification of cytochrome c by hydrogen peroxide. Mol Cells. 2006;22(2):220-7.

59. Balon TW, Yerneni KK. Redox regulation of skeletal muscle glucose transport. Med Sci Sports Exerc. 2001;33(3):382-5. 


\section{CHAPTER 2}

New insights into controversies on the antioxidant potential of the olive oil antioxidant hydroxytyrosol

Saskia J. Rietjens, Aalt Bast, and Guido R.M.M. Haenen

J Agric Food Chem 2007;55(18):7609-14

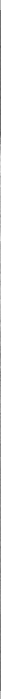




\section{ABSTRACT}

In the present study, the antioxidant profile of olive oil antioxidants was investigated. Hydroxytyrosol and oleuropein are potent scavengers of hydroxyl radicals $\left(\mathrm{OH}^{*}\right)$, peroxynitrite $(\mathrm{ONOOH})$, and superoxide radicals $\left(\mathrm{O}_{2}{ }^{*}\right)$. Homovanillic alcohol, one of the main metabolites of hydroxytyrosol, and tyrosol are less potent scavengers of these reactive species. None of the olive oil antioxidants are good hypochlorous acid $(\mathrm{HOCl})$ or hydrogen peroxide $\left(\mathrm{H}_{2} \mathrm{O}_{2}\right)$ scavengers.

Hydroxytyrosol efficiently protects against low density lipoprotein (LDL) oxidation in vitro and in vivo. However, no protective effect of hydroxytyrosol is usually demonstrated ex vivo against the oxidation of LDL isolated from humans after hydroxytyrosol consumption. The present study shows that this controversy is due to the isolation of $\mathrm{LDL}$, which greatly reduces the protective effect of hydroxytyrosol against LDL oxidation. Hydroxytyrosol is an efficient scavenger of several free radicals. The physiological relevance of the high intrinsic antioxidant activity of hydroxytyrosol is illustrated by its protection against LDL oxidation. 


\section{INTRODUCTION}

The role of olive oil in the protection against cardiovascular disease has been highlighted in several studies (1-5). Olive oil, the main source of fat of the Mediterranean diet, is rich in oleic acid, a mono-unsaturated fatty acid (MUFA). To date, most of the research on the protective effect of olive oil has been focused on its high MUFA content.

Recent studies show that olive oil is more than just a mono-unsaturated fat. Olive oil is a natural product that contains a wide range of ingredients. The composition depends, among others, on the type of olives, the manufacturing process, and the storage conditions. In general, extra virgin olive oil contains relatively high amounts of phenolic antioxidants (6), such as oleuropein, hydroxytyrosol, and tyrosol (Figure 1). These phenolic compounds have been shown to provide beneficial cardiovascular effects $(1,2,7,8)$. Consequently, supplements containing phenolic antioxidants from olive oil with healthpromoting claims have been developed.<smiles>OCCc1ccc(O)c(O)c1</smiles>

hydroxytyrosol<smiles>OCCc1ccc(O)cc1</smiles>

tyrosol<smiles>CC=C1C(OC(C)C)OC=C(C(=O)OC)C1CC(=O)OCCc1ccc(O)c(O)c1</smiles>

oleuropein<smiles>COc1cc(CCO)ccc1O</smiles>

homovanillic alcohol

Figure 1. Molecular structure of hydroxytyrosol, oleuropein, tyrosol and homovanillic alcohol.

The molecular mechanism of the cardiovascular effects of olive oil antioxidants has not been fully elucidated. Studies investigating the antioxidant effects of olive oil phenolic compounds are conflicting (9). It has been shown in both in vitro and human in vivo studies that phenolic compounds efficiently protect against LDL oxidation (1, 2, 7, 8, 10-13). However, no protective effect of hydroxytyrosol is usually found against LDL oxidation in ex vivo experiments (14-17). In these experiments, LDL is isolated from humans after hydroxytyrosol consumption, and subsequently the oxidizability of this LDL is determined ex vivo. 
The present study is designed to provide new insights into the controversy on the effect of phenolic compounds on LDL oxidation. Some controversies also exist regarding the antioxidant profile of phenolic compounds present in olive oil. For example, the reports on radical scavenging are ambiguous $(4,18)$. Therefore, an integral antioxidant profile of hydroxytyrosol and related compounds is determined by quantifying their scavenging potential of $\mathrm{OH}^{\circ}$, $\mathrm{ONOOH}, \mathrm{O}_{2}{ }^{-}, \mathrm{HOCl}$ and $\mathrm{H}_{2} \mathrm{O}_{2}$. The intrinsic antioxidant activity forms the fundament for the physiological impact of olive oil phenolic compounds.

\section{MATERIALS AND METHODS}

\section{Chemicals}

Hydroxytyrosol was obtained from Cayman Chemical, Ann Arbor, MI, USA. Oleuropein was obtained from Extrasynthese, Lyon, France. Tyrosol was purchased from Fluka, Buchs, Switzerland. Dihydrorhodamine-123 (DHR-123), 2-deoxy-D-ribose, homovanillic alcohol, ascorbate, rutin, 2-methoxyphenol, phenol, $\mathrm{HOCl}, \mathrm{H}_{2} \mathrm{O}_{2}$, dithionitrobenzoic acid, and mannitol were purchased from Sigma, St. Louis, MO, USA. Catechol was obtained from Janssen Chimica, Geel, Belgium. Lipoic acid was purchased from Asta Medica AG, Frankfurt, Germany. Reduced glutathione (GSH) was obtained from ICN Biomedicals Inc., Costa Mesa, CA, USA. Ebselen was obtained from Rhône-Poulenc Rorer, Köln, Germany. All other chemicals were of analytical grade purity.

\section{Scavenging of reactive oxygen and nitrogen species}

The antioxidant profile of hydroxytyrosol, oleuropein, tyrosol, and homovanillic alcohol was determined by quantifying their scavenging potential of reactive oxygen and nitrogen species. Scavenging of $\mathrm{OH}^{*}$ was determined by the deoxyribose method according to Halliwell et al. (19). Scavenging of $\mathrm{O}_{2}{ }^{-}$was measured according to den Hartog et al. (20). The protection against $\mathrm{ONOOH}$ induced DHR-123 oxidation was measured as described by Kooy et al. (21). The $\mathrm{HOCl}$ scavenging potential of the compounds was measured according to Ching et al. (22). Scavenging of $\mathrm{H}_{2} \mathrm{O}_{2}$ was measured by adding $1 \mathrm{mM} \mathrm{H}_{2} \mathrm{O}_{2}$ to a $100 \mu \mathrm{M}$ solution of the scavenger in a $150 \mathrm{mM}$ sodium phosphate buffer $(\mathrm{pH}$ $7.4,37^{\circ} \mathrm{C}$ ). UV spectra were recorded before and during $30 \mathrm{~min}$ after the addition of $\mathrm{H}_{2} \mathrm{O}_{2}$.

The reactive oxygen and nitrogen species scavenging activities of the compounds tested were related to reference antioxidants that either have a high scavenging activity for one of these reactive species or were used for this purpose before. The reference compounds for $\mathrm{OH}^{\circ}, \mathrm{ONOOH}, \mathrm{O}_{2}{ }^{-}, \mathrm{HOCl}$, and 
$\mathrm{H}_{2} \mathrm{O}_{2}$ scavenging were, respectively, mannitol (23), ebselen (24), rutin, lipoic acid (25), and GSH.

\section{LDL isolation}

Blood was collected from a healthy volunteer in tubes containing heparin and centrifuged at $3500 \mathrm{rpm}$ at $4{ }^{\circ} \mathrm{C}$ for $10 \mathrm{~min}$ to obtain plasma. This study was performed in compliance with the guidelines of the Medical Ethical Review Board of the Academic Hospital, Maastricht, The Netherlands. Potassium bromide was added to plasma to increase its density (0.325 $\mathrm{g}$ per $\mathrm{ml}$ plasma). $\mathrm{LDL}$ was isolated by density gradient ultracentrifugation at $32,000 \mathrm{rpm}$ at $4{ }^{\circ} \mathrm{C}$ for $17 \mathrm{~h}$. The LDL fraction was isolated in a density range of $1.019-1.063 \mathrm{~g}$ $\mathrm{NaCl} / \mathrm{ml}$ and diluted to a concentration of $1 \mathrm{mg} / \mathrm{ml}$ in a $50 \mathrm{mM}$ sodium phosphate buffer at $\mathrm{pH} 7.4$.

\section{LDL oxidation}

$\mathrm{LDL}$ oxidation was performed at $37^{\circ} \mathrm{C}$ in a $50 \mathrm{mM}$ sodium phosphate buffer at $\mathrm{pH} 7.4$ by adding $50 \mu \mathrm{M} \mathrm{CuSO}_{4}$ to $50 \mu \mathrm{g} / \mathrm{ml} \mathrm{LDL}$ in the presence of hydroxytyrosol or vehicle (ethanol). Conjugated diene formation was measured at 234 nm during $240 \mathrm{~min}$.

The effect of the LDL isolation procedure on the protection by hydroxytyrosol against LDL oxidation was also determined. Hydroxytyrosol or vehicle (ethanol) was added to isolated LDL $(1 \mathrm{mg} / \mathrm{ml})$, vortexed, and incubated for $5 \mathrm{~min}$. Subsequently, the LDL isolation procedure was repeated by creating the same density gradient. After ultracentrifugation, LDL was isolated and subsequently oxidized as described above. Mean lag times were calculated for all of the LDL experiments.

The addition of hydroxytyrosol (final concentration of $5 \mu \mathrm{M}$ ) to LDL that was isolated for a second time gave the same protection (data not shown) as hydroxytyrosol $(5 \mu \mathrm{M})$ added to $\mathrm{LDL}$ after the first isolation procedure. This indicates that, with respect to the ability of hydroxytyrosol to protect, LDL has not been affected by the isolation procedure.

\section{Lipophilicity}

The lipophilicity of hydroxytyrosol was measured by determining the logarithm of the octanol water partitioning coefficient $\left(\log \mathrm{P}_{\mathrm{o} / \mathrm{w}}\right)$. The octanol water partitioning coefficient $\left(\mathrm{P}_{\mathrm{o} / \mathrm{w}}\right)$ of hydroxytyrosol was calculated by dividing the concentration of hydroxytyrosol in the octanol layer by that in the aqueous layer (150 mM sodium phosphate buffer, $\mathrm{pH}$ 7.4) after mixing a $1 \mathrm{mM}$ solution of hydroxytyrosol in buffer that was saturated with octanol with an equal 
volume of octanol that was saturated with buffer. The concentration of hydroxytyrosol in each layer was determined spectrophotometrically at $283 \mathrm{~nm}$.

\section{Statistics}

The results are expressed as means \pm SEM $(n=3)$. The LDL oxidation results are based on at least two separate experiments. For the results based on two experiments, the lag time is presented as mean \pm half-range.

\section{RESULTS}

Both oleuropein and hydroxytyrosol were potent $\mathrm{OH}^{\circ}, \mathrm{O}_{2}{ }^{*}$, and $\mathrm{ONOOH}$ scavengers (Figure 2). Homovanillic alcohol, one of the main metabolites of hydroxytyrosol (Figure 1), was a relatively good scavenger of $\mathrm{ONOOH}$ and $\mathrm{OH}^{*}$. However, homovanillic alcohol was a less potent scavenger of $\mathrm{OH}^{*}, \mathrm{O}_{2}{ }^{-}$ and $\mathrm{ONOOH}$ than hydroxytyrosol. Compared to hydroxytyrosol, tyrosol was also a relatively poor scavenger of $\mathrm{OH}^{\circ}, \mathrm{O}_{2}{ }^{\circ}$, and $\mathrm{ONOOH}$. None of the phenolic compounds from olive oil were good $\mathrm{HOCl}$ or $\mathrm{H}_{2} \mathrm{O}_{2}$ scavengers. The $\mathrm{HOCl}$ and $\mathrm{H}_{2} \mathrm{O}_{2}$ scavenging activity of the reference compounds was at least 10 to 100 times higher than that of the phenolic compounds.

To identify the functional group that is important in the antioxidant potential of the compounds studied, the scavenging activities of catechol, 2methoxyphenol, and phenol were also determined (Figure 2). Catechol was a potent scavenger of $\mathrm{OH}^{*}, \mathrm{O}_{2}{ }^{-}$, and $\mathrm{ONOOH}$. Phenol was less potent in scavenging $\mathrm{OH}^{\circ}, \mathrm{O}_{2}{ }^{\circ}$, and $\mathrm{ONOOH}$ compared to catechol. The compound 2methoxyphenol was a relatively good scavenger of $\mathrm{OH}^{\circ}, \mathrm{O}_{2}{ }^{\circ}$, and $\mathrm{ONOOH}$, although the scavenging activities were lower than that of catechol. Catechol, 2-methoxyphenol, and phenol did not display any relevant $\mathrm{H}_{2} \mathrm{O}_{2}$ or $\mathrm{HOCl}$ scavenging activity.

Hydroxytyrosol dose dependently protected against LDL oxidation. A representative experiment is shown in Figure 3. When $5 \mu \mathrm{M}$ hydroxytyrosol was added to $\mathrm{LDL}$, the lag time in the in vitro assay was more than $240 \mathrm{~min}$, whereas the lag time of the control experiment was 30 min (Figure 3A).

To examine the effect of the isolation of $L D L$, the isolation procedure was performed for a second time. Hydroxytyrosol $(5 \mu \mathrm{M})$ was added to isolated $\mathrm{LDL}$, and subsequently, the LDL isolation procedure was repeated for a second time. The protective effect of hydroxytyrosol against LDL oxidation was drastically reduced by the second isolation procedure. The lag time of oxidation was shortened from more than 240 min before the second LDL isolation procedure to $60 \mathrm{~min}$ after the second LDL isolation procedure (Figure 3B). 

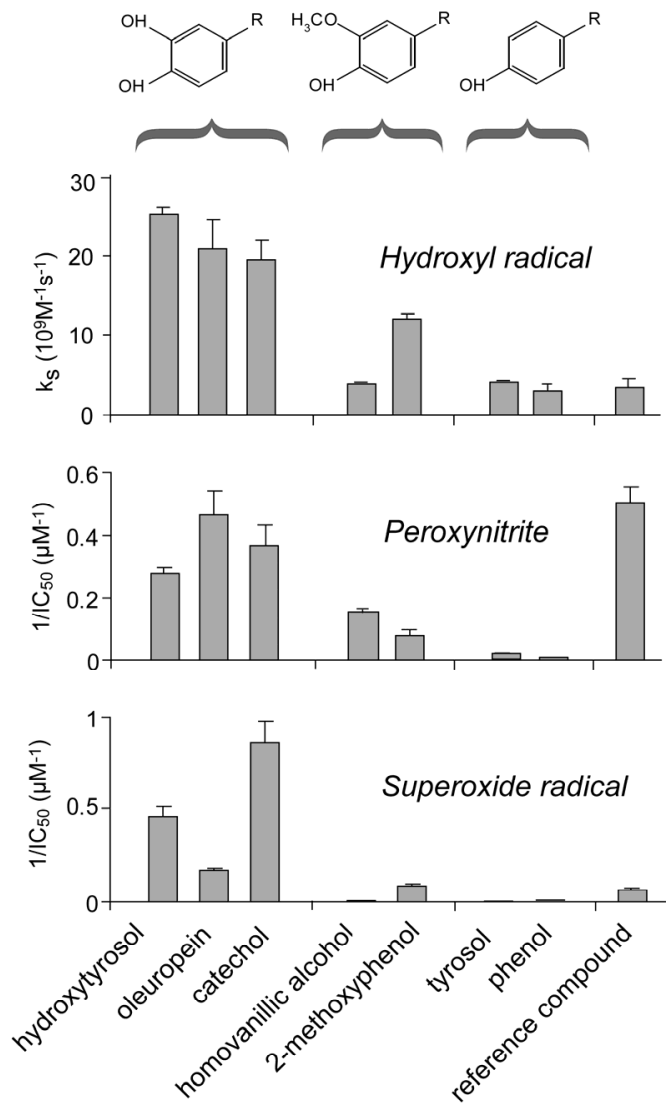

Figure 2. Antioxidant profiles of hydroxytyrosol, oleuropein, homovanillic alcohol, tyrosol, and related compounds. The $\mathrm{OH}^{*}, \mathrm{ONOOH}$, and $\mathrm{O}_{2}{ }^{-}$scavenging activities of the compounds were related to the scavenging activities of respectively mannitol (second-order rate constant $=3.4 \cdot 10^{9}$ $\left.\mathrm{M}^{-1} \cdot \mathrm{s}^{-1}\right)$, ebselen $\left(\mathrm{IC}_{50}=2.0 \mu \mathrm{M}\right)$, and rutin $\left(\mathrm{IC}_{50}=14.8 \mu \mathrm{M}\right)$. The $\mathrm{H}_{2} \mathrm{O}_{2}$ and $\mathrm{HOCl}$ scavenging activity of the compounds was related to $\mathrm{GSH}\left(\mathrm{IC}_{50}=83 \mu \mathrm{M}\right)$ and lipoic acid (second order rate constant $=0.1 \mathrm{mM}^{-1} \cdot \mathrm{min}^{-1}$ ), respectively. None of the phenolic compounds displayed any relevant $\mathrm{H}_{2} \mathrm{O}_{2}$ or $\mathrm{HOCl}$ scavenging activity. The $\mathrm{H}_{2} \mathrm{O}_{2}$ and $\mathrm{HOCl}$ scavenging activity of the reference compounds was at least 10 to 100 times higher than that of the phenolic compounds.

The lipophilicity of hydroxytyrosol, which was measured by determining the $\log P_{o / w}$ value, was found to be 0.04 . This is similar to the $P_{o / w}$ value reported previously (26). This means that the concentration of hydroxytyrosol in biological systems is expected to be very similar in aqueous and lipid compartments. 

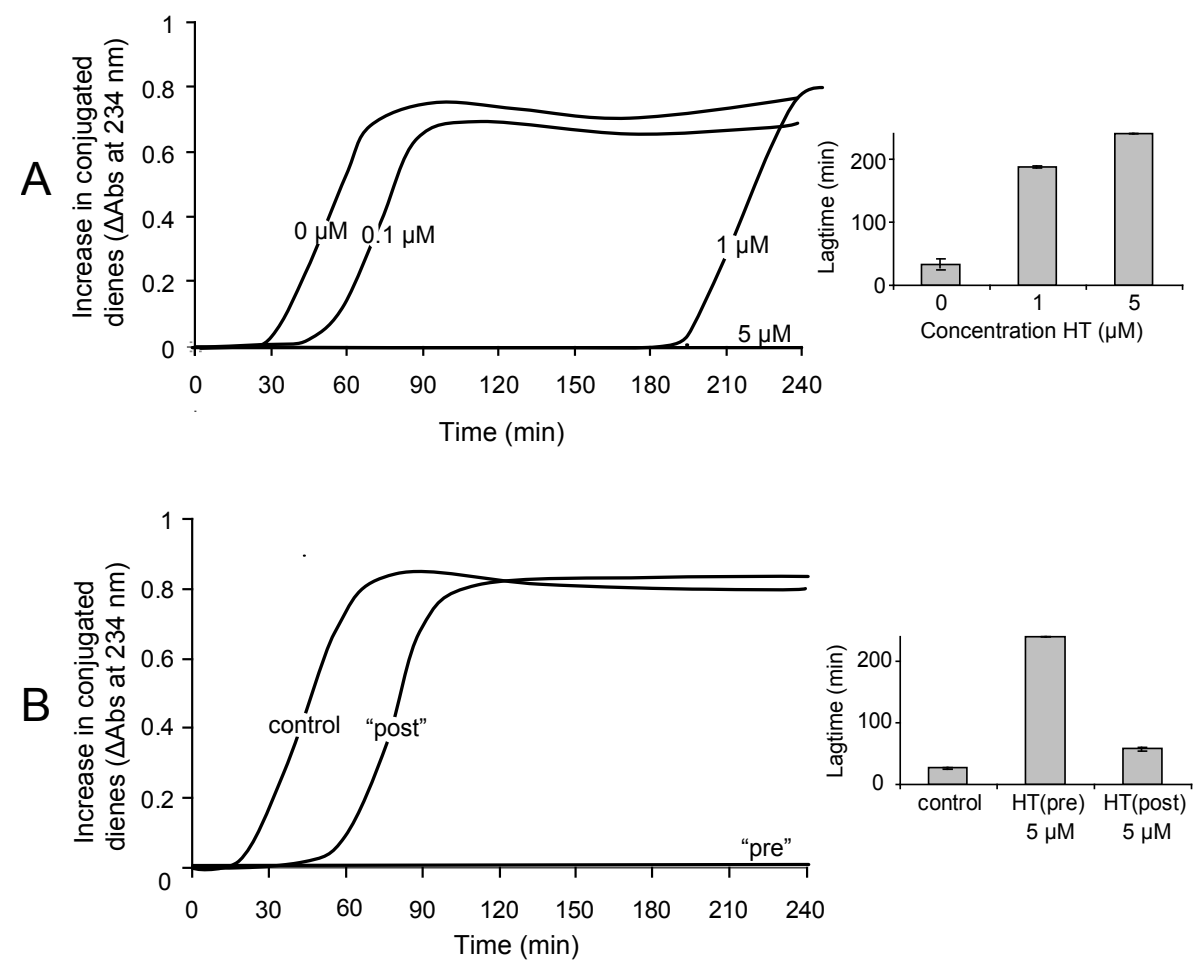

Figure 3. Protection by hydroxytyrosol (HT) against LDL oxidation. In panel A, the dose-dependent protection by hydroxytyrosol against LDL oxidation is shown. In panel B, the effect of the LDL isolation procedure on the protection by hydroxytyrosol against LDL oxidation is shown. Hydroxytyrosol is added to isolated LDL, and subsequently, the LDL isolation procedure is repeated. The protective effect of hydroxytyrosol before and after the second LDL isolation procedure is shown. The lag times of LDL oxidation are shown in the inserts. No conjugated diene formation occurred when a concentration of $5 \mu \mathrm{M}$ hydroxytyrosol was used (before the second isolation procedure; panels A and B). For this reason, the lag time was set at $240 \mathrm{~min}$, that is, the duration of the experiment.

\section{DISCUSSION}

The aim of the present study was to investigate the antioxidant potential of olive oil phenolic compounds. The fundament of the beneficial effect of an antioxidant is its intrinsic antioxidant activity. Therefore, the antioxidant profile of the olive oil antioxidants was established. Subsequently, the physiological impact of the antioxidant efficacy was evaluated by determining the protective effect against $L D L$ oxidation.

In the present study, it was shown that both hydroxytyrosol and oleuropein have superior $\mathrm{ONOOH}, \mathrm{OH}^{*}$, and $\mathrm{O}_{2}{ }^{-}$scavenging activities, equal to or 
surpassing that of the reference antioxidants used. The high potency of hydroxytyrosol and oleuropein for scavenging $\mathrm{ONOOH}$ observed in the present study is in accordance with previous reports $(27,28)$. Literature on the activity of hydroxytyrosol as an $\mathrm{O}_{2}^{-{ }^{-}}$scavenger is contradictory $(4,18)$. Our results indicate that hydroxytyrosol and oleuropein do scavenge $\mathrm{O}_{2}{ }^{\circ}$.

In the present study, it was shown that none of the olive oil phenolic compounds were good $\mathrm{H}_{2} \mathrm{O}_{2}$ or $\mathrm{HOCl}$ scavengers. Previous reports have suggested that hydroxytyrosol is able to scavenge $\mathrm{H}_{2} \mathrm{O}_{2}$ (18) and $\mathrm{HOCl}$ (4). The reported scavenging of $\mathrm{H}_{2} \mathrm{O}_{2}$ (18) by hydroxytyrosol is most likely the result of the scavenging of reactive species that are formed out of $\mathrm{H}_{2} \mathrm{O}_{2}$. In the only report on the $\mathrm{HOCl}$ scavenging of hydroxytyrosol, the activity of hydroxytyrosol was related to poor $\mathrm{HOCl}$ scavengers, that is, vitamin $\mathrm{C}$ and vitamin $\mathrm{E}$. Comparing the activity of hydroxytyrosol to a more relevant reference compound clearly shows that hydroxytyrosol hardly possesses any $\mathrm{HOCl}$ scavenging activity. The scavenging of the non-radical species $\mathrm{H}_{2} \mathrm{O}_{2}$ and $\mathrm{HOCl}$ involves a two-electron reaction. In the scavenging of these species, thiols appear to be superior to phenolic compounds.

Our results show that compounds having a catechol (orthodiphenolic) group in their molecular structure, that is, oleuropein and hydroxytyrosol, have superior antioxidant properties than compounds lacking this moiety, that is, homovanillic alcohol and tyrosol. As also reported previously, the present study shows that the antioxidant potency of the catechol group is superior to that of a resorcinol or phenol group. The relatively high antioxidant activity of catechol can be explained by the high electron donating effect of the second hydroxyl group $(29,30)$.

For an antioxidant to have beneficial health effects in vivo, it is of course essential that it is taken up. Several studies in humans and rats indeed report that the uptake of hydroxytyrosol is good (31-34). Plasma concentrations of hydroxytyrosol after consumption of $25 \mathrm{ml}$ of extra virgin olive oil range from 50 to $160 \mathrm{nM}(8,35)$. The consumption of supplements containing a relatively high amount of hydroxytyrosol might lead to an even higher hydroxytyrosol concentration. Hydroxytyrosol is metabolized in the body by the action of catechol-O-methyltransferase to homovanillic alcohol. Oleuropein itself hardly reaches the systemic circulation after ingestion. However, most of the oleuropein is hydrolyzed in the intestine, yielding hydroxytyrosol $(36,37)$.

The physiological impact of the antioxidant efficacy of hydroxytyrosol was evaluated by determining its protective effect against LDL oxidation. LDL oxidation is one of the key steps in the initiation of atherosclerosis. The present study shows that hydroxytyrosol efficiently protects against LDL oxidation in vitro at relatively low concentrations. Review of the literature shows that our results are consistent with in vitro data from other studies on human LDL (Table 1). The present study also shows that the isolation procedure substantially 
reduces the protective effect of hydroxytyrosol against LDL oxidation. This might explain the false negative results in ex vivo studies that usually show no protective effect of olive oil phenolic compounds in LDL isolated from humans having consumed a diet rich in olive oil (Table 1).

During the isolation procedure, a relatively small volume of LDL is added to a relatively large volume of sodium chloride solutions. In the present study, it was shown that the $\log \mathrm{P}_{\mathrm{o} / \mathrm{w}}$ of hydroxytyrosol is 0.04 . This indicates that the reduction in the protective effect might be due to the loss of hydroxytyrosol from LDL during the LDL isolation procedure. Recently, the loss of hydroxytyrosol from LDL during the isolation procedure has also been addressed by de la Torre-Carbot et al. (38). This is in line with the results of our study. The loss of antioxidants other than hydroxytyrosol during the isolation procedure might also be involved in the underestimation of the protective effect of hydroxytyrosol in ex vivo experiments. In addition to the lipophilicity, also other properties determine the interaction of antioxidants with LDL, such as hydrogen binding to proteins. Possible structural LDL changes during the isolation procedure may affect this interaction, which can also result in an underestimation of the protective effect of hydroxytyrosol in ex vivo experiments.

The ex vivo protective effect of olive oil phenolic compounds reported by Ramirez-Tortosa et al. (39) might be due to the higher vitamin E content in the olive oil diet compared to the control diet. Vitamin E, an extremely lipophilic compound, will not be removed from LDL during the isolation procedure. Marrugat et al. also demonstrated a protective effect of olive oil phenolic compounds against LDL oxidation, measured ex vivo after hydroxytyrosol consumption (2). However, the observed increase in lag time of LDL oxidation was only marginal.

LDL oxidizability has also been evaluated in vivo in several studies (Table 1). Both long and short term ingestion of olive oil rich in phenolic compounds have been shown to lower plasma levels of oxidized $\operatorname{LDL}(1,2,7,8)$. These effects are not attributable to the mono-unsaturated fatty acids of olive oil, as the fat compositions of the olive oil and the control oil were identical. These studies unequivocally proof that olive oil phenolic compounds provide protection against $L D L$ oxidation.

In conclusion, hydroxytyrosol is an efficient scavenger of several free radicals. The physiological relevance of the high intrinsic antioxidant activity of hydroxytyrosol is illustrated by its protection against LDL oxidation.

\section{Acknowledgements}

We would like to thank Marie-José Drittij, Marc Fischer, and Mathijs Groeneweg for their valuable assistance. 
Table 1. Overview of human studies on the protective effect of olive oil phenolic compounds against LDL oxidation

\begin{tabular}{|c|c|c|c|}
\hline IN VITRC & & & \\
\hline Concentr & oxytyrosol $(\mu \mathrm{M})$ & Effect & Reference \\
\hline $\begin{array}{l}0.1 \\
1 \\
5\end{array}$ & & $\begin{array}{l}+++ \\
++++ \\
++++\end{array}$ & Present study \\
\hline $\begin{array}{l}0.3^{\mathrm{a}, \mathrm{b}} \\
1.1^{\mathrm{a}, \mathrm{b}} \\
1.7^{\mathrm{a}, \mathrm{b}}\end{array}$ & & $\begin{array}{l}++ \\
+++ \\
++++\end{array}$ & 12 \\
\hline $10^{b}$ & & +++ & 10 \\
\hline $10^{b, c}$ & & ++++ & 11 \\
\hline EX VIVO & & & \\
\hline $\begin{array}{l}\text { Estimated } \\
\text { (mg phen }\end{array}$ & $\begin{array}{l}\text { ke } \\
\text { alents) }\end{array}$ & & \\
\hline Olive oil & Control & Effect & Reference \\
\hline 0.4 & 0 & 0 & 14 \\
\hline $3.5^{d}$ & 0 and $1.6^{d}$ & + & 2 \\
\hline $16^{\mathrm{e}}$ & $0^{\mathrm{e}}$ & 0 & 15 \\
\hline 21 & 3 & 0 & 17 \\
\hline 33 & 3 & $+f, g$ & 39 \\
\hline 100 & 0 & 0 & 16 \\
\hline
\end{tabular}

Estimated daily intake

(mg phenolic equivalents)

\begin{tabular}{llll} 
Olive oil & Control & Effect & Reference \\
$3.5^{\mathrm{d}}$ & 0 and $1.6^{\mathrm{d}}$ & +++ & 2 \\
\hline $7.4^{\mathrm{d}}$ & $0.7^{\mathrm{d}}$ & + & 7 \\
$8.4^{\mathrm{d}}$ & 0.1 and $3.7^{\mathrm{d}}$ & +++ & 1 \\
$11^{\mathrm{d}}$ & 0.2 and $3^{\mathrm{d}}$ & +++ & 8 \\
$13.5^{\mathrm{d}}$ & 0.1 and $6^{\mathrm{d}}$ & ++ & 13
\end{tabular}

The effect shown is based on the percentage increase in the lag time of conjugated diene formation in isolated LDL (in vitro and ex vivo studies) or the reduction in the extent of LDL oxidation measured using ELISA (in vivo studies).+ corresponds with 0-10\%, + + corresponds with $10-30 \%,+$ ++ corresponds with $30-100 \%$, and ++++ corresponds with $>100 \%$. 0 means no effect.

a. The concentration of phenolic compounds present in olive oil was expressed as $\mu \mathrm{M}$ caffeic acid equivalents; b. The protective effect was estimated from the figures presented the article; c. The protective effect of oleuropein against the formation of thiobarbituric acid-reacting substances (TBARS) and lipid hydroperoxides was presented; $d$. The daily intake was calculated using a density of olive oil of $0.92 \mathrm{~kg} / \mathrm{l}$; e. Estimated phenol intake (according to Vissers et al., ref 9); f. Susceptibility of LDL to oxidation was assessed by the rate of TBARS formation; g. Vitamin E intake was 12 vs. $8 \mathrm{mg}$ (high vs. low phenolic equivalent diet). 


\section{REFERENCES}

1. Covas MI, Nyyssonen K, Poulsen HE, et al. The effect of polyphenols in olive oil on heart disease risk factors: a randomized trial. Ann Intern Med. 2006;145(5):333-41.

2. Marrugat J, Covas MI, Fito M, et al. Effects of differing phenolic content in dietary olive oils on lipids and LDL oxidation--a randomized controlled trial. Eur J Nutr. 2004;43(3):140-7.

3. Rietjens SJ, Bast A, de Vente J, Haenen GR. The olive oil antioxidant hydroxytyrosol efficiently protects against the oxidative stress-induced impairment of the $\mathrm{NO}^{\bullet}$ response of isolated rat aorta. Am J Physiol Heart Circ Physiol. 2007;292(4):H1931-6.

4. Visioli F, Bellomo G, Galli C. Free radical-scavenging properties of olive oil polyphenols. Biochem Biophys Res Commun. 1998;247(1):60-4.

5. Visioli F, Bogani P, Grande S, Galli C. Mediterranean food and health: building human evidence. J Physiol Pharmacol. 2005;56 Suppl 1:37-49.

6. de la Torre-Carbot K, Jauregui O, Gimeno E, Castellote Al, Lamuela-Raventos RM, LopezSabater MC. Characterization and quantification of phenolic compounds in olive oils by solidphase extraction, HPLC-DAD, and HPLC-MS/MS. J Agric Food Chem. 2005;53(11):4331-40.

7. Fito $M$, Cladellas $M$, de la Torre $R$, et al. Antioxidant effect of virgin olive oil in patients with stable coronary heart disease: a randomized, crossover, controlled, clinical trial. Atherosclerosis. 2005;181(1):149-58.

8. Weinbrenner $\mathrm{T}$, Fito $\mathrm{M}$, de la Torre $\mathrm{R}$, et al. Olive oils high in phenolic compounds modulate oxidative/antioxidative status in men. J Nutr. 2004;134(9):2314-21.

9. Vissers MN, Zock PL, Katan MB. Bioavailability and antioxidant effects of olive oil phenols in humans: a review. Eur J Clin Nutr. 2004;58(6):955-65.

10. Visioli F, Bellomo G, Montedoro G, Galli C. Low density lipoprotein oxidation is inhibited in vitro by olive oil constituents. Atherosclerosis. 1995;117(1):25-32.

11. Visioli F, Galli C. Oleuropein protects low density lipoprotein from oxidation. Life Sci. 1994;55(24):1965-71.

12. Fito M, Covas MI, Lamuela Raventos RM, et al. Protective effect of olive oil and its phenolic compounds against low density lipoprotein oxidation. Lipids. 2000;35(6):633-8.

13. Covas MI, de la Torre K, Farre-Albaladejo M, et al. Postprandial LDL phenolic content and LDL oxidation are modulated by olive oil phenolic compounds in humans. Free Radic Biol Med. 2006;40(4):608-16.

14. Bonanome A, Pagnan A, Caruso D, et al. Evidence of postprandial absorption of olive oil phenols in humans. Nutr Metab Cardiovasc Dis. 2000;10(3):111-20.

15. Nicolaiew N, Lemort N, Adorni L, et al. Comparison between extra virgin olive oil and oleic acid rich sunflower oil: effects on postprandial lipemia and LDL susceptibility to oxidation. Ann Nutr Metab. 1998;42(5):251-60.

16. Vissers MN, Zock PL, Leenen R, Roodenburg AJ, van Putte KP, Katan MB. Effect of consumption of phenols from olives and extra virgin olive oil on LDL oxidizability in healthy humans. Free Radic Res. 2001;35(5):619-29.

17. Vissers MN, Zock PL, Wiseman SA, Meyboom S, Katan MB. Effect of phenol-rich extra virgin olive oil on markers of oxidation in healthy volunteers. Eur J Clin Nutr. 2001;55(5):334-41.

18. O'Dowd Y, Driss F, Dang PM, et al. Antioxidant effect of hydroxytyrosol, a polyphenol from olive oil: scavenging of hydrogen peroxide but not superoxide anion produced by human neutrophils. Biochem Pharmacol. 2004;68(10):2003-8.

19. Halliwell B, Gutteridge JM, Aruoma OI. The deoxyribose method: a simple "test-tube" assay for determination of rate constants for reactions of hydroxyl radicals. Anal Biochem. 1987;165(1):215-9.

20. den Hartog GJ, Haenen GR, Vegt E, van der Vijgh WJ, Bast A. Superoxide dismutase: the balance between prevention and induction of oxidative damage. Chem Biol Interact. 2003;145(1):33-9. 
21. Kooy NW, Royall JA, Ischiropoulos H, Beckman JS. Peroxynitrite-mediated oxidation of dihydrorhodamine 123. Free Radic Biol Med. 1994;16(2):149-56.

22. Ching TL, de Jong J, Bast A. A method for screening hypochlorous acid scavengers by inhibition of the oxidation of 5-thio-2-nitrobenzoic acid: application to anti-asthmatic drugs. Anal Biochem. 1994;218(2):377-81.

23. Haenen GR, Jansen FP, Bast A. The antioxidant properties of five O-(b-hydroxyethyl)-rutosides of the flavonoid mixture venoruton. Phlebology. 1993;suppl. 1:10-7.

24. Haenen GR, Paquay JB, Korthouwer RE, Bast A. Peroxynitrite scavenging by flavonoids. Biochem Biophys Res Commun. 1997;236(3):591-3.

25. Haenen GR, Bast A. Scavenging of hypochlorous acid by lipoic acid. Biochem Pharmacol. 1991;42(11):2244-6.

26. Paiva Martins F, Gordon MH, Gameiro P. Activity and location of olive oil phenolic antioxidants in liposomes. Chem Phys Lipids. 2003;124(1):23-36.

27. Deiana M, Aruoma OI, Bianchi ML, et al. Inhibition of peroxynitrite dependent DNA base modification and tyrosine nitration by the extra virgin olive oil-derived antioxidant hydroxytyrosol. Free Radic Biol Med. 1999;26(5-6):762-9.

28. de la Puerta R, Martinez Dominguez ME, Ruiz Gutierrez V, Flavill JA, Hoult JR. Effects of virgin olive oil phenolics on scavenging of reactive nitrogen species and upon nitrergic neurotransmission. Life Sci. 2001;69(10):1213-22.

29. Heijnen CG, Haenen GR, Vekemans JA, Bast A. Peroxynitrite scavenging of flavonoids: structure activity relationship. Environmental Toxicology and Pharmacology. 2001;10:199-206.

30. Heijnen CG, Haenen GR, Oostveen RM, Stalpers EM, Bast A. Protection of flavonoids against lipid peroxidation: the structure activity relationship revisited. Free Radic Res. 2002;36(5):57581.

31. Visioli F, Caruso D, Plasmati E, et al. Hydroxytyrosol, as a component of olive mill waste water, is dose- dependently absorbed and increases the antioxidant capacity of rat plasma. Free Radic Res. 2001;34(3):301-5.

32. Visioli F, Galli C, Bornet F, et al. Olive oil phenolics are dose-dependently absorbed in humans. FEBS Lett. 2000;468(2-3):159-60.

33. Vissers MN, Zock PL, Roodenburg AJ, Leenen R, Katan MB. Olive oil phenols are absorbed in humans. J Nutr. 2002;132(3):409-17.

34. Tuck KL, Freeman MP, Hayball PJ, Stretch GL, Stupans I. The in vivo fate of hydroxytyrosol and tyrosol, antioxidant phenolic constituents of olive oil, after intravenous and oral dosing of labeled compounds to rats. J Nutr. 2001;131(7):1993-6.

35. Miro Casas E, Covas MI, Farre M, et al. Hydroxytyrosol disposition in humans. Clin Chem. 2003;49(6 Pt 1):945-52.

36. Edgecombe SC, Stretch GL, Hayball PJ. Oleuropein, an antioxidant polyphenol from olive oil, is poorly absorbed from isolated perfused rat intestine. J Nutr. 2000;130(12):2996-3002.

37. Corona G, Tzounis X, Assunta Dessi M, et al. The fate of olive oil polyphenols in the gastrointestinal tract: implications of gastric and colonic microflora-dependent biotransformation. Free Radic Res. 2006; 40(6):647-58.

38. de la Torre-Carbot K, Chavez-Servin JL, Jauregui O, et al. Presence of virgin olive oil phenolic metabolites in human low density lipoprotein fraction: determination by high-performance liquid chromatography-electrospray ionization tandem mass spectrometry. Anal Chim Acta. 2007;583(2):402-10.

39. Ramirez Tortosa MC, Urbano G, Lopez Jurado $M$, et al. Extra-virgin olive oil increases the resistance of $L D L$ to oxidation more than refined olive oil in free-living men with peripheral vascular disease. J Nutr. 1999;129(12):2177-83. 



\section{CHAPTER 3}

The olive oil antioxidant hydroxytyrosol efficiently protects against the oxidative stress-induced impairment of the $\mathrm{NO}^{\bullet}$ response of isolated rat aorta

Saskia J. Rietjens, Aalt Bast, Jan de Vente and Guido R.M.M. Haenen

Am J Physiol Heart Circ Physiol 2007;292(4):H1931-6

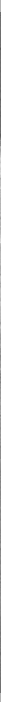




\section{ABSTRACT}

The Mediterranean diet, which is abundant in antioxidants, is associated with a relatively low incidence of coronary heart disease (CHD). Olive oil and olives, which contain the antioxidants hydroxytyrosol, oleuropein, and tyrosol, are important components of this diet. In this study, the effects of oxidative stress on the nitric oxide radical $\left(\mathrm{NO}^{*}\right)$ mediated relaxation of rat aorta and the protection by these antioxidants were determined. Cumene hydroperoxide (CHP) was used to mimic oxidative stress induced by lipid hydroperoxides, which is mediated by the formation of hydroxyl radicals $\left(\mathrm{OH}^{*}\right)$. CHP $(300 \mu \mathrm{M})$ impaired the $\mathrm{NO}^{*}$ mediated relaxation of rat aorta by the acetylcholine receptor agonist carbachol $(\mathrm{CCh})(\mathrm{P}<0.05)$. This was due to a reduction in $\mathrm{NO}^{*}$ production. A diminished $\mathrm{NO}^{\bullet}$ mediated relaxation disturbs the vascular tone and leads to a rise in blood pressure, which is a well-established risk factor for CHD. Hydroxytyrosol $(10 \mu \mathrm{M})$ efficiently protected the aorta against the CHP induced impairment of the $\mathrm{NO}^{*}$ mediated relaxation $(\mathrm{P}<0.05)$. Oleuropein, tyrosol, and homovanillic alcohol, a major metabolite of hydroxytyrosol, did not show protection. Moreover, hydroxytyrosol was found to be a potent $\mathrm{OH}^{*}$ scavenger, which can be attributed to its catechol moiety. Because of its amphiphilic characteristics (octanol-water partitioning coefficient $=1.1$ ), hydroxytyrosol will readily cross membranes and provide protection in the cytosol and membranes, including the water-lipid interface. The present study provides a molecular basis for the contribution of hydroxytyrosol to the benefits of the Mediterranean diet. 


\section{INTRODUCTION}

Epidemiological evidence has shown that the incidence of coronary heart disease (CHD) is lower in Mediterranean countries than in Western European and Northern European countries (1). In the Mediterranean area, fruits, vegetables, fish, and olive oil are important constituents of the diet and especially these constituents are thought to contribute to the protection against CHD. The relatively high antioxidant content in this traditional Mediterranean diet is seen as a key factor in the lower incidence of CHD, a disease that is related to oxidative stress. Olives and olive oil are important sources of alimentary antioxidants. Olive oil is abundant in vitamin E, flavonoids, and other phenolic antioxidants. The major phenolic compounds in olive oil are hydroxytyrosol and tyrosol. Oleuropein is present in minor quantities in olive oil and is mainly found in the olive itself (2) (Figure 1).<smiles>OCCc1ccc(O)c(O)c1</smiles>

hydroxytyrosol<smiles>OCCc1ccc(O)cc1</smiles>

tyrosol<smiles>CC=C1C(OC(C)C)OC=C(C(=O)OC)C1CC(=O)OCCc1ccc(O)c(O)c1</smiles>

oleuropein<smiles>COc1cc(CCO)ccc1O</smiles>

homovanillic alcohol

Figure 1. Molecular structure of hydroxytyrosol, oleuropein, tyrosol and homovanillic alcohol.

The aim of the present study is to investigate the effects of oxidative stress on the nitric oxide radical $\left(\mathrm{NO}^{*}\right)$ mediated vasorelaxation of isolated rat thoracic aorta. Under physiological conditions, $\mathrm{NO}^{-}$is of pivotal importance in the regulation of the vascular tone. Endothelium-derived $\mathrm{NO}^{*}$ stimulates soluble guanylyl cyclase (CG) activity in smooth muscle cells, finally leading to vasorelaxation. In the present study the lipophilic hydroperoxide cumene hydroperoxide (CHP) is used to induce oxidative stress in rat aorta. CHP is used as a model compound for lipid hydroperoxides $(\mathrm{LOOH})$, which are formed in the process of lipid peroxidation during oxidative stress. The formation of hydroxyl radicals $\left(\mathrm{OH}^{*}\right)$ by homolytic cleavage of $\mathrm{LOOH}$ induces oxidative stress. $\mathrm{OH}^{\bullet}$ is one of the most reactive chemical species known. 
The protection provided by compounds from olives and olive oil, i.e. hydroxytyrosol, oleuropein, and tyrosol, against the vascular effects induced by $\mathrm{CHP}$ is studied as well. Inasmuch as hydroxytyrosol is metabolized in vivo, also the protective effect of one of its main metabolites, homovanillic alcohol (Figure 1), is studied. To elucidate the molecular mechanism of the protection against CHP provided by the antioxidants, the potential to scavenge $\mathrm{OH}^{*}$ is determined.

\section{MATERIALS AND METHODS}

\section{Chemicals}

Hydroxytyrosol was obtained from Cayman Chemical Company, Ann Arbor, MI, USA. Oleuropein was purchased from Extrasynthese, Lyon, France. Tyrosol, homovanillic alcohol, CHP, carbachol (CCh), phenylephrine (PE), sodium nitroprusside, 2-deoxy-D-ribose, ferric chloride hexahydrate, butylated hydroxytoluene, and mannitol were obtained from Sigma-Aldrich, St Louis, USA. $\mathrm{H}_{2} \mathrm{O}_{2}$, ascorbate, 2-thiobarbituric acid, and trichloroacetic acid were purchased from Merck, Darmstadt, Germany. Anti-formaldehyde-fixed-cGMP serum was raised in sheep (3). Alexa fluor 488 donkey anti-sheep $\lg \mathrm{G}$ conjugate was obtained from Molecular Probes, Leiden, the Netherlands. All other chemicals were of analytic grade.

\section{Organ bath experiments}

The experimental protocol was approved by the Ethics Committee for Animal Experiments of the University of Maastricht. Male Lewis rats (9-11 wk old) were decapitated, the aorta was rapidly excised, and small rings ( $2 \mathrm{~mm}$ long) were mounted in thermostated organ baths $\left(37^{\circ} \mathrm{C}\right)$ containing Krebs buffer $(\mathrm{pH}$ 7.4) gassed with a mixture of $95 \% \mathrm{O}_{2}$ and $5 \% \mathrm{CO}_{2}$. Each aorta ring was connected to an isometric transducer, and the tension was adjusted to $5 \mathrm{mN}$. The composition of the Krebs buffer was (mM): $\mathrm{NaCl}$ (117.5), $\mathrm{KCl}(5.6), \mathrm{MgSO}_{4}$ (1.18), $\mathrm{CaCl}_{2}$ (2.5), $\mathrm{NaH}_{2} \mathrm{PO}_{4}$ (1.28), $\mathrm{NaHCO}_{3}$ (25), and glucose (5.5). During the experiment, the buffer was changed every $15 \mathrm{~min}$. At the beginning of the experiment, the aortic rings were washed for $60 \mathrm{~min}$.

To determine the $\mathrm{NO}^{\bullet}$ mediated relaxation, the aortic rings were first precontracted using the $\alpha_{1}$-adrenergic agonist PE. A dose-response curve of PE (from $10^{-8}$ to $3 \cdot 10^{-6} \mathrm{M}$ ) was constructed. Subsequently, the $\mathrm{NO}^{\bullet}$ mediated relaxation using the muscarinic agonist $\mathrm{CCh}$ was determined in a dose-dependent manner. The concentration CCh was increased from $10^{-8}$ to $10^{-5} \mathrm{M}$. After the construction of a reference curve of PE and $\mathrm{CCh}$ in each aorta ring, the organs 
were subjected to two 15-min washes. After they were washed, the organs were incubated for 5 min with different antioxidants (or vehicle). Hydroxytyrosol, oleuropein, and tyrosol were dissolved in ethanol, and homovanillic alcohol was dissolved in water. A relatively small volume of the compound ( $20 \mu \mathrm{l})$ was added to the organ bath $(20 \mathrm{ml})$ in the direct proximity of the aortic ring. Subsequently, the organs were incubated with CHP (or vehicle) for $30 \mathrm{~min}$. The vehicle ethanol (maximal volume of $20 \mu \mathrm{l}$ in $20 \mathrm{ml}$ of buffer) did not affect the $\mathrm{PE}$ and CCh response of the aorta.

After these incubations, the aorta was subjected to two 15-min washes, and a second dose-response curve of $\mathrm{PE}$ and $\mathrm{CCh}$ was constructed. The negative logarithm of the molar concentration that produces half-maximal effect $\left(\mathrm{pD}_{2}\right)$ and the maximal effects $\left(\mathrm{E}_{\max }\right)$ of $\mathrm{PE}$ and $\mathrm{CCh}$ were calculated. The higher the $\mathrm{pD}_{2}$, the more potent PE or CCh is. The difference in $\mathrm{E}_{\max }$ and $\mathrm{pD}_{2}$ between the first and second dose-response curve was calculated and expressed as $E_{\max }$ in percentage and $\mathrm{pD}_{2}$. The effects of $\mathrm{CHP}$ and the antioxidants on the $\mathrm{PE}$ and $\mathrm{CCh}$ response were expressed as the percentage of the control PE and CCh curves.

\section{cGMP-immunostaining of aortic tissue}

Aortic rings of 9-wk-old male Lewis rats $(n=2)$ were mounted in thermostated organ baths as described above. The organs were incubated with $100 \mu \mathrm{M}$ hydroxytyrosol (or vehicle) for $5 \mathrm{~min}$ and then with $300 \mu \mathrm{M} \mathrm{CHP}$ (or vehicle) for $30 \mathrm{~min}$. After two 15-min washes with Krebs buffer, the aortic rings were contracted by adding $3 \cdot 10^{-6} \mathrm{M}$ PE to the organ baths. Subsequently, the aortic rings were dilated with $1 \cdot 10^{-5} \mathrm{M} \mathrm{CCh}$ or $100 \mu \mathrm{M}$ sodium nitroprusside. One of the aortic rings mounted in the organ bath was left untreated during the entire experiment. This control aorta ring was not incubated with hydroxytyrosol or $\mathrm{CHP}$, nor was it contracted or dilated.

After a 6-min incubation with CCh or nitroprusside, the aortic rings were fixed for $2 \mathrm{~h}$ with ice-cold fixative solution of $4 \%$ freshly prepared depolymerised paraformaldehyde in $0.1 \mathrm{M}$ phosphate buffer $(\mathrm{pH}$ 7.4). Thereafter, the aortic rings were fixed overnight at $4^{\circ} \mathrm{C}$ in $4 \%$ paraformaldehyde containing $20 \%$ sucrose. After the overnight fixation, the aortic rings were frozen in Tissue-Tek O.C.T. compound.

A cryostat was used to cut $8-\mu \mathrm{m}$ sections, which were thawed onto chromealumn/gelatin-coated slides and then dried for $20 \mathrm{~min}$ at room temperature. After three 5-min washes with Tris-buffered saline (TBS), the sections were incubated overnight at $4^{\circ} \mathrm{C}$ with the primary antibody, i.e. sheep antiformaldehyde-fixed cGMP (1:4000) diluted in TBS containing $0.3 \%$ Triton X100 (TBS-T). The specificity of the cGMP antibody has been described elsewhere (3). 
After the incubation with primary antibody, the sections were washed, once in TBS and then twice in TBS-T, each step lasted $15 \mathrm{~min}$. For the visualization of the primary antibodies, the sections were incubated with Alexa Fluor-donkey anti-sheep $\operatorname{IgG}$ conjugate (1:100 dilution). Incubations with the secondary antibody lasted for $60 \mathrm{~min}$ in the dark at room temperature. Thereafter, sections were washed, once in TBS-T and twice in TBS, and mounted with TBS-glycerol. To check for non-specific binding of the second antibody to aortic tissue, a few slides were incubated only with the secondary antibody. These slides showed no cGMP-immunostaining.

Images were obtained at X40 magnification using a cooled CCD F-view camera on an Olympus AX70 microscope. Exposure time was held constant at $2 \mathrm{~s}$, except when sodium nitroprusside was used, where exposure time was $0.5 \mathrm{~s}$ to prevent overexposure. Two $8-\mu \mathrm{m}$ sections from each aorta ring were analysed.

\section{$\mathrm{OH}^{\bullet}$ scavenging activity}

$\mathrm{OH}^{*}$ scavenging activity was determined by the deoxyribose method according to Halliwell et al. (4). This method is based on the competition between the antioxidants and the detector molecule 2-deoxyribose for $\mathrm{OH}^{*}$ to derive the second-order rate constant for the interaction of the antioxidant with $\mathrm{OH}^{*}$. The degradation of 2-deoxyribose after reaction with $\mathrm{OH}^{\bullet}$ in a complex mixture of products results, after heating, in the formation of thiobarbituric acid-reactive material which is quantified spectrophotometrically (5). The incubation mixture contains: $\mathrm{H}_{2} \mathrm{O}_{2}(2.8 \mathrm{mM})$, 2-deoxyribose $(2.8 \mathrm{mM})$, ascorbate $(100 \mu \mathrm{M})$, and ferric chloride $(20 \mu \mathrm{M})$. In another experiment, CHP $(2.0 \mathrm{mM})$, in stead of $\mathrm{H}_{2} \mathrm{O}_{2}$, was used as a source for radicals. The protective effect of hydroxytyrosol was determined.

The second-order rate constant of the scavenger $\left(\mathrm{k}_{\mathrm{s}}\right)$ with $\mathrm{OH}^{*}$ is calculated using a rate constant of $3.1 \cdot 10^{-9} \mathrm{M}^{-1} \mathrm{~S}^{-1}$ for deoxyribose $\left(\mathrm{k}_{\mathrm{DR}}\right)(4)$. The absorbance at $532 \mathrm{~nm}$ (A) depends on the concentration of the scavenger ([S]) and the absorbance without the scavenger $\left(A_{0}\right)$. For calculation of $k_{s}$ the following formula was used:

$$
1 / A=1 / A_{0}\left(1+k_{s}[S] / k_{D R}[D R]\right)
$$

where [DR] is the concentration of 2-deoxyribose $(2.8 \mathrm{mM})$. The $\mathrm{k}_{\mathrm{s}}$ value of the scavenger is obtained from the slope of the linear plot of the reciprocal value of A vs. [S]. The $\mathrm{OH}^{\bullet}$ scavenging activity of the olive oil compounds was compared with that of the well-known $\mathrm{OH}^{*}$ scavenger mannitol. 


\section{Lipophilicity}

The lipophilicity of hydroxytyrosol was measured by determining the octanolwater partitioning coefficient $\left(\mathrm{P}_{\mathrm{o} / \mathrm{w}}\right)$. $\mathrm{P}_{\mathrm{o} / \mathrm{w}}$ of hydroxytyrosol was calculated by dividing the concentration of hydroxytyrosol in the octanol layer by that in the water layer after a $1 \mathrm{mM}$ solution of hydroxytyrosol in water that was saturated with octanol was mixed with an equal volume of octanol that was saturated with water.

\section{Statistics}

Values are means \pm SEM. Differences in the response of the aorta between the several conditions were statistically analysed performing the Student's t-test.

$\mathrm{P}<0.05$ was considered as statistically significant. Control experiments were performed on eight (no addition) and seven (CHP) rats. The experiments with hydroxytyrosol were performed on three to five rats per concentration. The experiments with the other compounds that did not show an effect were performed on two rats. For these compounds the data are presented by means \pm half range.

\section{RESULTS}

\section{Organ bath experiments}

During the incubation of the aorta with $300 \mu \mathrm{M} \mathrm{CHP}$ for 30 min, no contraction was observed. Incubation of the aorta with $300 \mu \mathrm{M}$ CHP resulted in only a small decrease in $E_{\max }$ of the $P E$ response (Figure $\left.2 A\right)(P<0.05)$. The $p D_{2}$ of the PE response was not affected after incubation with CHP (Figure 2A). A similar minor effect on the PE response was also observed after incubation with 100 $\mu \mathrm{M}$ and $1000 \mu \mathrm{M} \mathrm{CHP}$ (data not shown).

The response of the aortic rings to CCh was only slightly diminished after incubation with $100 \mu \mathrm{M} \mathrm{CHP}$, whereas it completely disappeared after incubation with $1000 \mu \mathrm{M} \mathrm{CHP}$ (data not shown). Incubation with $300 \mu \mathrm{M} \mathrm{CHP}$ significantly decreased the $\mathrm{CCh}$ response $(\mathrm{P}<0.05), \mathrm{E}_{\max }$ decreased $\sim 50 \%$ and $\mathrm{pD}_{2}$ decreased 0.71 (Figure 2B). Thus, oxidative stress, caused by incubation of the aorta with $300 \mu \mathrm{M} \mathrm{CHP}$, drastically impaired the $\mathrm{NO}^{\bullet}$ mediated relaxation by $\mathrm{CCh}$, whereas the PE response was hardly diminished. 

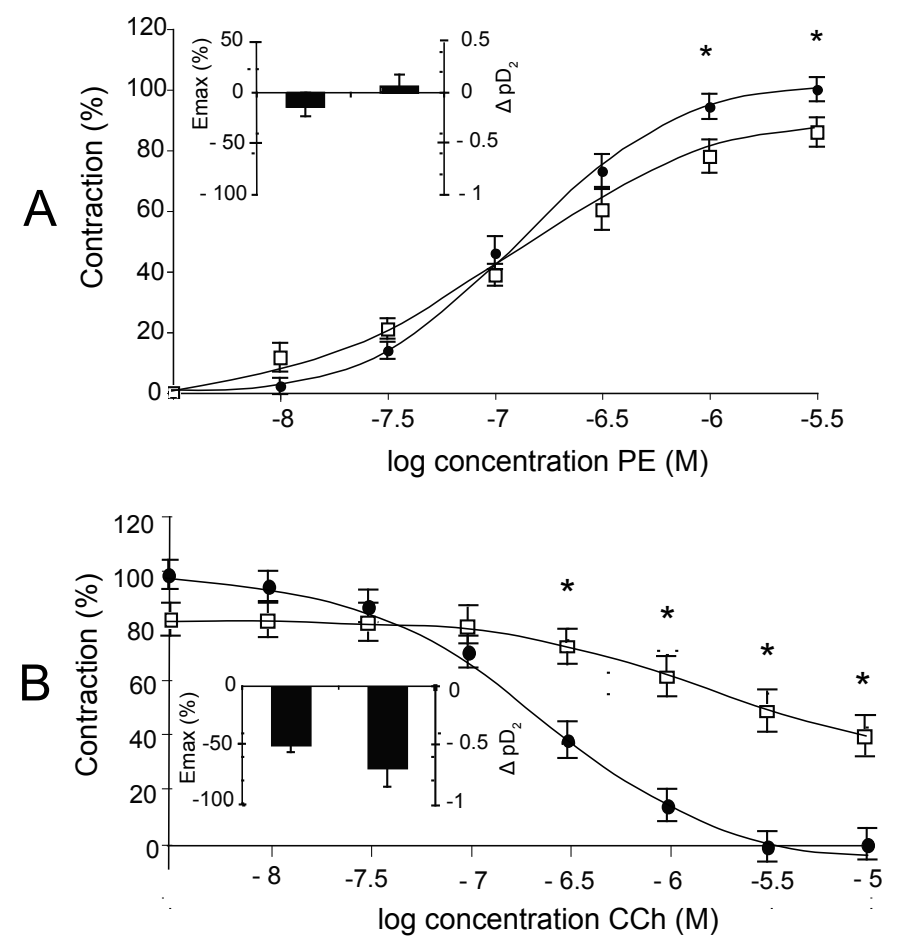

Figure 2. Effect of $300 \mu \mathrm{M} \mathrm{CHP}$ on the PE (panel A) and CCh (panel B) response. Aorta was contracted with PE (from $10^{-8}$ to $3 \cdot 10^{-6} \mathrm{M}$ ) and then dilated with CCh (from $10^{-8}$ to $10^{-5} \mathrm{M}$ ). Data points on $y$-axis show contraction of the aorta without PE (panel A) or CCh (panel B). Effects of CHP (white squares) are expressed as percentage of control (black circles) PE and CCh curves (no addition is set at $100 \%$ ). Effects of $\mathrm{CHP}$ on $\mathrm{E}_{\max }$ and $\mathrm{pD}_{2}$ of the $\mathrm{PE}$ and $\mathrm{CCh}$ response are depicted in the inserts. ${ }^{*} \mathrm{P}<0.05$

Hydroxytyrosol, oleuropein, tyrosol, and homovanillic alcohol (at $30 \mu \mathrm{M})$, had no effect on the PE and CCh response. These compounds also did not affect the minor effect of CHP on the PE response. Oleuropein, tyrosol, and homovanillic alcohol $($ at $30 \mu \mathrm{M})$ did not protect against the CHP induced reduction in the $\mathrm{NO}{ }^{\bullet}$ mediated relaxation by $\mathrm{CCh}$ (Figure 3 ). 


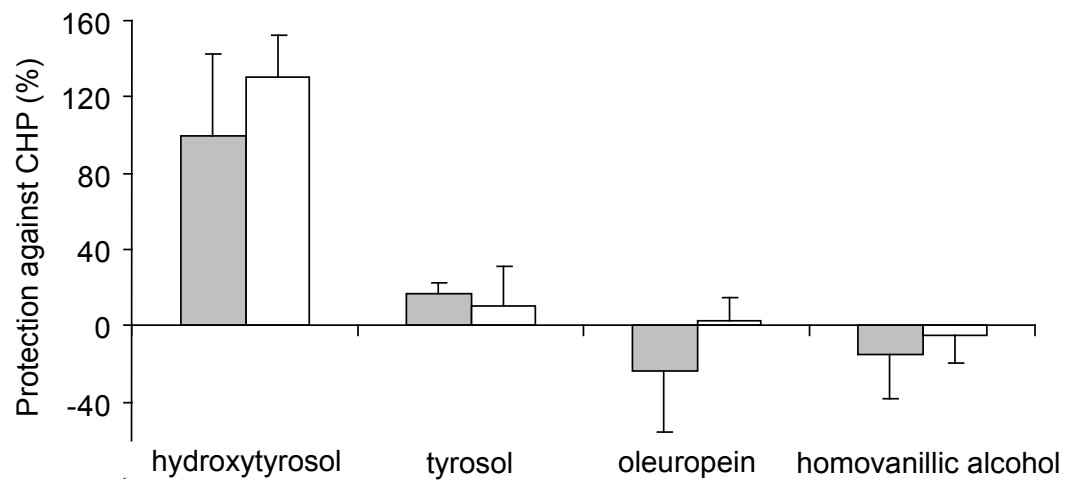

Figure 3. Protective effect of hydroxytyrosol, tyrosol, oleuropein, and homovanillic alcohol against CHP $(300 \mu \mathrm{M})$ induced impairment of the $\mathrm{NO}^{\bullet}$ mediated aortic relaxation. Values represent protection of $\mathrm{E}_{\max }$ (grey bars) and $\mathrm{pD}_{2}$ (white bars) of CCh response. Final concentration of antioxidants was $30 \mu \mathrm{M}$.

Hydroxytyrosol did protect against the effects of $300 \mu \mathrm{M}$ CHP on the $\mathrm{NO}^{*}$ mediated relaxation (Figure 3). At $30 \mu \mathrm{M}$, hydroxytyrosol completely protected against the effect of $300 \mu \mathrm{M} \mathrm{CHP}$ on $\mathrm{E}_{\max }$ of the CCh response $(\mathrm{P}<0.05)$, and $\mathrm{pD}_{2}$ of the CCh response even slightly increased $\left(\mathrm{pD}_{2}=+0.21\right)$ compared with control $(\mathrm{P}<0.05)$. The protection provided by hydroxytyrosol against the effects of $\mathrm{CHP}$ on the $\mathrm{NO}^{\bullet}$ mediated relaxation was dose dependent (Figure 4). Even relatively low concentrations of hydroxytyrosol $(10 \mu \mathrm{M}$ and $30 \mu \mathrm{M})$ protected against $300 \mu \mathrm{M} \mathrm{CHP}(\mathrm{P}<0.05)$. Hydroxytyrosol at $3 \mu \mathrm{M}$ resulted in only partial protection (Figure 4 ).

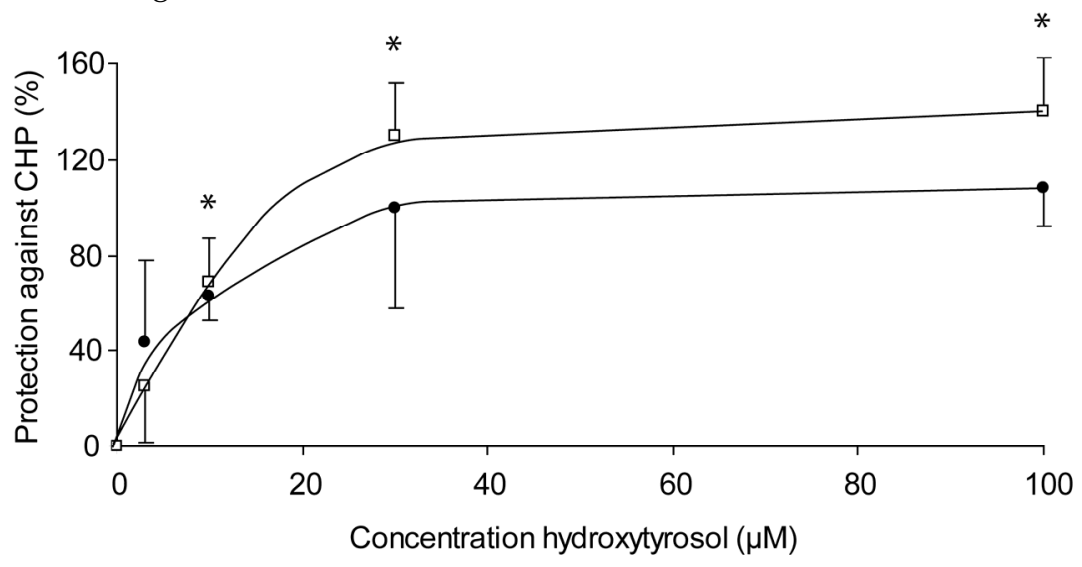

Figure 4. Dose-dependent protection of hydroxytyrosol against $\mathrm{CHP}(300 \mu \mathrm{M})$ induced impairment of $\mathrm{NO}^{*}$ mediated relaxation. Values represent protection of $\mathrm{E}_{\max }$ (black circles) and $\mathrm{pD}_{2}$ (white squares) of CCh response. Control (without CHP and hydroxytyrosol) is set at $100 \%$.

$* \mathrm{P}<0.05$ 


\section{cGMP-immunostaining of aortic tissue}

The cGMP-immunostaining of aortic tissue that was pretreated with $\mathrm{CHP}$ and subsequently contracted by PE and dilated by CCh (Figure 5B) was similar to the cGMP-immunostaining of untreated aorta (Figure 5A).

In CHP-treated aortic tissue incubated with sodium nitroprusside, cGMP accumulates in smooth muscle cells (Figure 5C). cGMP immunostaining of the aorta by $\mathrm{CCh}$ is substantially greater when the tissue is preincubated with hydroxytyrosol before the addition of CHP than when the tissue is incubated with CHP alone (Figure 5D).

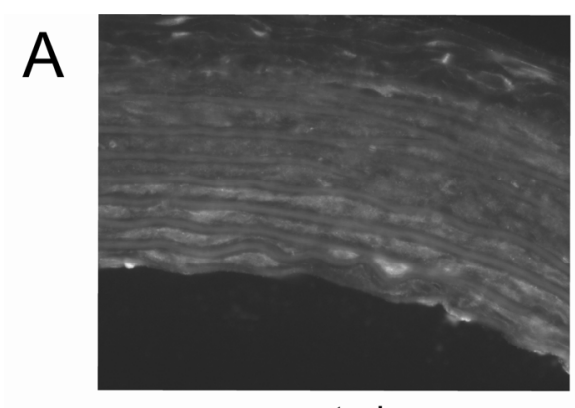

control

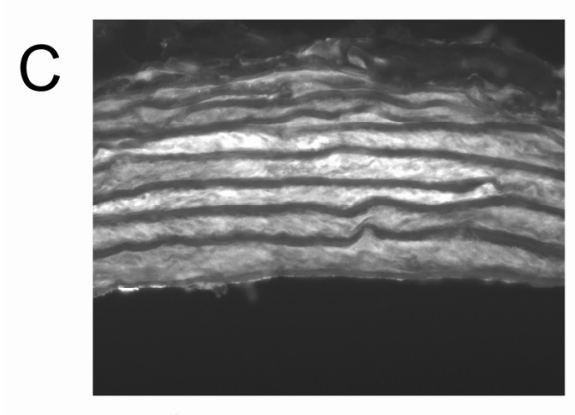

$\mathrm{CHP}+$ nitroprusside

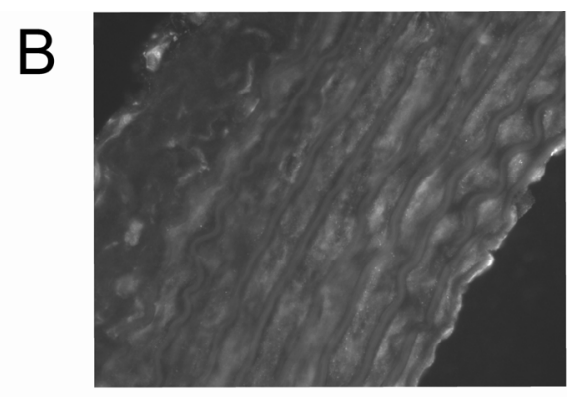

$\mathrm{CHP}+\mathrm{CCh}$

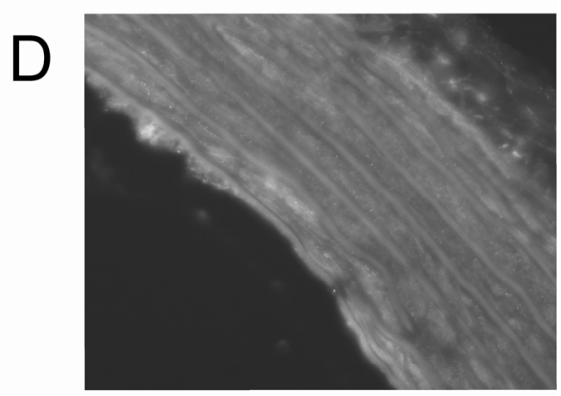

hydroxytyrosol + CHP + CCh

Figure 5. cGMP-immunostaining in rat aortic tissue. Panel A: untreated aorta (control). Aorta was incubated with $300 \mu \mathrm{M} \mathrm{CHP}$ and subsequently contracted by $\mathrm{PE}\left(3 \cdot 10^{-6} \mathrm{M}\right)$ and dilated by $\mathrm{CCh}$ $\left(10^{-5} \mathrm{M}\right)$ (panel B and D) or sodium nitroprusside $(100 \mu \mathrm{M})$ (panel C). Panel D: cGMPimmunostaining of aorta incubated with $100 \mu \mathrm{M}$ hydroxytyrosol before addition of $\mathrm{CHP}$.

\section{$\mathrm{OH}^{\bullet}$ scavenging}

To elucidate the mechanism of protection against $\mathrm{CHP}$, the ability of the compounds from olive oil to scavenge $\mathrm{OH}^{\bullet}$ was determined. Oleuropein and hydroxytyrosol were very potent $\mathrm{OH}^{\bullet}$ scavengers. The $\mathrm{OH}^{\bullet}$ scavenging activity 
was nearly seven times higher than the activity of mannitol, a well-known $\mathrm{OH}^{*}$ scavenger (Figure 6). Tyrosol and homovanillic alcohol were less effective than hydroxytyrosol in scavenging $\mathrm{OH}^{\circ}$.

When CHP was used as source for radicals in the 2-deoxyribose assay, the protective effect of hydroxytyrosol $\left(k_{s}=13.2 \cdot 10^{9} \mathrm{M}^{-1} \mathrm{~s}^{-1}\right)$ is comparable to the protection observed when $\mathrm{H}_{2} \mathrm{O}_{2}$ is used as the radical source.

To identify the part of the molecule that is responsible for the scavenging of $\mathrm{OH}^{\circ}$, the scavenging activities of the compounds were compared with the activities of catechol, 2-methoxyphenol, and phenol (Figure 6). Catechol displayed an $\mathrm{OH}^{*}$ scavenging activity comparable to that of hydroxytyrosol and oleuropein. 2-Methoxyphenol showed a somewhat higher $\mathrm{OH}^{*}$ scavenging activity than homovanillic alcohol, whereas phenol and tyrosol were equipotent.

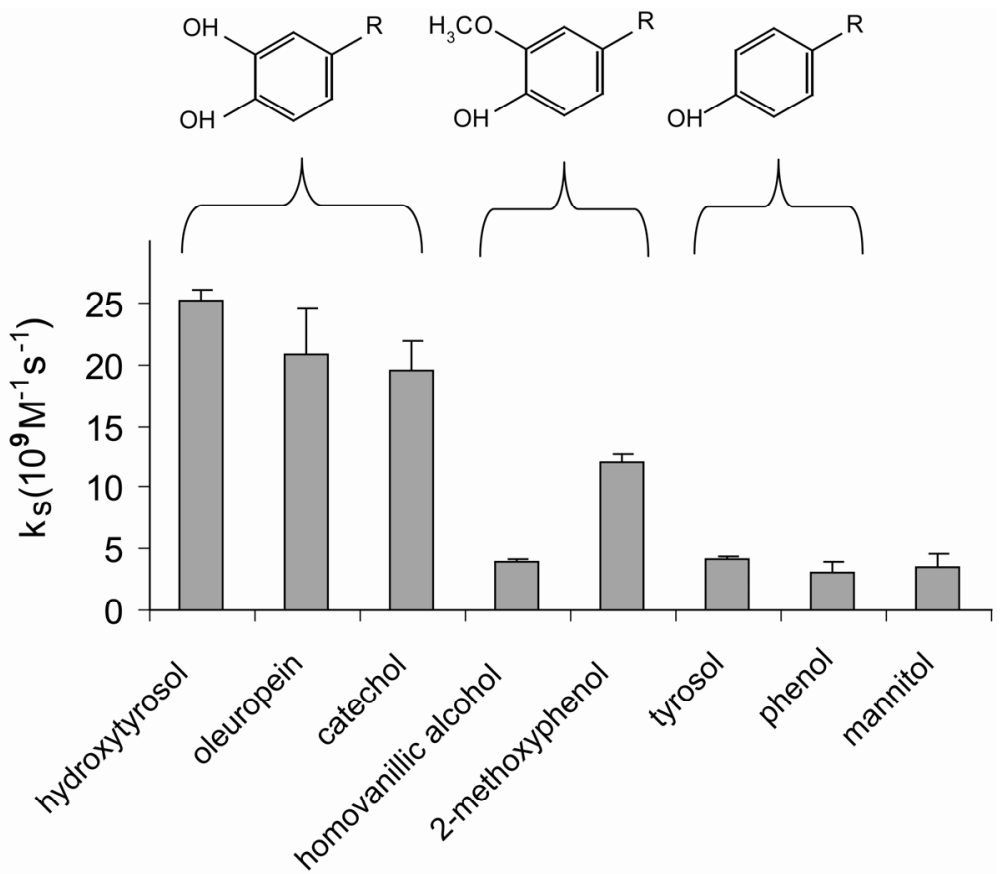

Figure 6. $\mathrm{OH}^{\bullet}$ scavenging activities of hydroxytyrosol, tyrosol, oleuropein, and homovanillic alcohol compared with mannitol and compounds sharing identical moieties. Scavenging activities are expressed as the second-order rate constant of the scavenger $\left(\mathrm{k}_{\mathrm{s}}\right)$ with $\mathrm{OH}^{*}$.

\section{Lipophilicity}

Lipophilicity is a key factor in many biological effects of compounds. The lipophilicity of hydroxytyrosol was measured by determination of $\mathrm{P}_{\mathrm{o} / \mathrm{w}}$ which 
was found to be 1.1. This means that the concentration of hydroxytyrosol is expected to be very similar in the water and the lipid phase.

\section{DISCUSSION}

In the present study the effect of oxidative stress on the $\mathrm{NO}^{\bullet}$ mediated relaxation of rat aorta was determined. During oxidative stress, polyunsaturated fatty acids (PUFAs), present in, e.g., membranes of endothelial and smooth muscle cells in blood vessels, are oxidatively damaged. This process is called lipid peroxidation. During this process, $\mathrm{LOOH}$ are formed. These $\mathrm{LOOH}$ amplify the oxidative stress, because they readily form radicals, e.g., the extremely reactive $\mathrm{OH}^{\bullet}$ by homolytic cleavage of the $\mathrm{O}-\mathrm{O}$ bond.

In the present study, it was shown that CHP, a model compound for LOOH, drastically impaired the $\mathrm{NO}^{\bullet}$ mediated relaxation by $\mathrm{CCh} . \mathrm{pD}_{2}$ decreased 0.7 after incubation with $\mathrm{CHP}$, which means that the acetylcholine receptormediated response decreased $80 \%$. The $\mathrm{E}_{\max }$ decreased $\sim 50 \%$ after incubation with $\mathrm{CHP}$, which implies that the smooth muscle itself is also affected. Endothelium-derived $\mathrm{NO}^{\circ}$ is an important regulator of vascular tone. Damage to the $\mathrm{NO}^{\bullet}$ regulating mechanism is involved in hypertension and the development or progression of atherosclerosis $(6,7)$. CHP hardly decreased the PE response, which means that the $\alpha_{1}$ adrenoceptor mediated contraction is not affected.

Hydroxytyrosol very efficiently protected the aorta of the rat against the CHP induced impairment of the $\mathrm{NO}^{\bullet}$ mediated relaxation: 1 molecule of hydroxytyrosol is able to provide protection against 30 molecules of CHP. Therefore, hydroxytyrosol has to be considered as an excellent antioxidant.

To further study the inhibitory effect of $\mathrm{CHP}$ on the CCh induced relaxation, we investigated the effect of CHP on cGMP levels in aortic tissue. The addition of sodium nitroprusside to CHP-treated aortic tissue significantly increased cGMP levels in smooth muscle cells. Therefore, the inhibitory effect of CHP on the CCh induced relaxation of the aorta cannot be explained by inactivation of GC activity in smooth muscle cells. This indicates that CHP most likely impairs the release of $\mathrm{NO}^{\bullet}$ from the endothelium.

We demonstrated that preincubation with hydroxytyrosol before the addition of $\mathrm{CHP}$ results in substantially more cGMP-immunostaining in aortic tissue by $\mathrm{CCh}$ than does incubation with $\mathrm{CHP}$ alone. These results support the hypothesis that hydroxytyrosol protects against the oxidative stress induced impairment of the $\mathrm{NO}^{\bullet}$ mediated vasorelaxation.

Hydroxytyrosol is metabolized in the body by the action of catechol-Omethyltransferase, an enzyme also involved in catecholamine catabolism (8). One of its major metabolites, homovanillic alcohol, provided no protection 
against the $\mathrm{CHP}$ induced effects on the $\mathrm{NO}^{\bullet}$ mediated relaxation. Also, oleuropein and tyrosol were not able to protect the aorta against oxidative stress.

$\mathrm{CHP}$ induces oxidative damage by the generation of $\mathrm{OH}^{\circ}$. To explain the difference in protection against oxidative stress between hydroxytyrosol, oleuropein, homovanillic alcohol, and tyrosol, the ability of the compounds to scavenge $\mathrm{OH}^{*}$ was determined. Hydroxytyrosol and oleuropein were potent scavengers of $\mathrm{OH}^{*}$ compared with homovanillic alcohol and tyrosol. The high $\mathrm{OH}^{*}$ scavenging activity of hydroxytyrosol and oleuropein can be attributed to the presence of a catechol moiety in their molecular structure.

In catechol, each ortho $\mathrm{OH}$ group has a high electron-donating effect on the other $\mathrm{OH}$ group. Weakening of the $\mathrm{OH}$ bond by this electron donation facilitates transfer a hydrogen atom to $\mathrm{OH}^{*}$, converting the $\mathrm{OH}^{*}$ into water $(9,10)$. The electron-donating effect of an ortho-methoxy group is also relatively high but significantly lower than that of an ortho-hydroxy group (9). Subsequently, methylation of an $\mathrm{OH}$ group in a catechol moiety will reduce the electrondonating effect. This explains the lower $\mathrm{OH}^{*}$ scavenging activity of homovanillic alcohol. Tyrosol lacks a potent electron-donating substituent, explaining its poor $\mathrm{OH}^{\bullet}$ scavenging activity.

The finding that hydroxytyrosol very efficiently protects the aorta against $\mathrm{CHP}$ is consistent with its high $\mathrm{OH}^{*}$ scavenging activity. The observed second- order rate constant of hydroxytyrosol $\left(k_{s}\right)$ with $\mathrm{OH}^{*}$ in our assay is even higher than the diffusion rate constant. A rate constant higher than the diffusion rate constant can be explained by iron chelation. By chelating iron, hydroxytyrosol is present exactly at the site of $\mathrm{OH}^{*}$ generation. This phenomenon has been called site-specific scavenging (5). Iron is also involved in the generation of $\mathrm{OH}^{\bullet}$ from CHP in the organ bath model. Apparently, in these experiments, hydroxytyrosol is also present at exactly the right place, i.e. at exactly the site where the radical is formed.

Hydroxytyrosol has an amphiphilic structure $\left(\mathrm{P}_{\mathrm{o} / \mathrm{w}} \sim 1\right)$, which means that its concentration in cytosol and membranes is practically the same. $P_{o / w}$ of 1.1 indicates that hydroxytyrosol will readily cross membranes. The amphiphilic nature also indicates that hydroxytyrosol will provide protection in the cytosol and membranes, including the water-lipid interface. From the difference in protection of the aorta against CHP between hydroxytyrosol and oleuropein, it appears that the presence of a catechol moiety does not guarantee an efficient antioxidant activity. The hydrophilic sugar moiety in oleuropein probably prevents oleuropein from crossing membranes. Therefore, it cannot protect against CHP within smooth muscle or endothelial cells. The sugar moiety also explains its poor bioavailability. The relative amount of orally administered oleuropein that reaches the systemic circulation unchanged is small. In vivo, oleuropein can be metabolized into the aglycon hydroxytyrosol in the intestine or the liver (11). 
Amphiphilic compounds, such as hydroxytyrosol, are generally well absorbed. Several studies indeed report that the uptake of hydroxytyrosol is good (12-14). The consumption of olive oil in the Mediterranean area is high compared with Western European and Northern European countries. For instance, in Greece the mean yearly intake is $\sim 15 \mathrm{~kg}$ per person. Consumption of a real-life dose of $25 \mathrm{ml}$ of olive oil per day (containing $\sim 1 \mathrm{mg}$ of hydroxytyrosol) leads to a plasma concentration of $50 \mathrm{nM}$ (15) to $160 \mathrm{nM}$ (16).

The hydroxytyrosol concentrations tested in our study are higher than those obtained with consumption of olive oil. A relatively high concentration of CHP $(300 \mu \mathrm{M})$ was required to induce oxidative damage in a relatively short time span (17). This acute in vitro model is used to mimic long-lasting oxidative stress in vivo, sometimes lasting even more than a decade. A relatively low concentration of hydroxytyrosol $(10 \mu \mathrm{M})$ protected well against the excess of $\mathrm{CHP}(300 \mu \mathrm{M})$.

The level of oxidative stress in the body is far lower than that generated in the organ baths. This indicates that less hydroxytyrosol than that used in our in vitro study will be needed in vivo to protect against oxidative stress. Thus hydroxytyrosol levels reached by the Mediterranean diet are expected to protect against oxidative stress in vivo. Moreover, it is quite possible that, in vivo, hydroxytyrosol has additive or synergistic effects with endogenous antioxidants or other antioxidants that are abundantly present in the Mediterranean diet.

Various reports on beneficial effects of hydroxytyrosol have been published $(18,19)$. For example, it has been shown that hydroxytyrosol is able to protect against the oxidation of low-density lipoprotein (LDL) (20), one of the key steps in the initiation of atherosclerosis. In a recent nutritional trial in humans, it was shown that partial substitution of carbohydrate with olive oil products lowered blood pressure, improved lipid levels, and reduced the estimated cardiovascular risk (21). These effects were attributed to the high content of monounsaturated fatty acids in the diet. The results of the present study indicate that phenolic compounds present in olive oil might also contribute to the blood pressure-lowering effect. The beneficial effect of phenolic compounds on endothelial function is supported by a recent study of Ruano et al., in which they showed that the intake of high-phenolic olive oil, when compared to lowphenolic olive oil, improved endothelial function in patients with hypercholesterolemia (22).

In conclusion, hydroxytyrosol is very efficient in protecting the aorta against the oxidative stress induced impairment of the $\mathrm{NO}^{*}$ mediated relaxation. $\mathrm{NO}^{*}$ is of pivotal importance in the regulation of the vascular tone. The high potency of hydroxytyrosol can be explained by 1 ) its amphiphilic nature, which results in a good absorption and the presence of hydroxytyrosol in membranes and cytosol, and 2) its efficient radical-scavenging activity, which involves site specific 
scavenging. The present study provides a molecular basis for the contribution of hydroxytyrosol to the benefits of the Mediterranean diet.

Acknowledgements

We thank Marie-José Drittij and Gregorio Fazzi for their practical assistance. 


\section{REFERENCES}

1. Keys A, Menotti A, Karvonen MJ, et al. The diet and 15-year death rate in the seven countries study. Am J Epidemiol. 1986;124(6):903-15.

2. Amiot M, Fleuriet A. Importance and evolution of phenolic compounds in olive during growth and maturation. J. Agric. Food. Chem. 1986;34:823-826.

3. Tanaka J, Markerink-van Ittersum M, Steinbusch HW, De Vente J. Nitric oxide-mediated cGMP synthesis in oligodendrocytes in the developing rat brain. Glia. 1997;19(4):286-97.

4. Halliwell B, Gutteridge JM, Aruoma OI. The deoxyribose method: a simple "test-tube" assay for determination of rate constants for reactions of hydroxyl radicals. Anal Biochem. 1987;165(1):215-9.

5. Haenen GR, Jansen FP, Bast A. The antioxidant properties of five O-(b-hydroxyethyl)-rutosides of the flavonoid mixture venoruton. Phlebology. 1993;suppl. 1:10-7.

6. Ross R. Atherosclerosis--an inflammatory disease. N Engl J Med. 1999;340(2):115-26.

7. Kinlay S, Ganz P. Relation between endothelial dysfunction and the acute coronary syndrome: implications for therapy. Am J Cardiol. 2000;86(8B):10J-13J; discussion 13J-14J.

8. Caruso D, Visioli F, Patelli R, Galli C, Galli G. Urinary excretion of olive oil phenols and their metabolites in humans. Metabolism. 2001;50(12):1426-8.

9. Heijnen CG, Haenen GR, Vekemans JA, Bast A. Peroxynitrite scavenging of flavonoids: structure activity relationship. Environmental Toxicology and Pharmacology. 2001;10:199-206.

10. Heijnen CG, Haenen GR, Oostveen RM, Stalpers EM, Bast A. Protection of flavonoids against lipid peroxidation: the structure activity relationship revisited. Free Radic Res. 2002;36(5):57581.

11. Edgecombe SC, Stretch GL, Hayball PJ. Oleuropein, an antioxidant polyphenol from olive oil, is poorly absorbed from isolated perfused rat intestine. J Nutr. 2000;130(12):2996-3002.

12. Visioli F, Caruso D, Plasmati E, et al. Hydroxytyrosol, as a component of olive mill waste water, is dose- dependently absorbed and increases the antioxidant capacity of rat plasma. Free Radic Res. 2001;34(3):301-5.

13. Visioli F, Galli C, Bornet F, et al. Olive oil phenolics are dose-dependently absorbed in humans. FEBS Lett. 2000;468(2-3):159-60.

14. Vissers MN, Zock PL, Roodenburg AJ, Leenen R, Katan MB. Olive oil phenols are absorbed in humans. J Nutr. 2002;132(3):409-17.

15. Weinbrenner $T$, Fito $M$, de la Torre $R$, et al. Olive oils high in phenolic compounds modulate oxidative/antioxidative status in men. J Nutr. 2004;134(9):2314-21.

16. Miro Casas E, Covas MI, Farre M, et al. Hydroxytyrosol disposition in humans. Clin Chem. 2003;49(6 Pt 1):945-52.

17. Haenen GR, Veerman M, Bast A. Reduction of beta-adrenoceptor function by oxidative stress in the heart. Free Radic Biol Med. 1990;9(4):279-88.

18. Vissers MN, Zock PL, Katan MB. Bioavailability and antioxidant effects of olive oil phenols in humans: a review. Eur J Clin Nutr. 2004;58(6):955-65.

19. Tuck KL, Hayball PJ. Major phenolic compounds in olive oil: metabolism and health effects. J. Nutr. Biochem. 2002;13:636-644.

20. Visioli F, Bellomo G, Montedoro G, Galli C. Low density lipoprotein oxidation is inhibited in vitro by olive oil constituents. Atherosclerosis. 1995;117(1):25-32.

21. Appel LJ, Sacks FM, Carey VJ, et al. Effects of protein, monounsaturated fat, and carbohydrate intake on blood pressure and serum lipids: results of the OmniHeart randomized trial. Jama. 2005;294(19):2455-64.

22. Ruano J, Lopez-Miranda J, Fuentes F, et al. Phenolic content of virgin olive oil improves ischemic reactive hyperemia in hypercholesterolemic patients. J Am Coll Cardiol. 2005;46(10):1864-8. 


\section{ABSTRACT}

During exercise, the production of reactive oxygen and nitrogen species significantly increases. The aim of the present study was to investigate the effects of a single session of resistance exercise on antioxidant capacity, oxidative damage, and inflammation. Muscle biopsies, urine, and blood samples were collected from seven healthy men before and after a single bout of resistance exercise. A single session of resistance exercise was found to induce oxidative damage, as shown by a $40 \%$ increase in the concentration of urinary $\mathrm{F}_{2 \alpha}$-isoprostanes $(\mathrm{P}<0.05)$. Total antioxidant capacity (TEAC) of plasma increased $16 \%(\mathrm{P}<0.05)$. This increase seemed to be predominantly attributable to an increase in plasma uric acid concentrations of $53 \%(\mathrm{P}<0.05)$. Similar to uric acid, but to a relatively much smaller extent, vitamin $\mathrm{C}$ and vitamin $\mathrm{E}$ levels in plasma were also elevated $(\mathrm{P}<0.05)$. Moreover, the erythrocyte glutathione $(\mathrm{GSH})$ concentration increased $47 \%$ during exercise $(\mathrm{P}<0.05)$. Also in skeletal muscle, uric acid levels were found to increase after exercise $(\mathrm{P}<0.05)$. Moreover, thirty minutes after exercise, skeletal muscle glutathione $\mathrm{S}$ transferase (GST) and glutathione reductase activity increased $28 \%$ and $42 \%$, respectively $(\mathrm{P}<0.05)$. Skeletal muscle reduced glutathione $(\mathrm{GSH})$ and glutathione disulphide (GSSG) concentrations were not affected by exercise. The Nuclear Factor kappa B (NF-kB) activity in peripheral blood mononuclear cells (PBMCs) was not increased by exercise, indicating that a NF-KB mediated inflammatory response does not occur. We conclude that a single session of resistance exercise induces oxidative damage, despite an adaptive increase in antioxidant capacity of blood and skeletal muscle. 


\section{INTRODUCTION}

The production of reactive oxygen species (ROS) and reactive nitrogen species is significantly enhanced during exercise. For example, superoxide radical $\left(\mathrm{O}_{2}{ }^{-}\right)$production by mitochondria is increased (1). Other potential sources for ROS generation during exercise are phospholipase $A_{2}$ dependent processes, plasma membrane nicotinamide adenine dinucleotide phosphate (NADPH) oxidases, and ischemia reperfusion (2-4). During ischemia, xanthine dehydrogenase is converted into xanthine oxidase, an enzyme producing $\mathrm{O}_{2}{ }^{-}$especially during reperfusion. Injury to skeletal muscle tissue, such as that induced by exercise, is accompanied by invasion of the injured tissue with phagocytic cells. This further promotes the production of ROS and activates proinflammatory pathways such as NF- $\mathrm{kB}$.

Fortunately, the body has an elaborate antioxidant defence system that provides protection against these reactive species. The antioxidant defence system can be subdivided into enzymatic antioxidants, such as glutathione reductase and glutathione S-transferase (GST), and non-enzymatic antioxidants, such as uric acid, glutathione (GSH), vitamin $\mathrm{C}$ and vitamin $\mathrm{E}$.

Nonetheless, when the production of reactive species exceeds the protection provided by antioxidants, oxidative stress occurs. This can result in oxidative damage to biomolecules such as proteins and lipids. For example, several studies have shown that exercise induces lipid peroxidation, as demonstrated by an increase in thiobarbituric acid reactive substances (TBARS), isoprostanes and exhaled pentane (5). In nearly all of these studies, endurance exercise was applied (5). Far less information is available on resistance exercise-induced oxidative stress (6-13).

The aim of the present study is to investigate whether the antioxidant defence system is able to cope with the increased radical flux induced by resistance exercise. More specifically, we investigated in untrained healthy males the diverse effects of a single session of resistance exercise on antioxidant activity, oxidative damage, and inflammation.

\section{MATERIALS AND METHODS}

\section{Subjects}

Eight healthy male volunteers with no history of participating in any regular exercise program were recruited for the present study. One of the subjects was excluded from the statistical analysis because he could not comply with the exercise protocol and had to stop before all the repetitions were performed. 
Subjects' characteristics are shown in Table 1. All subjects were informed on the nature and possible risks of the experimental procedures before their written informed consent was obtained. This study was approved by the Medical Ethical Review Board of the Academic Hospital, Maastricht, the Netherlands.

Table 1. Subjects' characteristics

\begin{tabular}{lc}
\hline & Mean \pm SEM \\
\hline Age $(\mathrm{yrs})$ & $22.4 \pm 2.0$ \\
Body mass $(\mathrm{kg})$ & $74.4 \pm 3.3$ \\
Height $(\mathrm{m})$ & $1.82 \pm 0.03$ \\
BMl $\left(\mathrm{kg} \cdot \mathrm{m}^{-2}\right)$ & $22.3 \pm 0.8$ \\
\hline
\end{tabular}

Values are expressed as mean \pm SEM.

\section{Pretesting}

Two weeks before the experimental trial, the subjects performed a short exercise session to become familiarized with the exercise protocol and the equipment. Proper weightlifting technique was demonstrated and practiced for each of the two lower-limb exercises (leg press and leg extension). Subsequently, maximum strength was estimated using a standard multiple repetitions testing procedure (14). After warming up, the load was gradually increased, and the successful lifts until failure were determined. A five-minute resting period between subsequent attempts was allowed.

In another session, at least one week after the first session and at least one week before the experimental trial, subjects' one repetition maximum (1RM) for each of the two lower-limb exercises was determined (15). The 1RM of the leg extension was determined initially, followed by that of the leg press.

After warming up, the load was set at $90 \%$ of the estimated $1 \mathrm{RM}$, and was increased after each successful lift until failure. Thereafter, the load was increased by $2.5-5 \%$ and the successful lifts until failure were determined. A 5min resting period between subsequent attempts was allowed. A repetition was valid if the subject was able to complete the entire lift in a controlled manner without assistance. 1RM was typically reached within three attempts.

Approximately 16 and 8 repetitions were performed during the first and second exercise sessions, respectively. The two sessions were kept as short as possible to prevent training effects.

\section{Standardization of diet and activity prior to testing}

Subjects were instructed not to consume any products rich in antioxidants, such as fruit juices, chocolate, olive oil, wine, or antioxidant supplements during three days before testing, on the test day itself, and on the day after the test day until $24 \mathrm{~h}$ after exercise. The low-antioxidant diet was chosen firstly to 
minimize a potential protective effect of high antioxidant intake and, secondly, to reduce variation in the baseline level of antioxidants, such as vitamin $\mathrm{E}$ and vitamin C. The volunteers consumed a carbohydrate-rich noodle dish, containing approximately $10 \mathrm{~g}$ of the vegetable leek, at $8.00 \mathrm{pm}$ in the evening before the test day. Subjects were asked to record their food intake for three days before testing, on the test day itself, and on the day after the test day until $24 \mathrm{~h}$ after exercise. Food choices and quantities were recorded. By reviewing the recorded food intake, it was verified that the subjects' diet complied with the instructions. The volunteers were instructed to refrain from any sort of heavy physical exercise during the entire period, except for the resistance exercise session.

\section{Experimental trial}

The subjects arrived at the laboratory at $8.00 \mathrm{am}$, in an overnight fasted state. Then, they performed a 5-min low-intensity warm up using a Stairmaster (Jimsa Benelux BV, Rotterdam, the Netherlands). Thereafter, the resistance exercise session targeted the legs, with eight sets of ten repetitions on the horizontal leg press machine (Technogym BV, Rotterdam, the Netherlands) and eight sets of ten repetitions on the leg extension machine (Technogym). The leg press and leg extension were performed in random order so that two subjects could be tested simultaneously. The starting workload applied during resistance exercise session was $75 \%$ of the individual $1 \mathrm{RM}$ for both the leg press and leg extension with 2-min rest intervals between sets. In total, the exercise regime required 41 min to complete. This resistance exercise protocol has been published previously and is routinely used as a resistance type of exercise in our institute (14, 15).

If subjects could not finish all ten repetitions at full weight, this was reduced to 65 or $55 \%$ of the individual $1 \mathrm{RM}$. Only one of the evaluated volunteers was able to finish the entire protocol at $75 \%$. The average intensity level of the exercise regime was $70 \%$. All subjects were verbally encouraged during the test to complete the entire protocol.

\section{Blood sampling}

A Teflon catheter was inserted in an antecubital vein for venous blood sampling. Blood was collected before the start of the exercise $(t=0)$, during exercise $(\mathrm{t}=20 \mathrm{~min}), 2 \mathrm{~min}$ after cessation of exercise $(\mathrm{t}=43 \mathrm{~min}$ ) and during subsequent recovery ( $\mathrm{t}=60 \mathrm{~min}, 90 \mathrm{~min}, 120 \mathrm{~min}, 150 \mathrm{~min}, 180 \mathrm{~min}$, and $24 \mathrm{~h}$ after exercise). Blood samples were collected in tubes containing heparin and placed on ice. Blood was aliquoted for the vitamin $\mathrm{C}$ and glutathione and glutathione disulphide (GSH/GSSG) analysis. To preserve the samples, 5\% 
trichloroacetic acid (TCA) was added to the former, and $1.3 \%$ sulphosalicylic acid (SSA) was added to the latter. After centrifugation at $1000 \cdot \mathrm{g}$ at $4{ }^{\circ} \mathrm{C}$ for 5 min, aliquots of plasma were stored at $-80^{\circ} \mathrm{C}$ until analysis.

\section{Blood analyses}

Plasma lactate concentrations were analysed spectrophotometrically (340 nm), measuring $\mathrm{NADH}$ formation using lactate dehydrogenase (LDH) (16).

The total antioxidant capacity (TEAC) was determined in plasma that was deproteinized with a final concentration of 5\% TCA (17). The samples were incubated with the ABTS radical solution for $5 \mathrm{~min}$ and subsequently the absorbance at $734 \mathrm{~nm}$ was measured. The TEAC is expressed as $\mu \mathrm{M}$ trolox equivalents.

Uric acid was determined in plasma that was deproteinized with a final concentration of 5\% TCA, using HPLC. A Hypersil BDS C-18 end-capped column, $125 \times 4 \mathrm{~mm}$, particle size $5 \mu \mathrm{m}$ (Agilent, Palo Alto, CA, USA), was used, with a mobile phase of $0.1 \%$ trifluoroacetic acid $(\mathrm{v} / \mathrm{v})$ in water. UV detection was performed at $292 \mathrm{~nm}$. The relative contribution of uric acid to the TEAC is calculated using the TEAC value of 1 for uric acid (18).

Vitamin $\mathrm{C}$ was determined in plasma by HPLC using the following method. Vitamin $\mathrm{C}$ was oxidized by ascorbate oxidase to dehydroascorbate. The latter was condensed with o-phenylene diamine to its quinoxaline derivative. This derivative was quantified on the same system used for uric acid with a mobile phase of $0.08 \mathrm{M}$ phosphate buffer $(\mathrm{pH} 7.8)$ and methanol, with fluorimetric detection (355-425 nm).

Vitamin E was extracted using hexane after adding vitamin E nicotinate as internal standard. HPLC analysis was performed on the same system used for uric acid with a mobile phase of methanol. UV detection was used (295 nm).

The erythrocyte non-protein thiol concentration, representing mainly GSH, was measured using 5,5'-dithiobis-2-nitrobenzoic acid (DTNB). The erythrocyte GSSG concentration was measured using 2-vinylpyridine pre-treatment.

\section{NF-кB activity in peripheral blood mononuclear cells (PBMCs)}

PBMCs were isolated from the blood samples (collected into leucosep tubes at $\mathrm{t}=0$, $43 \mathrm{~min}, 90 \mathrm{~min}$, and $24 \mathrm{~h}$ after exercise) using Lymphoprep (Bio-Connect, Huissen, the Netherlands). Nuclear extracts were immediately prepared and stored at $-80^{\circ} \mathrm{C}$ until analysis. The protein concentration was determined according to the method of Bradford (BioRad, Veenendaal, the Netherlands).

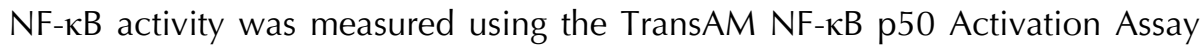
(Active Motif, Rixensart, Belgium) and expressed as $\mu$ g Jurkat NF-kB equivalents per $\mu$ g nuclear protein. 


\section{Urine collection}

Morning urine was collected on the test day and on the morning of the day after the test day. Aliquots of urine were stored at $-80^{\circ} \mathrm{C}$ until analysis of $\mathrm{F}_{2 \alpha^{-}}$ isoprostanes and creatinine.

\section{Urine analysis}

$\mathrm{F}_{2 \alpha}$-isoprostanes (8-iso $\mathrm{PGF}_{2 \alpha}$ ) in urine were analysed by an enzyme immunoassay (Cayman Chemical, Ann Arbor, MI, USA) and were related to creatinine values.

\section{Muscle biopsies}

Muscle biopsy samples were taken 30 min before the start of the exercise and 30 min after exercise, to determine the acute effects of exercise. Muscle biopsies were obtained from the middle region of the $\mathrm{m}$. vastus lateralis $(15 \mathrm{~cm}$ above the patella) and approximately $3 \mathrm{~cm}$ below entry through the fascia using the percutaneous needle biopsy technique (19). The second muscle biopsy was taken from the contra lateral leg. Muscle samples were freed from any visible nonmuscle material and were rapidly frozen in liquid nitrogen. Muscle biopsies were stored at $-80^{\circ} \mathrm{C}$ until analysis.

\section{Preparation of muscle homogenates}

Frozen muscle biopsies were weighed and subsequently ground with a liquid nitrogen cooled mortar. The powder was resuspended in demineralised water to a final concentration of $250 \mathrm{mg}$ tissue/ml. SSA (final concentration of 1.3\%) was added to a small part of the homogenates, for the analysis of GSH and GSSG. The homogenates were directly frozen in liquid nitrogen and were subsequently stored at $-80^{\circ} \mathrm{C}$ until analysis. The homogenates were centrifuged at $14,000 \cdot \mathrm{g}$ at $4^{\circ} \mathrm{C}$ for $3 \mathrm{~min}$. The muscle biopsy supernatant was used for analysis.

\section{Muscle biopsy analyses}

GST activity, glutathione reductase activity, uric acid and protein levels in muscle biopsies were determined according to the methods of Julicher et al. (20). GST activity was determined by the enzymatic reaction of chlorodinitrobenzene with GSH. The increase in the formation of the coloured product of the reaction was measured at $340 \mathrm{~nm}$ for $2 \mathrm{~min}$. Glutathione reductase activity was measured by determining the consumption of NADPH in the enzymatic reduction of GSSG to GSH. The decrease in absorption at $340 \mathrm{~nm}$ was meas- 
ured for 2 min. Skeletal muscle GSH and GSSG concentrations were measured according to the method for the determination of erythrocyte GSH and GSSG concentrations, as described above.

Uric acid concentrations were determined in muscle biopsies of six subjects. In general, all analyses were performed within a month after sample collection.

\section{Statistics}

All data are expressed as means \pm SEM. The non-parametric, repeatedmeasures ANOVA of Friedman was applied to determine differences over time in plasma antioxidant concentrations, lactate levels, and NF-kB activity. In cases of significant differences over time, a post hoc test, described by Siegel and Castellan (21), was used to locate the differences in time (vs $t=0$ ). The Wilcoxon signed-rank test was used to compare pre- $(t=0)$ versus post-exercise values (muscle biopsies, urine and $24 \mathrm{~h}$ plasma). Statistical significance was set at $\mathrm{P}<0.05$.

\section{RESULTS}

\section{Resistance exercise}

Mean 1 RM measured during pretesting was $195 \pm 11 \mathrm{~kg}$ on the horizontal leg press and $117 \pm 6 \mathrm{~kg}$ on the leg extension. The starting workload applied during resistance exercise averaged $146 \pm 8 \mathrm{~kg}$ and $88 \pm 5 \mathrm{~kg}(75 \%$ of $1 \mathrm{RM})$ for the leg press and leg extension, respectively. For most of the participants, this workload had to be reduced, as specified in the material and method section. The average intensity level of the exercise regime was $70 \%$.

\section{Lactate}

Plasma lactate levels markedly increased during exercise $(\mathrm{P}<0.05)$ and subsequently decreased during recovery (Figure 1).

Plasma lactate concentrations during exercise and subsequent recovery were identical to those reported by Koopman et al. (15), who applied the same exercise protocol. Twenty-four hours after exercise, lactate levels were slightly elevated compared with baseline levels $(1.39 \pm 0.21 \mathrm{mM}$ at $24 \mathrm{~h}$ vs $0.86 \pm$ $0.08 \mathrm{mM}$ at $\mathrm{t}=0 ; \mathrm{P}<0.05)$. 


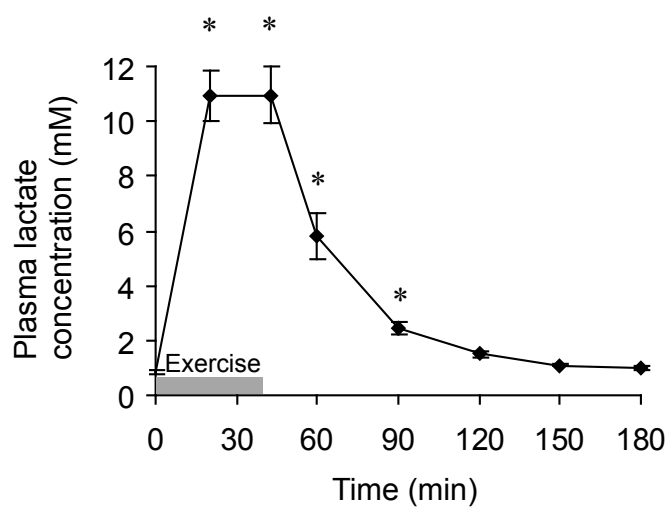

Figure 1. Plasma lactate levels at baseline, during resistance exercise (duration is indicated by the grey bar) and subsequent recovery. Data provided are means \pm SEM.

* Significantly different from pre-exercise values at baseline $(\mathrm{P}<0.05)$.

\section{Plasma antioxidant capacity}

Plasma TEAC increased during exercise and reached maximal values $50 \mathrm{~min}$ after exercise (maximal increment of $16 \pm 4 \%$; Figure 2A). Twenty-four hours after exercise, TEAC values remained elevated compared with baseline values $(784 \pm 19 \mu \mathrm{M}$ at $24 \mathrm{~h}$ vs. $743 \pm 19 \mu \mathrm{M}$ trolox equivalents at $\mathrm{t}=0$; $\mathrm{P}<0.05)$. Plasma uric acid concentrations gradually increased during exercise. After exercise, the uric acid concentration increased up to $153 \pm 13 \%$ ( $t=90 \mathrm{~min}$; $\mathrm{P}<0.05)$, after which it slightly decreased (Figure $2 \mathrm{~B}$ ). The uric acid concentration remained elevated even up to $24 \mathrm{~h}$ after exercise $(366 \pm 32 \mu \mathrm{M}$ at $24 \mathrm{~h}$ vs. $295 \pm 21 \mu \mathrm{M}$ at $\mathrm{t}=0 ; \mathrm{P}<0.05)$. The antioxidant uric acid significantly contributes to the TEAC. Subtracting the contribution of uric acid from the TEAC gives the 'uric acid-subtracted TEAC' value. The 'uric acid-subtracted TEAC' markedly decreased during exercise with a maximum decline of $14 \pm 2 \%$ after exercise (Figure 2C). Twenty-four hours after exercise, the 'uric acid-subtracted TEAC' remained below baseline $(419 \pm 19 \mu \mathrm{M}$ at $24 \mathrm{~h}$ vs. $449 \pm 7 \mu \mathrm{M}$ trolox equivalents at $\mathrm{t}=0 ; \mathrm{P}<0.05)$.

Plasma vitamin $\mathrm{C}$ concentrations elevated from $15.3 \pm 0.6 \mu \mathrm{M}$ at baseline to $16.4 \pm 0.7 \mu \mathrm{M}$ after exercise $(\mathrm{t}=43 \mathrm{~min} ; \mathrm{P}<0.05)$. Also at $\mathrm{t}=150$ and $180 \mathrm{~min}$ vitamin $\mathrm{C}$ concentrations were elevated compared to baseline $(\mathrm{P}<0.05)$ (Figure $3 \mathrm{~A})$. Twenty-four hours after exercise, plasma vitamin $\mathrm{C}$ concentrations were equal to those at baseline $(15.3 \pm 0.5 \mu \mathrm{M}$ at $24 \mathrm{~h}$ vs. $15.3 \pm 0.6 \mu \mathrm{M}$ at $\mathrm{t}=0)$. Plasma vitamin E concentrations increased from $10.0 \pm 0.4 \mu \mathrm{g} / \mathrm{ml}$ at baseline to $10.9 \pm 0.5 \mu \mathrm{g} / \mathrm{ml}$ during exercise $(\mathrm{t}=20 \mathrm{~min})(\mathrm{P}<0.05)$ and returned to baseline levels during recovery (Figure $3 \mathrm{~B}$ ). Plasma vitamin $\mathrm{E}$ concentrations were slightly elevated $24 \mathrm{~h}$ after exercise $(10.5 \pm 0.5 \mu \mathrm{g} / \mathrm{ml}$ at $24 \mathrm{~h}$ vs. $10.0 \pm 0.4$ $\mu \mathrm{g} / \mathrm{ml}$ at $\mathrm{t}=0 ; \mathrm{P}<0.05)$. 

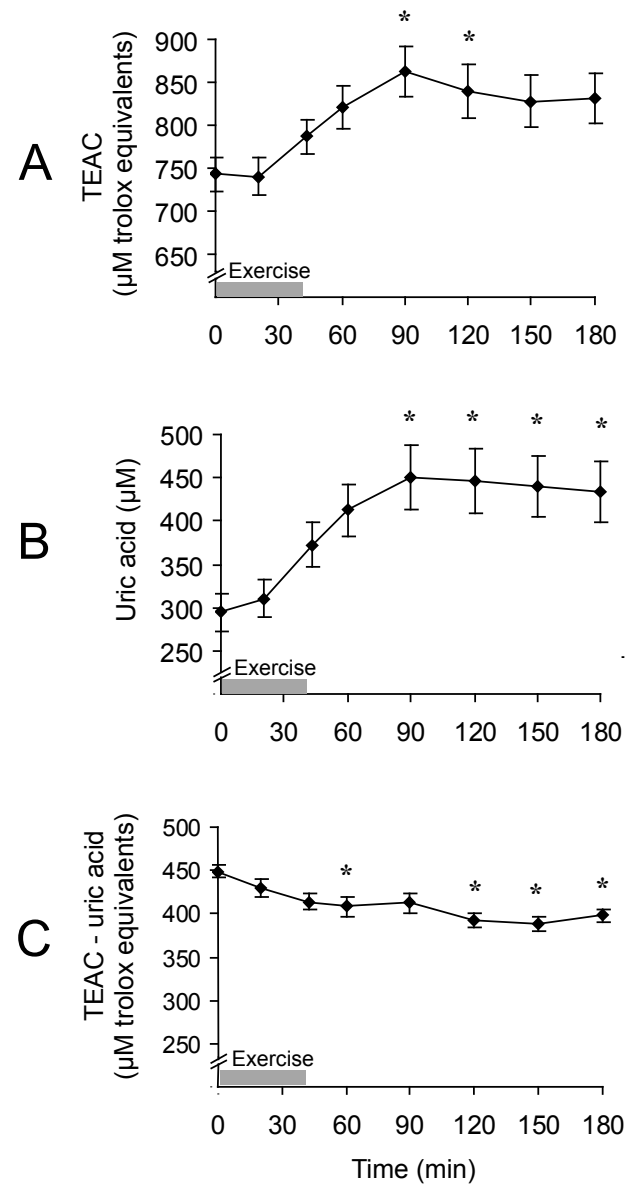

Figure 2. Plasma TEAC values (panel A), uric acid concentrations (panel B) and TEAC values, corrected for uric acid (panel C), at baseline, during resistance exercise (duration is indicated by the grey bar) and subsequent recovery. Data provided are means \pm SEM.

* Significantly different from pre-exercise values at baseline $(\mathrm{P}<0.05)$.

\section{NF-кB activity}

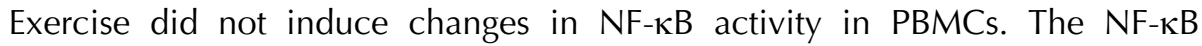
activity at $\mathrm{t}=43 \mathrm{~min}, \mathrm{t}=90 \mathrm{~min}$, and $24 \mathrm{~h}$ (respectively $0.49 \pm 0.01,0.52 \pm 0.02$

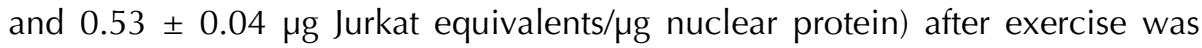
similar to the NF- $\kappa \mathrm{B}$ activity at baseline $(0.53 \pm 0.04 \mu \mathrm{g}$ Jurkat equivalents $/ \mu \mathrm{g}$ nuclear protein). 

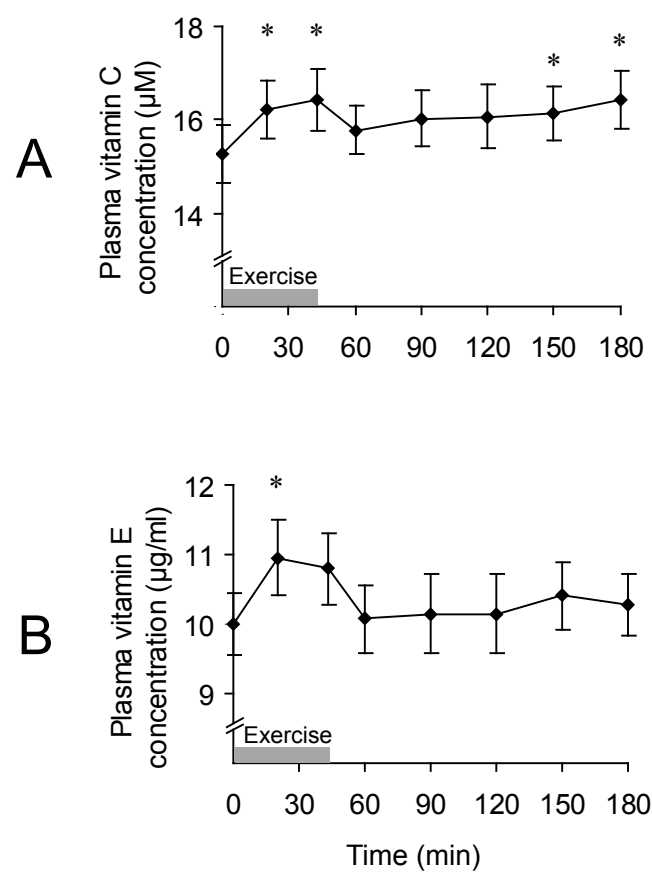

Figure 3. Plasma vitamin C (panel A) and vitamin E (panel B) concentrations during resistance exercise (duration is indicated by the grey bar) and subsequent recovery. Data provided are means \pm SEM. * Significantly different from pre-exercise values at baseline $(\mathrm{P}<0.05)$.

\section{8-iso PGF $_{2 a}$ levels in urine}

The concentration 8-iso $\mathrm{PGF}_{2 \alpha}$ in urine increased from $0.117 \pm 0.021$ $\mathrm{nmol} / \mathrm{mmol}$ creatinine pre-exercise to $0.164 \pm 0.030 \mathrm{nmol} / \mathrm{mmol}$ creatinine post-exercise $(\mathrm{P}<0.05)$. $\quad \mathrm{F}_{2 \alpha}$-isoprostanes are prostaglandin-like biomarkers specific for ROS-mediated peroxidation of arachidonic acid, a poly unsaturated fatty acid (PUFA) present, for example, in membranes (22).

\section{Antioxidant activity in skeletal muscle}

Uric acid concentrations in skeletal muscle increased from $82 \pm 6 \mu \mathrm{mol} / \mathrm{kg}$ muscle pre-exercise to $106 \pm 9 \mu \mathrm{mol} / \mathrm{kg}$ muscle $30 \mathrm{~min}$ after exercise $(\mathrm{P}<0.05)$. GST activity in skeletal muscle increased from $0.224 \pm 0.032 \mathrm{U} / \mathrm{mg}$ protein at rest to $0.286 \pm 0.014 \mathrm{U} / \mathrm{mg}$ protein $30 \mathrm{~min}$ after exercise $(\mathrm{P}<0.05$; Figure 4). Glutathione reductase activity in skeletal muscle increased from $10.4 \pm 1.8$ $\mathrm{mU} / \mathrm{mg}$ protein at rest to $14.8 \pm 2.4 \mathrm{mU} / \mathrm{mg}$ protein $30 \mathrm{~min}$ after exercise $(\mathrm{P}<0.05$; Figure 4). 


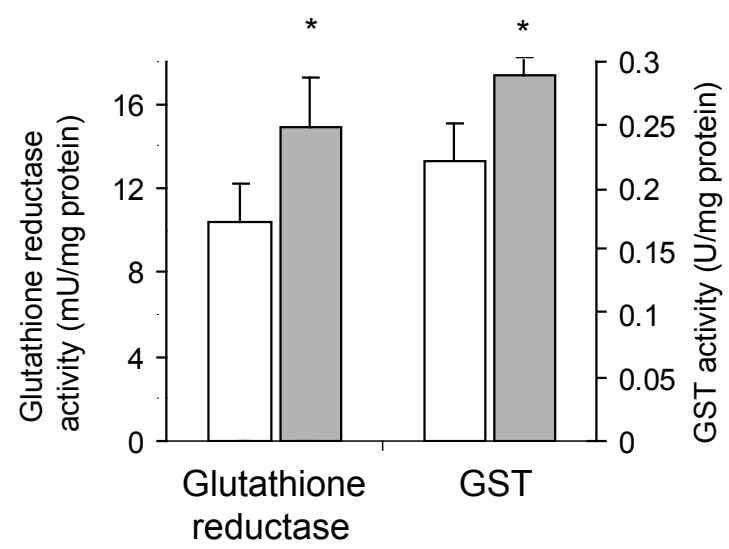

Figure 4. Glutathione reductase and GST activities in skeletal muscle at baseline (white bars) and 30 min after exercise (grey bars). Data provided are means \pm SEM.

* Significantly different from pre-exercise values at baseline $(\mathrm{P}<0.05)$.

GSH and GSSG concentrations in skeletal muscle were not affected by exercise. In contrast, erythrocyte GSH concentrations increased $47 \%$ during exercise, from $10.9 \pm 2.4 \mathrm{nmol} / \mathrm{mg} \mathrm{Hb}$ at baseline to $16.0 \pm 1.9 \mathrm{nmol} / \mathrm{mg} \mathrm{Hb}$ during exercise ( $\mathrm{t}=20 \mathrm{~min} ; \mathrm{P}<0.05)$, and returned to baseline values during recovery (Figure 5). The erythrocyte GSSG concentration remained at baseline value.

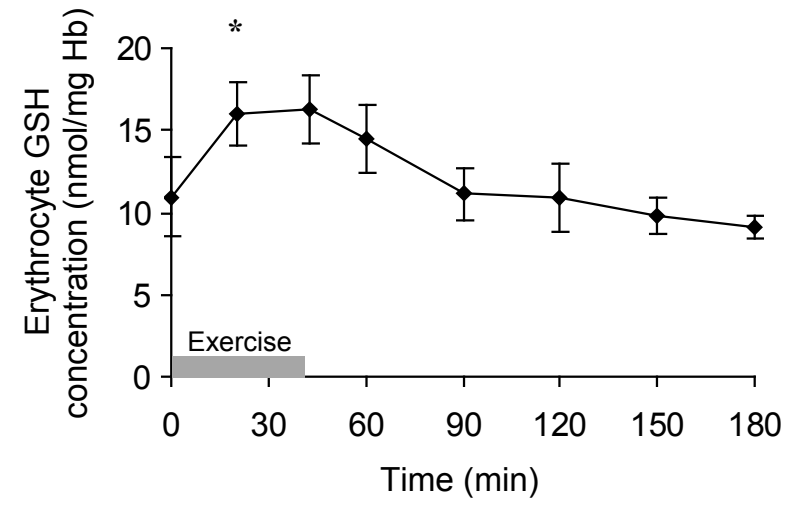

Figure 5. Erythrocyte GSH concentrations at baseline, during exercise (duration is indicated by the grey bar) and subsequent recovery. Data provided are means \pm SEM.

* Significantly different from pre-exercise values at baseline $(\mathrm{P}<0.05)$. 


\section{DISCUSSION}

Exercise enhances the production of ROS. The aim of the present study was to investigate whether the body's antioxidant defence system is able to cope with the increased radical flux induced by a single session of resistance exercise.

Antioxidants protect against radicals by converting them to chemically less reactive species. In this scavenging reaction, the antioxidant becomes oxidized or chemically modified in another way. Therefore, it was expected that antioxidant levels would be reduced by increased radical production during exercise. However, in the present study, a single session of resistance exercise was found to increase the concentration of several antioxidants.

The increase in the total antioxidant capacity (TEAC) of plasma appeared to be predominantly due to elevated uric acid concentrations. A similar increase in plasma uric acid by exercise has been reported previously (23). Also in skeletal muscle, uric acid levels were found to increase after exercise.

The increase in uric acid might be caused by the exercise-induced breakdown of ATP, as well as the activation of xanthine oxidase that forms, besides $\mathrm{O}_{2}{ }^{-}$, also uric acid. The inhibition of renal clearance of uric acid by lactate accumulation may also be responsible for the rise in uric acid level (24). In the present study it was shown that lactate levels rose significantly during resistance exercise. The elevated uric acid levels persisted a long time after exercise. This can be explained by the relatively long half-life of uric acid of approximately 20 hours (25). The rise in uric acid levels might be a physiological mechanism to counteract an increased radical production by exercise.

Similar to uric acid, vitamin $C$ and vitamin $E$ levels in plasma were also elevated during exercise. In the case of vitamin $\mathrm{E}$, this has previously been reported (8). This relatively small increase in the concentrations of vitamin $\mathrm{C}$ and vitamin $E$ has a negligible effect on the plasma TEAC value.

In contrast to vitamin $C$ and vitamin $E$, uric acid substantially contributes to the TEAC. Subtracting the contribution of uric acid from the TEAC gives the "uric acid-subtracted TEAC' value. Resistance exercise induces a decrease in this 'uric acid-subtracted TEAC', which indicates that other antioxidants responsible for the TEAC are consumed during exercise. This consumption of antioxidants confirms that radicals have been produced by the applied resistance exercise regime.

Exercise induced a large increase in the concentration of GSH in erythrocytes. Similar to the rise in uric acid, this might be an adaptive response to increased radical production. The origin of the increase in GSH is unclear. In skeletal muscle, no increase in GSH was found.

The activity of the antioxidant enzymes GST and glutathione reductase in skeletal muscle was shown to significantly increase by a single session of resistance exercise. In rodents, antioxidant enzymes in skeletal muscle have 
also been reported to increase, especially after repetitive prolonged bouts of exercise (26). The enhancement in skeletal muscle antioxidant enzyme activity probably involves the activation of the antioxidant responsive element (ARE) pathway by exercise-induced radical formation (27). Similar to the rise in uric acid and erythrocyte GSH levels, this can also be regarded as an adaptive response to increased radical production.

The rise in urinary $\mathrm{F}_{2 \alpha}$-isoprostanes indicates that resistance exercise induces oxidative damage. This indicates that the adaptive responses in blood and skeletal muscle do not provide full protection against the increased radical flux induced by the applied resistance exercise.

Injury to skeletal muscle tissue is accompanied by invasion of the area with phagocytic cells, which further promotes the production of ROS and activates pro-inflammatory pathways such as NF-kB. The results of the present study indicate that the oxidative damage induced by a single bout of resistance exercise does not induce a NF-kB mediated inflammatory response in untrained healthy volunteers.

No consensus exists on the effect of resistance exercise on markers of oxidative stress (6). This topic has been reviewed in detail by Bloomer and Goldfarb (6). Some studies show that resistance exercise induces oxidative damage $(6,8,10$ 12 ), by demonstrating increased levels of malondialdehyde (MDA) or protein carbonyls, while other studies show no increase in oxidative damage $(6,7,9)$. This discrepancy might be explained by a number of factors, such as differences in biomarkers studied, the type and duration of resistance exercise or whether or not subjects were trained. For example, chronic anaerobic training might enhance the endogenous antioxidant defence (6), which might attenuate exercise-induced oxidative damage. Nevertheless, in trained individuals, resistance exercise has also been reported to induce oxidative damage to proteins or lipids $(10,11,28)$.

A negative health effect of a high radical flux induced by exercise is expected to be more pronounced in individuals with a compromised antioxidant defence system $(29,30)$, such as in COPD or type 2 diabetes. Besides an impaired antioxidant defence, these patients have a relatively high production of radicals even under basal conditions $(31,32)$. Interestingly, exercise is recommended in the treatment of these patients. Antioxidant supplementation during exercise might especially be relevant for these patients, as it strengthens the antioxidant defence against ROS generated during exercise. Antioxidant supplementation should not affect the positive health effects of exercise. Although free radicals are typically considered as destructive species, current evidence indicates that in specific situations some reactive species might have a beneficial health effect (2).

Despite the exercise-induced adaptive increase in antioxidant capacity of blood and skeletal muscle, a single session of resistance exercise induces oxidative 
damage in healthy men. The challenge is 1) to unravel the mechanisms that underlie both the positive and negative health effects of ROS generated during exercise and 2) to selectively block those pathways that have negative health effects without affecting the beneficial processes. Undoubtedly, antioxidants will come into play.

Acknowledgements

We would like to thank Mike Coleman, Nico Crombach, Marie-José Drittij, Marc Fischer, Ralph Manders, and Bart Pennings for their valuable assistance. 


\section{REFERENCES}

1. Sjodin B, Hellsten Westing Y, Apple FS. Biochemical mechanisms for oxygen free radical formation during exercise. Sports Med. 1990;10(4):236-54.

2. Reid MB. Invited Review: redox modulation of skeletal muscle contraction: what we know and what we don't. J Appl Physiol. 2001;90(2):724-31.

3. Finaud J, Lac G, Filaire E. Oxidative stress : relationship with exercise and training. Sports Med. 2006;36(4):327-58.

4. Jackson MJ, Pye D, Palomero J. The production of reactive oxygen and nitrogen species by skeletal muscle. J Appl Physiol. 2007;102(4):1664-1670.

5. Vollaard NB, Shearman JP, Cooper CE. Exercise-induced oxidative stress:myths, realities and physiological relevance. Sports Med. 2005;35(12):1045-62.

6. Bloomer RJ, Goldfarb AH. Anaerobic exercise and oxidative stress: a review. Can J Appl Physiol. 2004;29(3):245-63.

7. Akova B, Surmen-Gur E, Gur H, Dirican M, Sarandol E, Kucukoglu S. Exercise-induced oxidative stress and muscle performance in healthy women: role of vitamin $E$ supplementation and endogenous oestradiol. Eur J Appl Physiol. 2001;84(1-2):141-7.

8. Ramel A, Wagner KH, Elmadfa I. Plasma antioxidants and lipid oxidation after submaximal resistance exercise in men. Eur J Nutr. 2004;43(1):2-6.

9. Bloomer RJ, Falvo MJ, Fry AC, Schilling BK, Smith WA, Moore CA. Oxidative stress response in trained men following repeated squats or sprints. Med Sci Sports Exerc. 2006;38(8):1436-42.

10. Bloomer RJ, Fry AC, Falvo MJ, Moore CA. Protein carbonyls are acutely elevated following single set anaerobic exercise in resistance trained men. J Sci Med Sport. 2007;10(6):411-7.

11. Bloomer RJ, Goldfarb AH, Wideman L, McKenzie MJ, Consitt LA. Effects of acute aerobic and anaerobic exercise on blood markers of oxidative stress. J Strength Cond Res. 2005;19(2):27685.

12. Goldfarb AH, Bloomer RJ, McKenzie MJ. Combined antioxidant treatment effects on blood oxidative stress after eccentric exercise. Med Sci Sports Exerc. 2005;37(2):234-9.

13. Bryer SC, Goldfarb AH. Effect of high dose vitamin C supplementation on muscle soreness, damage, function, and oxidative stress to eccentric exercise. Int J Sport Nutr Exerc Metab. 2006;16(3):270-80.

14. Koopman R, Manders RJ, Zorenc AH, et al. A single session of resistance exercise enhances insulin sensitivity for at least $24 \mathrm{~h}$ in healthy men. Eur J Appl Physiol. 2005;94(1-2):180-7.

15. Koopman R, Manders RJ, Jonkers RA, Hul GB, Kuipers H, van Loon LJ. Intramyocellular lipid and glycogen content are reduced following resistance exercise in untrained healthy males. Eur J Appl Physiol. 2006;96(5):525-34.

16. Gutmann I, Wahlefeld A. L-(+)-Lactate, determination with lactate dehydrogenase and NAD. In: Bergmeyer H, ed. Methods in enzymatic analysis. 2 ed. New York: Academic; 1974:14641468.

17. Fischer MA, Gransier TJ, Beckers LM, Bekers O, Bast A, Haenen GR. Determination of the antioxidant capacity in blood. Clin Chem Lab Med. 2005;43(7):735-40.

18. Arts MJ, Dallinga JS, Voss HP, Haenen GR, Bast A. A new approach to assess the total antioxidant capacity using the TEAC assay. Food Chem. 2004;88:567-570.

19. Bergstrom J. Percutaneous needle biopsy of skeletal muscle in physiological and clinical research. Scand J Clin Lab Invest. 1975;35(7):609-16.

20. Julicher RH, Sterrenberg L, Haenen GR, Bast A, Noordhoek J. Sex differences in the cellular defence system against free radicals from oxygen or drug metabolites in rat. Arch Toxicol. 1984;56(2):83-6.

21. Siegel S, Castellan N. Nonparametric statistics for the behavioral sciences. 2 ed New York: McGraw-Hill; 1988.

22. Griffiths HR, Moller L, Bartosz G, et al. Biomarkers. Mol Aspects Med. 2002;23(1-3):101-208. 
23. Green HJ, Fraser IG. Differential effects of exercise intensity on serum uric acid concentration. Med Sci Sports Exerc. 1988;20(1):55-9.

24. Ames BN, Cathcart R, Schwiers E, Hochstein P. Uric acid provides an antioxidant defense in humans against oxidant- and radical-caused aging and cancer: a hypothesis. Proc Natl Acad Sci U S A. 1981;78(11):6858-62.

25. Geren W, Bendich A, Bodansky O, Brown GB. The fate of uric acid in man. J Biol Chem. 1950;183(1):21-31.

26. Powers SK, Ji LL, Leeuwenburgh C. Exercise training-induced alterations in skeletal muscle antioxidant capacity: a brief review. Med Sci Sports Exerc. 1999;31(7):987-97.

27. Nguyen T, Sherratt PJ, Pickett CB. Regulatory mechanisms controlling gene expression mediated by the antioxidant response element. Annu Rev Pharmacol Toxicol. 2003;43:233-60.

28. McBride JM, Kraemer WJ, Triplett-McBride T, Sebastianelli W. Effect of resistance exercise on free radical production. Med Sci Sports Exerc. 1998;30(1):67-72.

29. Leaf DA, Kleinman MT, Hamilton M, Deitrick RW. The exercise-induced oxidative stress paradox: the effects of physical exercise training. Am J Med Sci. 1999;317(5):295-300.

30. Villa-Caballero L, Nava-Ocampo AA, Frati-Munari A, Ponce-Monter H. Oxidative stress, acute and regular exercise: are they really harmful in the diabetic patient? Med Hypotheses. 2000;55(1):43-6.

31. Maritim AC, Sanders RA, Watkins JB, 3rd. Diabetes, oxidative stress, and antioxidants: a review. J Biochem Mol Toxicol. 2003;17(1):24-38.

32. Boots AW, Haenen GR, Bast A. Oxidant metabolism in chronic obstructive pulmonary disease. Eur Respir J Suppl. 2003;46:14s-27s. 



\section{CHAPTER 5}

The olive oil antioxidant hydroxytyrosol protects against
oxidative stress-induced reduction in
skeletal muscle force production

Saskia J. Rietjens, Aalt Bast and Guido R.M.M. Haenen

Submitted

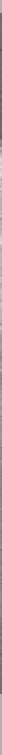




\section{ABSTRACT}

The aim of the present study was to investigate the protective effect of the olive oil antioxidant hydroxytyrosol against oxidative stress-induced impairment of skeletal muscle contractile function. The isolated rat diaphragm was used to study skeletal muscle function in vitro. Hydroxytyrosol concentrationdependently protected the muscle strips against the $\mathrm{H}_{2} \mathrm{O}_{2}$-induced impairment in force production during short-term stimulation at a wide range of stimulation frequencies $(10-100 \mathrm{~Hz})$. A relatively low concentration of hydroxytyrosol (25

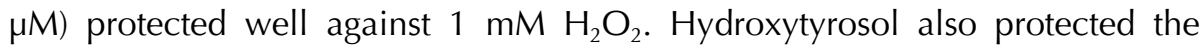
muscle strips against the $\mathrm{H}_{2} \mathrm{O}_{2}$-induced impairment of force production during especially the initial stage of a long-term fatigue protocol. The protection provided by hydroxytyrosol was not based on a direct $\mathrm{H}_{2} \mathrm{O}_{2}$ scavenging effect. $\mathrm{H}_{2} \mathrm{O}_{2}$ can be converted to other, much more reactive species, such as the highly reactive hydroxyl radical $\left(\mathrm{OH}^{*}\right)$. The excellent antioxidant efficacy of hydroxytyrosol is illustrated by its potent $\mathrm{OH}^{*}$ scavenging activity. The in vivo impact of the high antioxidant potency of hydroxytyrosol might not only lie in the field of muscle protection during strenuous exercise, but also in conditions such as ventilatory insufficiency or chronic fatigue syndrome. 


\section{INTRODUCTION}

Skeletal muscle fibres continuously generate reactive oxygen species (ROS) at a slow rate (1). This rate increases during muscle contraction. Over the past decades, progress has been made in the understanding of both the beneficial and detrimental roles that ROS play in skeletal muscle. Low levels of ROS have been implicated in optimal muscle contractile function (1). However, a high and unbalanced production of ROS will lead to oxidative damage (2-4). ROS act indiscriminately. Some ROS are able to damage virtually any cellular component, including proteins, lipids and DNA.

ROS can affect skeletal muscle force production by damaging proteins that are important in regulating cytosolic free $\mathrm{Ca}^{2+}$, such as the sarcoplasmic reticulum ryanodine receptor (RYR) and $\mathrm{Ca}^{2+}$ ATPases (5). Moreover, ROS are also able to modify an array of other regulatory proteins in skeletal muscle including myofibrillar proteins $(6,7)$, mitochondrial proteins (8) and proteins that regulate glucose metabolism (9). The detrimental effects of ROS provide the rationale for antioxidant supplementation to reduce oxidative muscle damage and the fatigability of muscle cells.

Hydroxytyrosol (Figure 1), a phenolic compound present in virgin olive oil, has been shown to be a very effective antioxidant (10). For example, it has been reported that hydroxytyrosol efficiently protects vascular tissue against oxidative stress (11). Moreover, hydroxytyrosol is able to reduce oxidative damage in intestinal epithelial cells (12), hepatocytes (13), and erythrocytes (14).

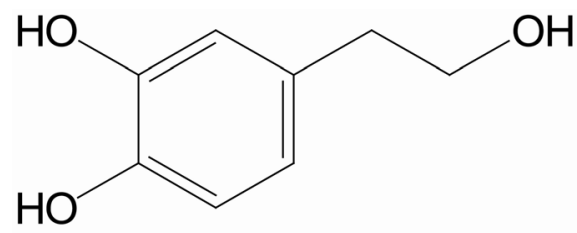

Figure 1. Molecular structure of hydroxytyrosol.

The aim of the present study is to investigate the protective effect of hydroxytyrosol against the oxidative stress-induced reduction in skeletal muscle force production. $\mathrm{H}_{2} \mathrm{O}_{2}$ is used to induce oxidative stress in skeletal muscle. In the present study we use the in vitro rat diaphragm preparation, which is a wellestablished model to study skeletal muscle function in vitro (15). 


\section{MATERIALS AND METHODS}

\section{Chemicals}

Hydroxytyrosol was obtained from Cayman Chemical Company, Ann Arbor, $\mathrm{MI}$, USA. Mannitol, 2-deoxy-D-ribose, $\mathrm{H}_{2} \mathrm{O}_{2}$ and ferric chloride hexahydrate were purchased from Sigma-Aldrich, St Louis, USA. Ascorbate was obtained from Merck, Darmstadt, Germany. All other chemicals were of analytical grade purity.

\section{Isolated diaphragm muscle strips}

The experimental protocol was approved by the Ethics Committee for Animal Experiments of the University of Maastricht before the start of the study. Male Lewis rats (12-14 wk old) were decapitated, the diaphragm was rapidly excised, and small strips (around $2 \mathrm{~mm}$ width and $1 \mathrm{~cm}$ long) were mounted in thermostated organ baths containing Krebs buffer $(\mathrm{pH} 7.4)$ gassed with $95 \% \mathrm{O}_{2}-5 \%$ $\mathrm{CO}_{2}$. Each diaphragm strip was connected vertically to an isometric transducer. The composition of the Krebs buffer was (mM): $\mathrm{NaCl}$ (117.5), $\mathrm{KCl}(5.6), \mathrm{MgSO}_{4}$ (1.18), $\mathrm{CaCl}_{2}$ (2.5), $\mathrm{NaH}_{2} \mathrm{PO}_{4}$ (1.28), $\mathrm{NaHCO}_{3}$ (25) and glucose (5.5). Field stimulation was created along the entire length of each muscle strip with platinum electrodes.

At the beginning of the experiment, the strips were washed during $45 \mathrm{~min}$ at room temperature. Fifteen minutes before the start of the experiment, the temperature of the baths was increased to $37^{\circ} \mathrm{C}$. Each strip was first adjusted to its optimal length $\left(\mathrm{L}_{\mathrm{o}}\right)$ using twitch contractions. After the adjustment to $\mathrm{L}_{\mathrm{o}}$, the strips were washed during $15 \mathrm{~min}$.

\section{Measurement of contractile function}

Firstly, the response of the diaphragm strips to single pulse stimulation, i.e. single twitch, was determined. Thereafter, the response of the diaphragm muscle strips to increasing stimulus frequencies was assessed by the application of 10, 20, 33, 50 and $100 \mathrm{~Hz}$-pulses applied in 500 ms trains. A 2-min recovery period was used between contractions. After the completion of this shortterm stimulation protocol, a fatigue protocol was applied. During the fatigue protocol, the strips were stimulated for $6 \mathrm{~min}$ at $5 \mathrm{~Hz}$. The pulse duration was $10 \mathrm{~ms}$ during the entire experiment. Subsequently, the strips were washed during 15 min. 


\section{Incubations}

When hydroxytyrosol was used, it was added 5 minutes before $\mathrm{H}_{2} \mathrm{O}_{2}$ was added. Hydroxytyrosol remained in the organ bath during the incubation with $\mathrm{H}_{2} \mathrm{O}_{2}$. In some experiments, the muscle strips were incubated only with hydroxytyrosol, i.e. without the subsequent addition of $\mathrm{H}_{2} \mathrm{O}_{2}$. In control experiments neither $\mathrm{H}_{2} \mathrm{O}_{2}$, nor hydroxytyrosol was added to the organ baths.

Ten min after the addition of $\mathrm{H}_{2} \mathrm{O}_{2}$, the strips were washed for 15 min. After washing the diaphragm, the contractile function of the muscle strips (short-term stimulation and fatigue) was determined for a second time as described above. In this way, each muscle bundle served as its own control (Figure 2).

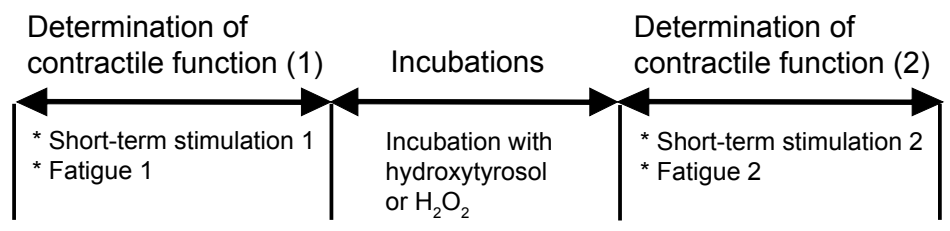

Figure 2. Schematic illustration of the various procedures of the study in time. Details on the protocol are given in the Materials and Methods section.

\section{Calculation of the effects of hydroxytyrosol and $\mathrm{H}_{2} \mathrm{O}_{2}$ on contractile function}

The contractile function of the muscle strips was determined before ('stimulation 1') and after incubation ('stimulation 2') with hydroxytyrosol and $\mathrm{H}_{2} \mathrm{O}_{2}$ (Figure 2). The force production of the muscle strips after incubation with $\mathrm{H}_{2} \mathrm{O}_{2}$ and hydroxytyrosol was compared with the initial force production before the incubations ('stimulation 1'). The effects of $\mathrm{H}_{2} \mathrm{O}_{2}$ and hydroxytyrosol on force production were expressed as the percentage of the initial force production.

\section{$\mathrm{H}_{2} \mathrm{O}_{2}$ scavenging}

Scavenging of $\mathrm{H}_{2} \mathrm{O}_{2}$ was measured by directly measuring the hydroxytyrosol concentration after adding $1 \mathrm{mM} \mathrm{H}_{2} \mathrm{O}_{2}$ to a $100 \mu \mathrm{M}$ solution of the hydroxyty-

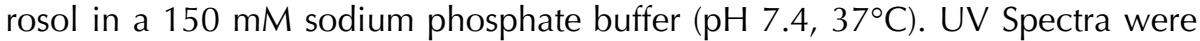
recorded before and during 30 min after the addition of $\mathrm{H}_{2} \mathrm{O}_{2}$.

\section{$\mathrm{OH}^{\bullet}$ scavenging}

Scavenging of $\mathrm{OH}^{\bullet}$ was determined by the deoxyribose method according to Haenen et al. (16). 


\section{Statistics}

Values are means \pm SEM. Differences in the response of the muscle strips were statistically analysed by Mann Whitney $\mathrm{U} . \mathrm{P}<0.05$ was considered statistically significant.

Control experiments (neither $\mathrm{H}_{2} \mathrm{O}_{2}$, nor hydroxytyrosol present) were performed on muscle strips obtained from five rats. The experiments with $\mathrm{H}_{2} \mathrm{O}_{2}$ (no hydroxytyrosol present), were performed on muscle strips of eight $\left(1 \mathrm{mM} \mathrm{H}_{2} \mathrm{O}_{2}\right.$ ) and two $\left(2 \mathrm{mM} \mathrm{H}_{2} \mathrm{O}_{2}\right)$ rats. The experiments with hydroxytyrosol were performed on muscle strips of three rats. Muscle strips of respectively one and two rats were used when 100 and $300 \mu \mathrm{M}$ hydroxytyrosol was incubated before the addition of $\mathrm{H}_{2} \mathrm{O}_{2}$. For the experiments in which muscle strips of two rats were used, the data are presented as mean \pm half range.

\section{RESULTS}

\section{Short-term electrical muscle stimulation}

Firstly, the concentration-dependent effect of $\mathrm{H}_{2} \mathrm{O}_{2}$ on force production was investigated. The force production of the muscle strips was determined before ('short-term stimulation 1') and after ('short-term stimulation 2') the incubation with $\mathrm{H}_{2} \mathrm{O}_{2}$ and hydroxytyrosol.

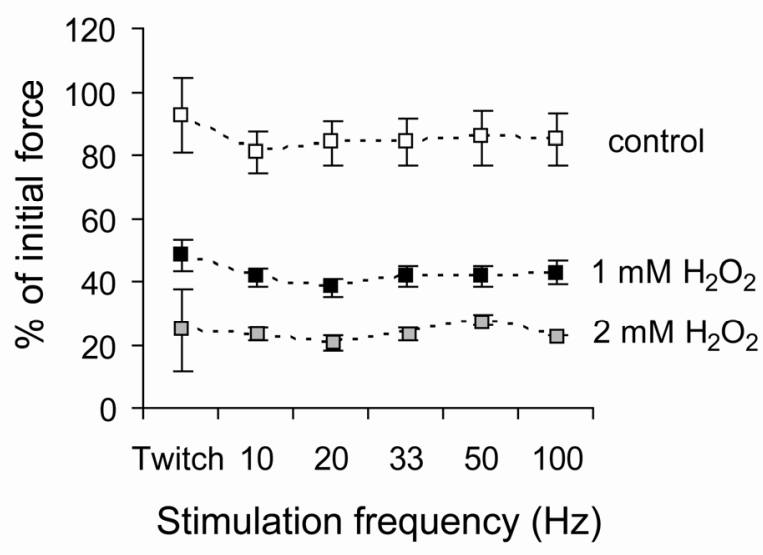

Figure 3. Effect of $1 \mathrm{mM}$ (black squares) and $2 \mathrm{mM}$ (grey squares) $\mathrm{H}_{2} \mathrm{O}_{2}$ on force production of diaphragm strips during short-term stimulation, compared with control (white squares). Values are the percentage of the initial force production during stimulation protocol 1. 
In the control experiment without $\mathrm{H}_{2} \mathrm{O}_{2}$, force production during 'short-term stimulation $2^{\prime}$ was approximately $80 \%$ of that of 'short-term stimulation $1^{\prime}$ ', at both low and high stimulation frequencies (Figure 3, white squares).

Incubation of the diaphragm muscle strips with $\mathrm{H}_{2} \mathrm{O}_{2}$ resulted in a considerable decrease in force production at a wide range of stimulation frequencies (Figure 3). Exposure of the muscle strips to $\mathrm{H}_{2} \mathrm{O}_{2}$ resulted in a $50 \%\left(1 \mathrm{mM} \mathrm{H}_{2} \mathrm{O}_{2}\right)$ to $75 \%\left(2 \mathrm{mM} \mathrm{H}_{2} \mathrm{O}_{2}\right)$ decrease in force production, compared to that of control muscle strips that were not exposed to hydroxytyrosol or $\mathrm{H}_{2} \mathrm{O}_{2}(\mathrm{P}<0.05$; Figure 3 and Figure 4). Based on these results, it was decided to use a concentration of $1 \mathrm{mM} \mathrm{H}_{2} \mathrm{O}_{2}$ to study the protective effect of hydroxytyrosol, as this concentration gave a substantial but submaximal effect.

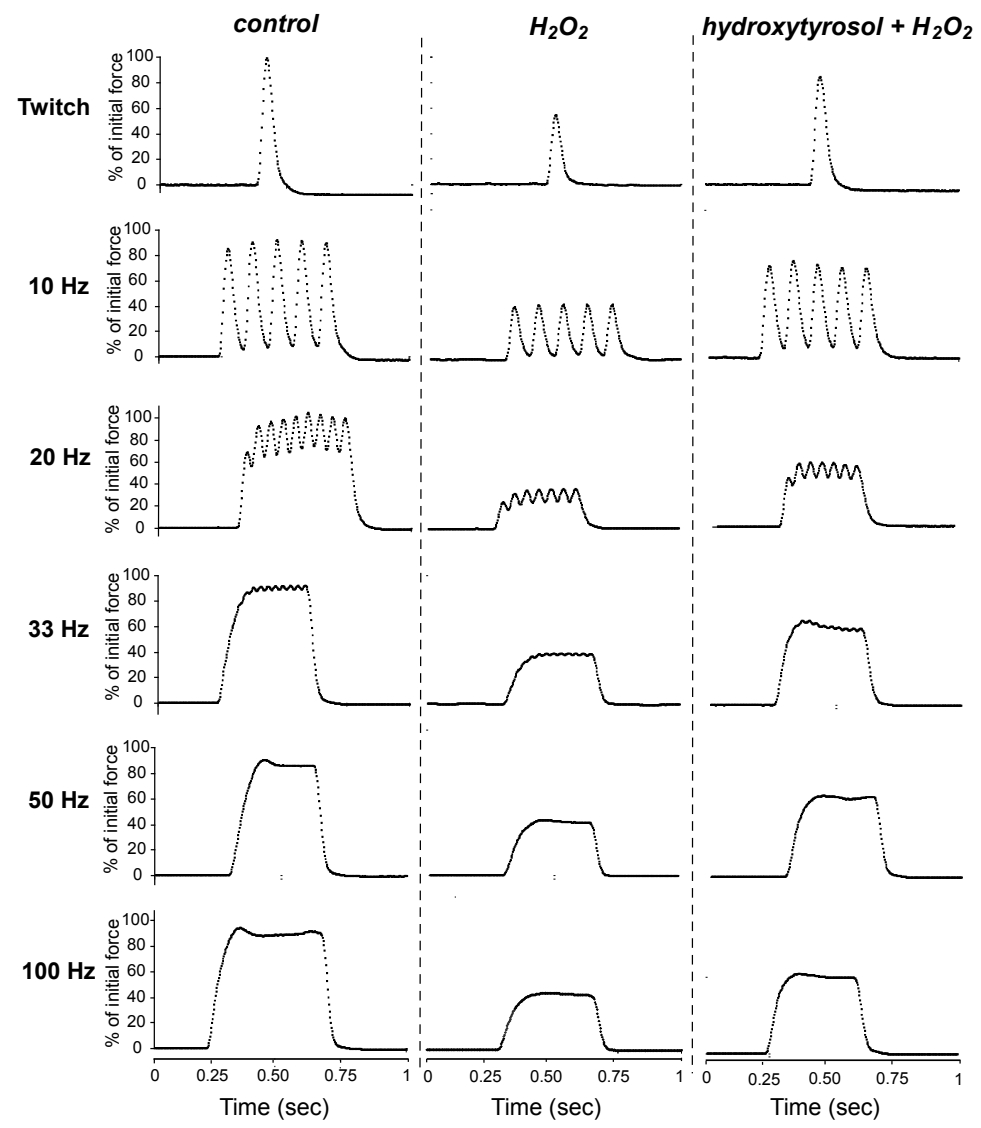

Figure 4. Effect of $\mathrm{H}_{2} \mathrm{O}_{2}(1 \mathrm{mM})$ on force production of diaphragm strips during short-term stimulation, compared to control. The protective effect of $50 \mu \mathrm{M}$ hydroxytyrosol is also shown. Diaphragm strips were stimulated before and after the addition of $\mathrm{H}_{2} \mathrm{O}_{2}$ and hydroxytyrosol. Values are the percentage of the initial force production during stimulation protocol 1 . Representative experiments are shown. 
Hydroxytyrosol protected the muscle strips against the $\mathrm{H}_{2} \mathrm{O}_{2}$-dependent decrease in force production (Figure 4 and Figure 5). Even a relatively low concentration of $25 \mu \mathrm{M}$ hydroxytyrosol significantly protected against $1 \mathrm{mM}$ $\mathrm{H}_{2} \mathrm{O}_{2}(\mathrm{P}<0.05)$ (Figure 5A). The dose-dependency of the protective effect of hydroxytyrosol against the $\mathrm{H}_{2} \mathrm{O}_{2}$-induced decline in force production during short-term $50 \mathrm{~Hz}$ stimulation is depicted in Figure 5B. A concentration of 300 $\mu \mathrm{M}$ hydroxytyrosol resulted in a protective effect of approximately $75 \%$ (Figure $5 B)$.

Hydroxytyrosol alone, in a concentration of $300 \mu \mathrm{M}$, did not affect muscle contractile function in response to short-term electrical stimulation (data not shown).
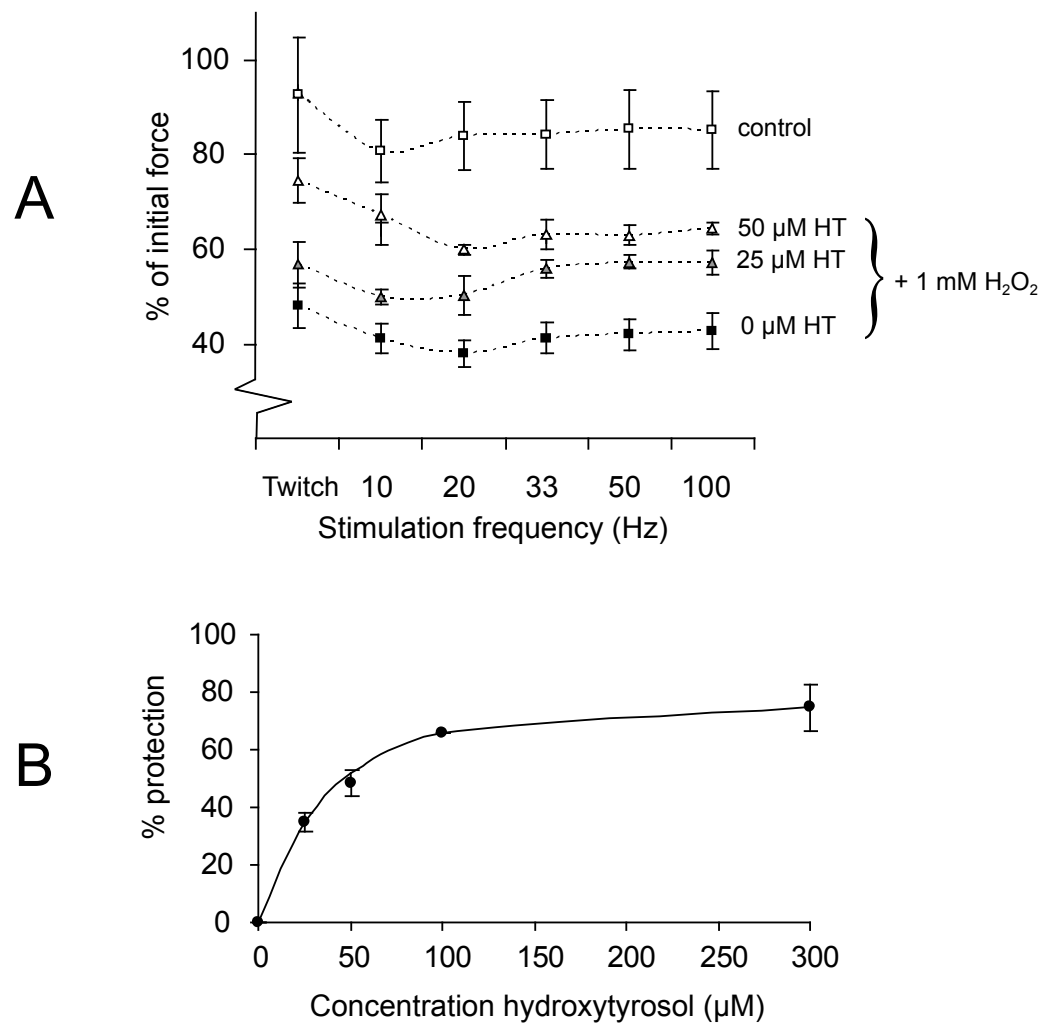

Figure 5. Protective effect of hydroxytyrosol (white and grey triangles) against the $\mathrm{H}_{2} \mathrm{O}_{2}(1 \mathrm{mM}$, black squares) induced decrease in force production during short-term stimulation, compared to control (white squares) (panel A). Different stimulation frequencies are shown. Values are the percentage of the initial force production during stimulation protocol 1. The concentrationdependent protection of hydroxytyrosol (HT) against the $\mathrm{H}_{2} \mathrm{O}_{2}(1 \mathrm{mM})$ induced decline in force production at a stimulation frequency of $50 \mathrm{~Hz}$ is shown in panel $\mathrm{B}$. 


\section{Fatigue}

During the first two min of long-term electrical muscle stimulation a rapid decline in force production was observed. Subsequently, the force production gradually decreased during the last 4 min of the fatigue protocol (Fatigue 1; Figure 6 and Figure 7). When the muscle strips were once again stimulated for 6 min (Fatigue 2), force production was approximately 20\% lower compared to Fatigue 1. In these control experiments the diaphragm muscle strips were not incubated with hydroxytyrosol or $\mathrm{H}_{2} \mathrm{O}_{2}$ (Figure 6A and Figure 7).

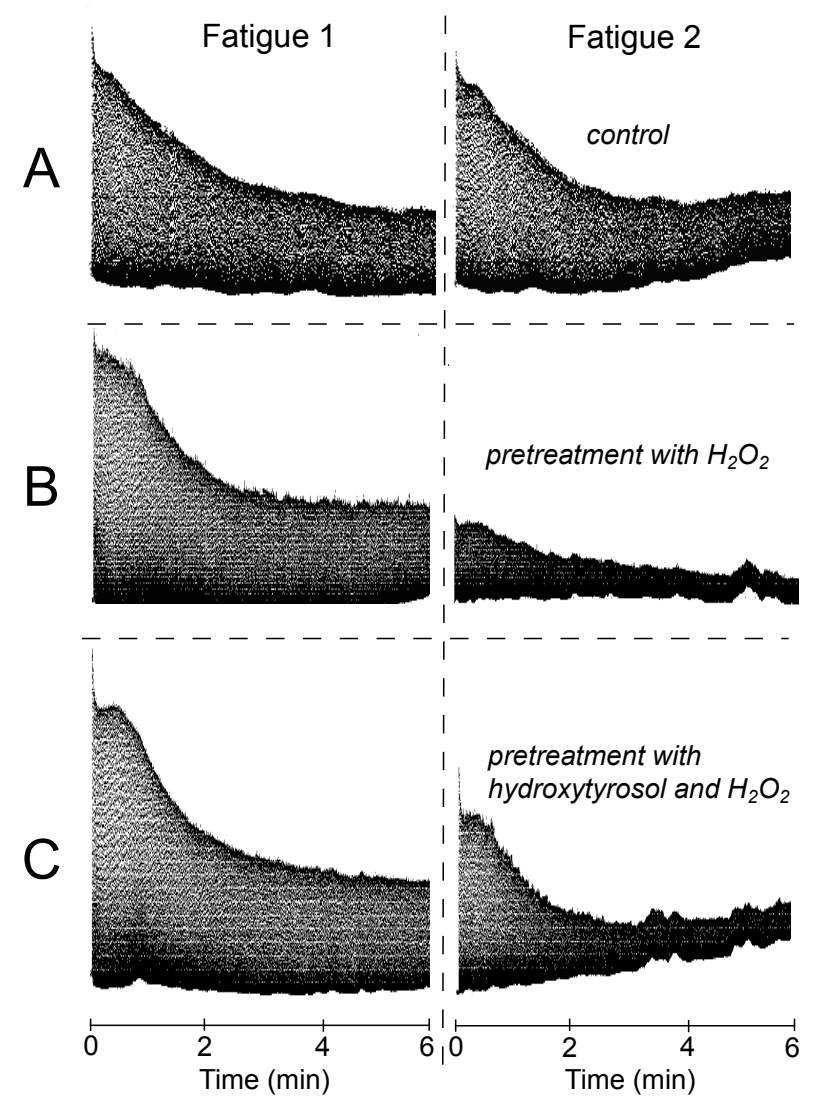

Figure 6. Effect of $\mathrm{H}_{2} \mathrm{O}_{2}(1 \mathrm{mM})$ on force production of diaphragm strips during long-term stimulation, compared to control. The protective effect of $50 \mu \mathrm{M}$ hydroxytyrosol is also shown. Representative recorder traces are shown.

$\mathrm{H}_{2} \mathrm{O}_{2}$ drastically impaired the initial and long term force production during the 6-min stimulation protocol (Fatigue 2; Figure 6B and 7). Hydroxytyrosol protected the muscle strips against the $\mathrm{H}_{2} \mathrm{O}_{2}$-induced impairment in force 
production, especially during the initial stage of fatigue (Fatigue 2; Figure 6C and Figure 7). Hydroxytyrosol alone, in a concentration of $300 \mu \mathrm{M}$, did not affect diaphragm force production during the long-term fatigue protocol (data not shown).

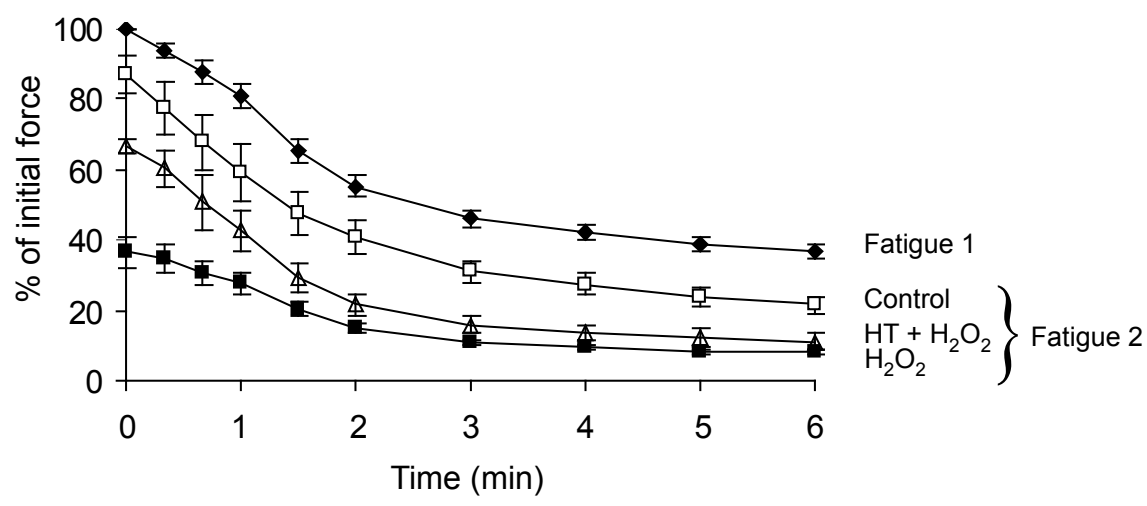

Figure 7. Effect of long-term stimulation on force production of diaphragm strips (black quadrangle). Effect of $\mathrm{H}_{2} \mathrm{O}_{2}(1 \mathrm{mM})$ (black squares) on force production of diaphragm strips during longterm stimulation, compared to control (white squares). The protective effect of $50 \mu \mathrm{M}$ hydroxytyrosol $(\mathrm{HT})$ is also shown (white triangles). Values are the percentage of the initial force production.

\section{$\mathrm{H}_{2} \mathrm{O}_{2}$ and $\mathrm{OH}^{\bullet}$ scavenging}

Hydroxytyrosol did not show any $\mathrm{H}_{2} \mathrm{O}_{2}$ scavenging activity. In contrast, hydroxytyrosol was found to be a potent $\mathrm{OH}^{*}$ scavenger. The $\mathrm{OH}^{*}$ scavenging activity of hydroxytyrosol (second order rate constant, $\mathrm{k}_{\mathrm{s}}=25.1 \cdot 10^{9} \mathrm{M}^{-1} \mathrm{~s}^{-1}$ ) was nearly seven times higher than the activity of mannitol, a well known $\mathrm{OH}^{*}$ scavenger $\left(k_{\mathrm{s}}=3.4 \cdot 10^{9} \mathrm{M}^{-1} \mathrm{~s}^{-1}\right)$.

\section{DISCUSSION}

The production of ROS is significantly enhanced during skeletal muscle contractile activity. When the production of ROS exceeds the protective effect provided by antioxidants, oxidative damage will occur $(2,3)$. In skeletal muscle, this will lead to reduced force production. Therefore, increasing the antioxidant capacity of skeletal muscle cells seems to be valuable to reduce oxidative muscle damage and preserve the contractile function. 
A number of studies have investigated the effect of exogenous antioxidants on skeletal muscle contractile function. For example, $\mathrm{N}$-acetylcysteine (NAC) has been shown to attenuate fatigue in both rat (17) and rabbit (18) diaphragms. Moreover, NAC has been shown to delay fatigue in several human intervention studies (19-22). In contrast, incubation of rat diaphragm with NAC depressed the contractility during short-term stimulation at low frequencies. Similar to $\mathrm{NAC}$, vitamin $\mathrm{E}$ also reduced the force production of isolated diaphragm strips during short-term stimulation at low stimulation frequencies (15). In contrast to NAC, vitamin E did not affect fatigue during long-term stimulation. However, trolox, a water-soluble vitamin $\mathrm{E}$ analog, attenuated the mechanical ventilationinduced impairment in contractile function of rat diaphragm (23). The antioxidant lipoic acid did not affect skeletal muscle contractile function (15). Although the effects of various antioxidants on contractile performance are not unequivocal, the common view is that antioxidants might protect skeletal muscle force production during exercise.

In the present study it was shown that hydroxytyrosol efficiently protected against the $\mathrm{H}_{2} \mathrm{O}_{2}$-induced reduction in force production especially during the initial stage of fatigue. Moreover, hydroxytyrosol also protected the muscle strips against the $\mathrm{H}_{2} \mathrm{O}_{2}$-induced reduction in force production during short-term stimulation, at a wide range of stimulation frequencies.

A relatively low concentration $(25 \mu \mathrm{M})$ of hydroxytyrosol protected significantly against a forty-fold higher concentration of $\mathrm{H}_{2} \mathrm{O}_{2}(1 \mathrm{mM})$. Moreover, hydroxytyrosol displayed no $\mathrm{H}_{2} \mathrm{O}_{2}$ scavenging activity. This indicates that $\mathrm{H}_{2} \mathrm{O}_{2}$ is converted to another reactive species that subsequently impairs skeletal muscle contractile function, such as the highly reactive $\mathrm{OH}^{*}$. Because of its extreme reactivity, $\mathrm{OH}^{*}$ acts in an indiscriminate way. It is able to destruct any cellular component. This can have far-reaching consequences. For example, $\mathrm{OH}^{*}$ can induce lipid peroxidation, leading to damage of cytoskeleton and membranes and, consequently, cell dysfunction. The excellent antioxidant efficacy of hydroxytyrosol is illustrated by its potent $\mathrm{OH}^{*}$ scavenging activity, which is nearly seven times higher than the activity of mannitol, a well known $\mathrm{OH}^{\cdot}$ scavenger.

Muscle contraction involves a chain of sequential reactions. Like any other cellular process, all of these reactions can be inhibited by radicals. One of these reactions will be relatively more susceptible to radical damage than the other reactions involved in muscle contraction. This reaction, referred to as 'the bottle neck', will become limiting in muscle contraction during oxidative stress (24). By breaking this weakest link, the chain of reactions leading to contraction is broken, thereby limiting force production and concomitant ROS production. Reid compared this self-limiting radical production with 'a-canary-in-the-coalmine' (24). A canary is relatively sensitive to toxic gasses. Therefore, miners used to take a canary into the mine as a biosensor. When the canary dropped 
dead, this signalled the miners to immediately evacuate the mine in order to avoid the same fate. The comparison, however, falls short as the 'sensor', i.e. the weakest link in muscle contraction, is an integral part of the chain of reactions that needs to be protected. Moreover, radicals have a very limited specificity and will also react with cell components other than the sensor. With regard to the indiscriminate action of radicals, the metaphor of 'a-bull-in-achina-store' seems to be more appropriate. The present study shows that the species that are involved are very reactive, comparable to that of $\mathrm{OH}^{\circ}$. Such a reactive species will destroy anything that comes in its way.

In line with 'the-canary-in-the-coal-mine' concept, Reid argued that the use of antioxidants to increase performance might backfire. Antioxidants were supposed to only reduce the sensitivity of the sensor. The trade-off of antioxidant supplementation would be better short-term performance at the expense of greater exercise-induced injury and longer recovery times (24). The sensor will relatively benefit the most from antioxidant supplementation. However, by antioxidant supplementation the other parts of the chain of reactions leading to muscle contraction are protected to the same degree as the sensor. Also after antioxidant supplementation the sensor remains the weakest link. After antioxidant supplementation, more exercise induced ROS have to be produced to inactivate the sensor, or, in other words, to break this weakest link. The high vulnerability of the weakest link relative to that of the other parts of the chain is not changed by antioxidant supplementation. This indicates that the theoretical trade-off for the use of antioxidants to increase performance is not plausible. In our opinion, by 'taming the bull', i.e. by inactivating radicals, antioxidants protect the entire 'china store'.

For an antioxidant to have beneficial health effects in vivo it is of course essential that it is bioavailable. Several studies in humans and rats indeed report that the uptake of hydroxytyrosol is good (25-28). Plasma concentrations of hydroxytyrosol after consumption of $25 \mathrm{ml}$ of extra virgin olive oil range from 50 to $160 \mathrm{nM}(29,30)$. The consumption of supplements containing a relatively high amount of hydroxytyrosol might lead to even higher plasma hydroxytyrosol concentrations.

The concentrations of hydroxytyrosol tested were, compared to the concentrations reached in vivo, relatively high. However, the concentration of $\mathrm{H}_{2} \mathrm{O}_{2}$ required to induce oxidative damage in the isolated diaphragm in a relatively short time span was also relatively high, i.e. $1 \mathrm{mM}$. The level of oxidative stress in the body is lower than that generated in the organ baths. Nevertheless, hydroxytyrosol was able to provide substantial protection against the relatively high concentration of $\mathrm{H}_{2} \mathrm{O}_{2}$. This indicates that hydroxytyrosol has the potency to protect against oxidative stress in skeletal muscle in vivo. Several studies have demonstrated that a real-life intake of hydroxytyrosol displays a physiological relevant antioxidant effect. For example, hydroxytyrosol protects against 
the oxidative modification of LDL (10). This shows that hydroxytyrosol behaves as an efficient antioxidant in vivo.

One of the problems in comparing the efficacy of hydroxytyrosol with that of antioxidants used in other studies is that the concentrations used of both antioxidant and oxidant and the applied study design are highly variable. Compared to the most frequent studied antioxidant in muscle concentration, i.e. NAC, hydroxytyrosol seems to have at least the same the same potency in the isolated diaphragm. Both hydroxytyrosol and NAC did reduce fatigue during long-term stimulation $(17,18)$. However, hydroxytyrosol, in contrast to NAC, did not reduce the force production of isolated diaphragm strips at low stimulation frequencies. It should be noted that the present study is - to our knowledge - the first study thus far on the effects of hydroxytyrosol on skeletal muscle force production and in vivo data on this subject are lacking.

In principle, hydroxytyrosol supplementation might have several applications. Firstly, hydroxytyrosol might mitigate exercise induced oxidative muscle damage $(3,4,31)$. Furthermore, hydroxytyrosol could theoretically be used as a performance-enhancing nutraceutical in certain conditions. For example, in several respiratory diseases, including chronic obstructive pulmonary disease (COPD), diaphragm force generation is impaired. Impaired respiratory muscle function causes ventilatory failure and exercise intolerance. In the aetiology of the impaired muscle performance, oxidative stress has been implicated (32). Hydroxytyrosol supplementation might protect the diaphragm, which would drastically improve the quality of life of these patients. The present study strengthens the rationale to further investigate the benefit of antioxidants such as hydroxytyrosol in these situations. 


\section{REFERENCES}

1. Reid MB, Khawli FA, Moody MR. Reactive oxygen in skeletal muscle. III. Contractility of unfatigued muscle. J Appl Physiol. 1993;75(3):1081-7.

2. Bast A, Haenen GR, Doelman CJ. Oxidants and antioxidants: state of the art. Am J Med. 1991;91(3C):2S-13S.

3. Vollaard NB, Shearman JP, Cooper CE. Exercise-induced oxidative stress:myths, realities and physiological relevance. Sports Med. 2005;35(12):1045-62.

4. Rietjens SJ, Beelen M, Koopman R, van Loon LJ, Bast A, Haenen GR. A single session of resistance exercise induces oxidative damage in untrained men. Med Sci Sports Exerc. 2007;39(12):2145-51.

5. Zissimopoulos S, Lai FA. Redox regulation of the ryanodine receptor/calcium release channel. Biochem Soc Trans. 2006;34(Pt 5):919-21.

6. Nagasawa T, Hatayama T, Watanabe $Y$, Tanaka M, Niisato Y, Kitts DD. Free radical-mediated effects on skeletal muscle protein in rats treated with Fe-nitrilotriacetate. Biochem Biophys Res Commun. 1997;231(1):37-41.

7. Coirault C, Guellich A, Barbry T, Samuel JL, Riou B, Lecarpentier Y. Oxidative stress of myosin contributes to skeletal muscle dysfunction in rats with chronic heart failure. Am J Physiol Heart Circ Physiol. 2007;292(2):H1009-17.

8. Kim NH, Jeong MS, Choi SY, Kang JH. Oxidative modification of cytochrome c by hydrogen peroxide. Mol Cells. 2006;22(2):220-7.

9. Balon TW, Yerneni KK. Redox regulation of skeletal muscle glucose transport. Med Sci Sports Exerc. 2001;33(3):382-5.

10. Rietjens SJ, Bast A, Haenen GR. New insights into controversies on the antioxidant potential of the olive oil antioxidant hydroxytyrosol. J Agric Food Chem. 2007;55(18):7609-14.

11. Rietjens SJ, Bast A, de Vente J, Haenen GR. The olive oil antioxidant hydroxytyrosol efficiently protects against the oxidative stress-induced impairment of the $\mathrm{NO}^{\bullet}$ response of isolated rat aorta. Am J Physiol Heart Circ Physiol. 2007;292(4):H1931-6.

12. Manna C, Galletti P, Cucciolla V, Moltedo O, Leone A, Zappia V. The protective effect of the olive oil polyphenol (3,4-dihydroxyphenyl)-ethanol counteracts reactive oxygen metaboliteinduced cytotoxicity in Caco-2 cells. J Nutr. 1997;127(2):286-92.

13. Goya L, Mateos R, Bravo L. Effect of the olive oil phenol hydroxytyrosol on human hepatoma HepG2 cells : Protection against oxidative stress induced by tert-butylhydroperoxide. Eur J Nutr. 2007;46(2):70-8.

14. Manna C, Galletti P, Cucciolla V, Montedoro G, Zappia V. Olive oil hydroxytyrosol protects human erythrocytes against oxidative damages. J Nutr Biochem. 1999;10:159-165.

15. Coombes JS, Powers SK, Rowell B, et al. Effects of vitamin E and alpha-lipoic acid on skeletal muscle contractile properties. J Appl Physiol. 2001;90(4):1424-30.

16. Haenen GR, Jansen FP, Bast A. The antioxidant properties of five O-(b-hydroxyethyl)-rutosides of the flavonoid mixture venoruton. Phlebology. 1993;suppl. 1:10-17.

17. Khawli FA, Reid MB. N-acetylcysteine depresses contractile function and inhibits fatigue of diaphragm in vitro. J Appl Physiol. 1994;77(1):317-24.

18. Shindoh C, DiMarco A, Thomas A, Manubay P, Supinski G. Effect of N-acetylcysteine on diaphragm fatigue. J Appl Physiol. 1990;68(5):2107-13.

19. Medved I, Brown MJ, Bjorksten AR, et al. N-acetylcysteine enhances muscle cysteine and glutathione availability and attenuates fatigue during prolonged exercise in endurance-trained individuals. J Appl Physiol. 2004;97(4):1477-85.

20. Matuszczak Y, Farid M, Jones J, et al. Effects of $\mathrm{N}$-acetylcysteine on glutathione oxidation and fatigue during handgrip exercise. Muscle Nerve. 2005;32(5):633-8.

21. Reid MB, Stokic DS, Koch SM, Khawli FA, Leis AA. N-acetylcysteine inhibits muscle fatigue in humans. J Clin Invest. 1994;94(6):2468-74. 
22. Travaline JM, Sudarshan S, Roy BG, Cordova F, Leyenson V, Criner GJ. Effect of Nacetylcysteine on human diaphragm strength and fatigability. Am J Respir Crit Care Med. 1997;156(5):1567-71.

23. Betters JL, Criswell DS, Shanely RA, et al. Trolox attenuates mechanical ventilation-induced diaphragmatic dysfunction and proteolysis. Am J Respir Crit Care Med. 2004;170(11):1179-84.

24. Reid MB. Free radicals and muscle fatigue: of ROS, canaries, and the IOC. Free Radic Biol Med. 2008;44(2):169-79.

25. Visioli F, Caruso D, Plasmati E, et al. Hydroxytyrosol, as a component of olive mill waste water, is dose- dependently absorbed and increases the antioxidant capacity of rat plasma. Free Radic Res. 2001;34(3):301-5.

26. Visioli F, Galli C, Bornet F, et al. Olive oil phenolics are dose-dependently absorbed in humans. FEBS Lett. 2000;468(2-3):159-60.

27. Vissers MN, Zock PL, Roodenburg AJ, Leenen R, Katan MB. Olive oil phenols are absorbed in humans. J Nutr. 2002;132(3):409-17.

28. Tuck KL, Freeman MP, Hayball PJ, Stretch GL, Stupans I. The in vivo fate of hydroxytyrosol and tyrosol, antioxidant phenolic constituents of olive oil, after intravenous and oral dosing of labeled compounds to rats. J Nutr. 2001;131(7):1993-6.

29. Weinbrenner $T$, Fito $M$, de la Torre $R$, et al. Olive oils high in phenolic compounds modulate oxidative/antioxidative status in men. J Nutr. 2004;134(9):2314-21.

30. Miro Casas E, Covas MI, Farre M, et al. Hydroxytyrosol disposition in humans. Clin Chem. 2003;49(6 Pt 1):945-52.

31. Bloomer RJ, Goldfarb AH. Anaerobic exercise and oxidative stress: a review. Can J Appl Physiol. 2004;29(3):245-63.

32. Gosker HR, Wouters EF, van der Vusse GJ, Schols AM. Skeletal muscle dysfunction in chronic obstructive pulmonary disease and chronic heart failure: underlying mechanisms and therapy perspectives. Am J Clin Nutr. 2000;71(5):1033-47. 



\section{CHAPTER 6}

The olive antioxidant hydroxytyrosol boosts the endogenous antioxidant defence and attenuates the rise in plasma lactate during resistance type exercise

S.J. Rietjens, M. Beelen, L.J.C. van Loon, A. Bast and G.R.M.M. Haenen

Submitted

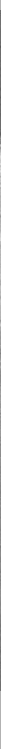




\section{ABSTRACT}

During exercise, the production of reactive oxygen and nitrogen species is considerably enhanced. The aim of the present study was to investigate the effects of the olive antioxidant hydroxytyrosol on antioxidant capacity, oxidative damage, inflammation, and plasma lactate levels during strenuous exercise. In a cross-over design, 7 untrained healthy men were randomly assigned to supplementation with hydroxytyrosol-rich olive extract or placebo. Muscle biopsies, urine, and blood samples were collected before and after a single bout of resistance type exercise. Supplementation with hydroxytyrosol (twice $200 \mathrm{mg}$ ) resulted in a considerable increase in plasma total antioxidant capacity (TEAC). Moreover, the glutathione defence system in skeletal muscle tissue was up-regulated by hydroxytyrosol, as shown by an elevation in glutathione (GSH) levels and increased glutathione reductase activity after exercise. Urinary isoprostane levels were not altered by hydroxytyrosol. Inflammatory markers were neither affected by exercise, nor by hydroxytyrosol supplementation. Interestingly, hydroxytyrosol attenuated the rise in circulating plasma lactate levels during exercise. 


\section{INTRODUCTION}

Exercise magnifies the production of reactive oxygen species (ROS) in skeletal muscle tissue. Potential sources for ROS production during exercise are mitochondria, plasma membrane NADPH oxidases, phospholipase $A_{2}$ dependent processes, and ischemia reperfusion (1-4). Radical production may lead to tissue injury. Moreover, ROS trigger the activation of pro-inflammatory pathways such as Nuclear Factor kappa B (NF-kB) and the invasion of the injured tissue with phagocytic cells. These pathways further promote the production of ROS in a feed forward process.

Fortunately, the body has an elaborate defence system of antioxidants that provides protection against ROS. A major part of the defence against ROS is made up by alimentary antioxidants. In spite of the intricate antioxidant network, a large number of human studies have shown that exercise induces lipid peroxidation, as demonstrated by an increase in thiobarbituric acid reactive substances (TBARS), isoprostanes and exhaled pentane (5-14). The increase in these biomarkers indicates that endogenous antioxidants do not adequately cope with increased radical production during exercise. This provides the rationale for the supplementation of antioxidants during exercise. Several studies on the effects of increased antioxidant intake on exerciseinduced skeletal muscle injury and inflammation have been performed. However, these studies are ambiguous, showing inconsistent results $(15,16)$.

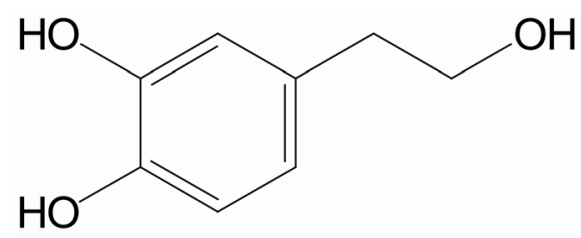

Figure 1. Molecular structure of hydroxytyrosol.

In the present study, the effect of the olive oil antioxidant hydroxytyrosol (Figure 1) during exercise was studied. Hydroxytyrosol was selected because it is a versatile antioxidant, showing strong antioxidant activity in both in vitro and in vivo studies (17-24). Furthermore, it has been recently found that hydroxytyrosol efficiently protects against oxidative stress-induced reduction in force production of skeletal muscle in vitro (chapter 5 of the thesis). In the present study, we investigated the effects of hydroxytyrosol supplementation on antioxidant capacity, oxidative damage, inflammation, and lactate levels in untrained, healthy volunteers during a single session of resistance type exercise. 


\section{METHODS}

For the present study on the effect of hydroxytyrosol on antioxidant capacity, oxidative damage, inflammation, and lactate levels, we used the resistance exercise model as described in chapter 4 of the thesis. The placebo data of the study are described in chapter 4 of the thesis.

\section{Subjects}

Eight healthy male volunteers with no history of participating in any regular exercise program were recruited for the present study. One of the subjects was excluded from the study because he could not comply with the exercise protocol and had to stop before all the repetitions were performed. Subjects' characteristics are shown in Table 1.

Table 1. Subjects' characteristics

\begin{tabular}{lc}
\hline & Mean \pm SEM \\
\hline Age $(\mathrm{yrs})$ & $22.4 \pm 2.0$ \\
Body mass $(\mathrm{kg})$ & $74.4 \pm 3.3$ \\
Height $(\mathrm{m})$ & $1.82 \pm 0.03$ \\
BMI $\left(\mathrm{kg} \cdot \mathrm{m}^{-2}\right)$ & $22.3 \pm 0.8$ \\
\hline
\end{tabular}

Values are expressed as mean \pm SEM.

All subjects were informed on the nature and possible risks of the experimental procedures before their written informed consent was obtained. This study has been approved by the Medical Ethical Review Board of the Academic Hospital, Maastricht, the Netherlands.

\section{Pretesting}

Two weeks before the experimental trial, the subjects performed a short exercise session to become familiarized with the exercise protocol and the equipment. Proper weightlifting technique was demonstrated and practiced for each of the two lower-limb exercises (leg press and leg extension). Subsequently, maximum strength was estimated using a standard multiple repetitions testing procedure (25). After warming up, the load was gradually increased, and the number of successful lifts until failure was determined. A 5-min resting period between subsequent attempts was allowed.

In a second session, at least one week after the first session and at least one week before the experimental trial, subjects' one repetition maximum (1RM) for each of the two lower-limb exercises was determined (26). After warming up, the load was set at $90 \%$ of the estimated $1 \mathrm{RM}$ and was increased after each successful lift until failure. Thereafter, the load was increased by $2.5-5 \%$ and 
the successful lifts until failure were determined. A 5-min resting period between successive attempts was allowed. A repetition was valid if the subject was able to complete the entire lift in a controlled manner without assistance. $1 \mathrm{RM}$ was typically reached within three attempts.

Mean $1 \mathrm{RM}$ measured during pre-testing was $117 \pm 6 \mathrm{~kg}$ on the leg extension and $195 \pm 11 \mathrm{~kg}$ on the horizontal leg press. The starting workload applied during resistance exercise averaged $88 \pm 5 \mathrm{~kg}$ and $146 \pm 8 \mathrm{~kg}$ (75\% of 1RM) for the leg extension and leg press, respectively.

\section{Standardization of diet and activity prior to testing}

Subjects were instructed not to consume any products rich in antioxidants, such as fruit juices, chocolate, olive oil, wine, or antioxidant supplements during three days before testing, on the test day itself, and on the day after the test day until $24 \mathrm{~h}$ after exercise. The low-antioxidant diet was chosen firstly to minimize a potential protective effect of high antioxidant intake and, secondly, to reduce variation in the baseline level of antioxidants, such as vitamin $\mathrm{E}$ and vitamin C. The volunteers consumed a carbohydrate-rich noodle dish, containing approximately $10 \mathrm{~g}$ of the vegetable leek, at $8.00 \mathrm{pm}$ in the evening before the test day. Subjects were asked to record their food intake for three days before testing, on the test day itself, and on the day after the test day until $24 \mathrm{~h}$ after exercise. Food choices and quantities were recorded. By reviewing the recorded food intake, it was verified that the subjects' diet complied with the instructions. The volunteers were instructed to refrain from any sort of heavy physical exercise during the entire period, except for the resistance exercise session.

\section{Hydroxytyrosol supplementation}

The volunteers were asked to participate in the study on two separate occasions. Subjects consumed, in a randomized order, a hydroxytyrosol-rich olive extract or a placebo. The study was blinded for both the volunteers as well as the investigators. The olive extract (kindly provided by DSM, Delft, the Netherlands), providing $200 \mathrm{mg}$ hydroxytyrosol per dose, was diluted in $150 \mathrm{ml}$ drinking water, directly prior to ingestion. The olive extract or the placebo was supplemented twice: the evening before the test day at $8.00 \mathrm{pm}$ and on the test day itself at $8.30 \mathrm{am}(\mathrm{t}=-30 \mathrm{~min})$. The placebo had no antioxidant capacity, as determined by the trolox equivalent antioxidant capacity (TEAC) assay. The hydroxytyrosol supplement and the placebo were similar in taste and colour. 


\section{Experimental trial}

The subjects arrived at the laboratory at $8.00 \mathrm{am}$, in an overnight fasted state. Then, they performed a 5-min low-intensity warm up using a Stairmaster (Jimsa Benelux BV, Rotterdam, the Netherlands). Thereafter, the resistance exercise session targeted the legs, with eight sets of ten repetitions on the horizontal leg press machine (Technogym BV, Rotterdam, the Netherlands) and eight sets of ten repetitions on the leg extension machine (Technogym). The starting workload applied during resistance exercise session was $75 \%$ of the individual 1 RM for both the leg press and leg extension with 2-min rest intervals between sets. In total, the exercise regime required approximately $40 \mathrm{~min}$ to complete. This resistance exercise protocol has been applied previously and is routinely used as a resistance type exercise session in our institute $(25,26)$.

If subjects could not finish all ten repetitions at full weight, this was reduced to $65 \%$ or $55 \%$ of the individual 1 RM. Only one of the evaluated volunteers was able to finish the entire protocol at $75 \%$. The average intensity level of the exercise regime was $70 \%$. All subjects were verbally encouraged during the test to complete the entire protocol.

\section{Blood sampling}

A Teflon catheter was inserted in an antecubital vein for venous blood sampling. Blood was collected before the intake of hydroxytyrosol or placebo $(t=-$ $30 \mathrm{~min})$, just before the start of the exercise $(t=0)$, during exercise ( $t=20 \mathrm{~min})$, two min after cessation of exercise $(t=43 \mathrm{~min})$, and during subsequent recovery ( $\mathrm{t}=60 \mathrm{~min}, 90 \mathrm{~min}, 120 \mathrm{~min}, 150 \mathrm{~min}, 180 \mathrm{~min}$, and $24 \mathrm{~h}$ after exercise). Blood samples were collected in tubes containing heparin and placed on ice. Blood was aliquoted for the vitamin $\mathrm{C}$ and reduced glutathione (GSH) and glutathione disulphide (GSSG) analysis. To preserve the samples, 5\% trichloroacetic acid (TCA) was added to the former, and $1.3 \%$ sulphosalicylic acid (SSA) was added to the latter. After centrifugation at $1000 \cdot \mathrm{g}$ at $4{ }^{\circ} \mathrm{C}$ for $5 \mathrm{~min}$, aliquots of plasma were stored at $-80^{\circ} \mathrm{C}$ until analysis.

\section{Blood analyses}

Plasma lactate concentrations were analysed spectrophotometrically $(340 \mathrm{~nm})$, measuring $\mathrm{NADH}$ formation using lactate dehydrogenase (LDH) (27). The increase in lactate concentration above baseline was determined and the area under the curve (AUC) of this increase in time was calculated using the trapezoidal rule (28). The half-life $\left(\mathrm{t}_{1 / 2}\right)$ of lactate after exercise was calculated by plotting the natural logarithm of the increase in lactate against time. This gave a 
straight line, showing that the kinetics of lactate follow first order kinetics. The slope of the straight line obtained gives the elimination rate constant $k$.

The total antioxidant capacity (TEAC) was determined in plasma that was deproteinized with a final concentration of 5\% TCA (29). The samples were incubated with the ABTS radical solution for $5 \mathrm{~min}$ and subsequently the absorbance at $734 \mathrm{~nm}$ was measured. The TEAC is expressed as $\mu \mathrm{M}$ trolox equivalents.

Uric acid was determined in plasma that was deproteinized with a final concentration of $5 \% \mathrm{TCA}$, using HPLC. A Hypersil BDS C-18 end-capped column, $125 \times 4 \mathrm{~mm}$, particle size $5 \mu \mathrm{m}$ (Agilent, Palo Alto, CA, USA), was used, with a mobile phase of $0.1 \%$ trifluoroacetic acid $(\mathrm{v} / \mathrm{v})$ in water. UV detection was performed at $292 \mathrm{~nm}$. The relative contribution of uric acid to the TEAC is calculated using the TEAC value of 1 for uric acid (29).

Hydroxytyrosol and homovanillic alcohol were determined in plasma that was deproteinized with a final concentration of $3 \%$ perchloric acid (PCA) using HPLC. The system (Agilent, Palo Alto, CA, USA) consisted of an Alltima C18 column, $150 \times 3 \mathrm{~mm}$, particle size $5 \mu \mathrm{m}$ (Alltech, Deerfield, USA) with a mobile phase of $3 \%$ acetonitrile $(\mathrm{v} / \mathrm{v})$ and $0.1 \%$ trifluoric acid $(\mathrm{v} / \mathrm{v})$ in MilliQ. Diode array $(280 \mathrm{~nm})$ and fluorescence detection (excitation $281 \mathrm{~nm}$, emission $316 \mathrm{~nm}$ ) were used. Plasma samples were subjected to acidic hydrolysis to detect hydroxytyrosol metabolites, e.g. glucuronide or sulphate conjugates.

Vitamin C was determined in plasma by HPLC using the following method. Vitamin $\mathrm{C}$ was oxidized by ascorbate oxidase to dehydroascorbate. The latter reacted with o-phenylene diamine to its quinoxaline derivative. This derivative was quantified on the same system used for uric acid with a mobile phase of $0.08 \mathrm{M}$ phosphate buffer $(\mathrm{pH} 7.8)$ and methanol, with fluorimetric detection (355-425 nm).

Vitamin E was extracted using hexane after adding vitamin E nicotinate as internal standard. HPLC analysis was performed on the same system used for uric acid with a mobile phase of methanol. UV detection was used $(295 \mathrm{~nm})$.

The erythrocyte non-protein thiol concentration, representing mainly GSH, was measured using 5,5'-dithiobis 2-nitrobenzoic acid (DTNB). The erythrocyte GSSG concentration was measured using 2-vinylpyridine pre-treatment using the recycling method (30).

\section{NF-KB activity in peripheral blood mononuclear cells (PBMCs)}

PBMCs were isolated from the blood samples (collected into leucosep tubes at $\mathrm{t}=0,43 \mathrm{~min}, 90 \mathrm{~min}$, and $24 \mathrm{~h}$ after exercise) using Lymphoprep (Bio-Connect, Huissen, the Netherlands). Nuclear extracts were immediately prepared and stored at $-80^{\circ} \mathrm{C}$ until analysis. The protein concentration was determined according to the method of Bradford (BioRad, Veenendaal, the Netherlands). 
NF- $\kappa$ B activity was measured using the TransAM NF- $\kappa B$ p50 Activation Assay (Active Motif, Rixensart, Belgium) and expressed as $\mu \mathrm{g}$ Jurkat NF- $\kappa \mathrm{B}$ equivalents per $\mu \mathrm{g}$ nuclear protein.

\section{Blood based cytokine production}

At $\mathrm{t}=0,43 \mathrm{~min}, 90 \mathrm{~min}$, and $24 \mathrm{~h}$ after exercise, the blood based TNF $\alpha$, IL-10 and IL-6 production was determined after stimulating whole blood for $24 \mathrm{~h}$ with $0.1 \mathrm{ng} / \mathrm{ml}$ lipopolysaccharide (LPS). TNF $\alpha$, IL-10 and IL-6 concentrations were quantified using Pelikine Compact human Enzyme linked immuno sorbent assay (ELISA) kits (CBL/Sanquin, The Netherlands).

\section{Urine collection}

Morning urine was collected on the test day and on the morning of the day after the test day. Aliquots of urine were stored at $-80^{\circ} \mathrm{C}$ until analysis of $\mathrm{F}_{2 \alpha^{-}}$ isoprostanes and creatinine.

\section{Urine analysis}

$\mathrm{F}_{2 \alpha}$-isoprostanes (8-iso $\mathrm{PGF}_{2 \alpha}$ ) in urine were analysed by an enzyme immunoassay (Cayman Chemical, Ann Arbor, MI, USA) and were related to creatinine values.

\section{Muscle biopsies}

Muscle biopsy samples were taken 30 min before the start of the exercise, i.e. prior to hydroxytyrosol or placebo ingestion, and $30 \mathrm{~min}$ after exercise, to determine the acute effects of exercise. Muscle biopsies were obtained from the middle region of the $\mathrm{m}$. vastus lateralis (15 $\mathrm{cm}$ above the patella) and approximately $3 \mathrm{~cm}$ below entry through the fascia using the percutaneous needle biopsy technique. The second muscle biopsy was taken from the contra-lateral leg. Muscle samples were freed from any visible nonmuscle material and rapidly frozen in liquid nitrogen. Muscle biopsies were stored at $-80^{\circ} \mathrm{C}$ until analysis.

\section{Preparation of muscle homogenates}

Frozen muscle biopsies were weighed and subsequently ground with a liquid nitrogen cooled mortar. The powder was resuspended in demineralised water to a final concentration of $250 \mathrm{mg}$ tissue/ml. SSA (final concentration of $1.3 \%$ ) was added to a small part of the homogenates, for the analysis of GSH and GSSG. The homogenates were directly frozen in liquid nitrogen and were 
subsequently stored at $-80^{\circ} \mathrm{C}$ until analysis. The homogenates were centrifuged at $14,000 \cdot \mathrm{g}$ at $4^{\circ} \mathrm{C}$ for $3 \mathrm{~min}$. The muscle biopsy supernatant was used for analysis.

\section{Muscle biopsy analyses}

Glutathione S-transferase (GST) activity, glutathione reductase activity, uric acid and protein levels in muscle biopsies were determined according to the methods of Julicher et al. (30). GST activity was determined by the enzymatic reaction of chlorodinitrobenzene with GSH. The increase in the formation of the coloured product of the reaction was measured at $340 \mathrm{~nm}$ for $2 \mathrm{~min}$. Glutathione reductase activity was measured by determining the consumption of nicotinamide adenine dinucleotide phosphate (NADPH) in the enzymatic reduction of GSSG to GSH. The decrease in absorption at $340 \mathrm{~nm}$ was measured during $2 \mathrm{~min}$. Skeletal muscle GSH and GSSG concentrations were measured according to the method for the determination of erythrocyte GSH and GSSG concentrations, as described above. Uric acid concentrations were determined in muscle biopsies of four subjects, because of the limited amount of muscle tissue of three subjects.

\section{Statistics}

All data are expressed as means \pm SEM. Analysis of variance (ANOVA) was applied to determine differences between hydroxytyrosol and placebo supplementation in plasma antioxidant concentrations and inflammatory markers. Sign tests were used to compare the effects of hydroxytyrosol and placebo supplementation on AUC and $t_{1 / 2}$ of lactate. Pre-versus post-exercise values were calculated for skeletal muscle antioxidants and urinary isoprostanes. The Wilcoxon signed-rank test was used to compare the effect of hydroxytyrosol with placebo. Statistical significance was set at $\mathrm{P}<0.10$.

\section{RESULTS}

\section{Plasma antioxidant capacity}

At $8.00 \mathrm{pm}$, the evening before the start of the exercise, $200 \mathrm{mg}$ hydroxytyrosol or placebo was given. The following morning, before the second intake of hydroxytyrosol or placebo, no differences between the hydroxytyrosol or placebo group in any of the parameters studied was observed, including the TEAC. Moreover, neither hydroxytyrosol nor hydroxytyrosol metabolites could be detected in plasma of the volunteers that had received hydroxytyrosol. 
After the second intake of placebo or hydroxytyrosol, plasma samples were taken at relatively short time intervals. It was found that $200 \mathrm{mg}$ hydroxytyrosol resulted in a considerable increase in plasma TEAC (Figure 2A) $(\mathrm{P}<0.05)$. Thirty minutes after hydroxytyrosol intake, the TEAC of plasma increased with $17 \%$. No significant changes in TEAC were observed after intake of the placebo (Figure 2A).
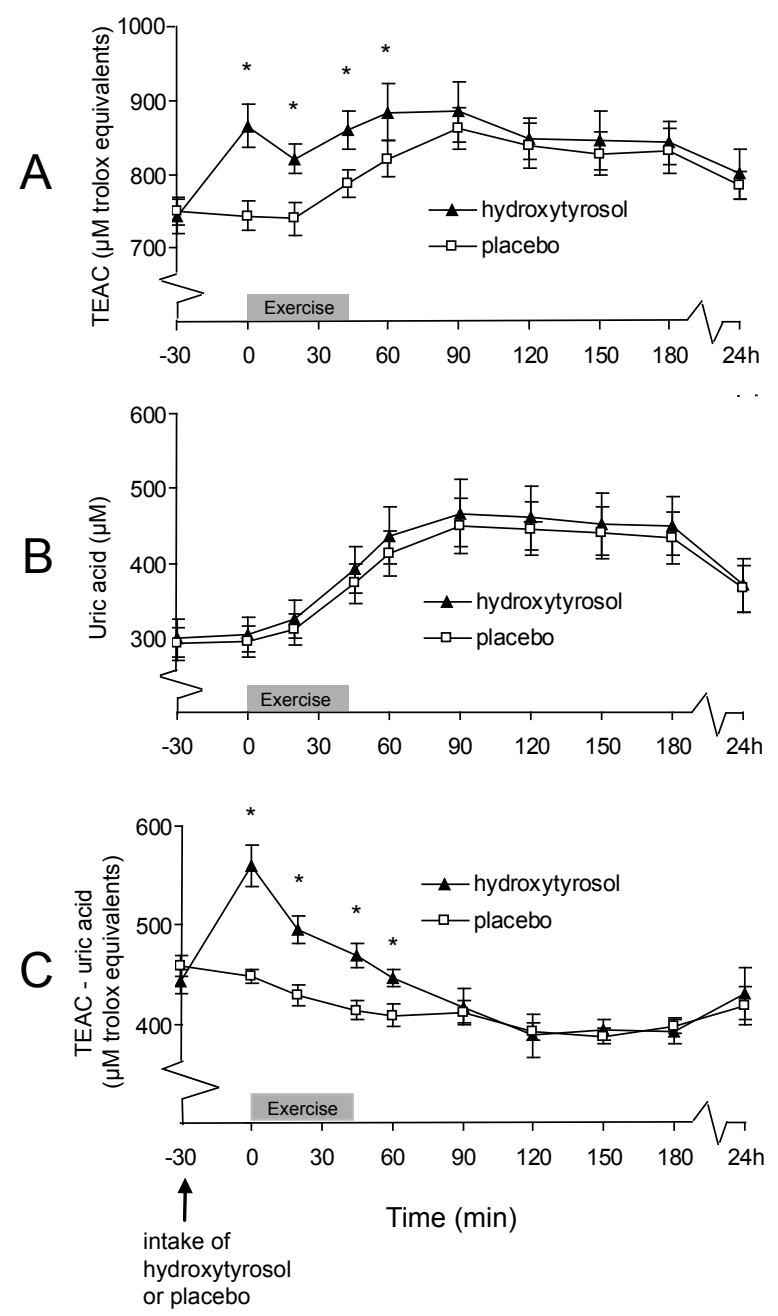

Figure 2. The effect of hydroxytyrosol on plasma TEAC values (panel A), uric acid concentrations (panel B) and TEAC values, corrected for uric acid (panel C) during resistance exercise (duration is indicated by the grey bar) and subsequent recovery. Data provided are means \pm SEM.

* Significantly different from placebo $(\mathrm{P}<0.05)$. 
Exercise also considerably increased the TEAC. At $\mathrm{t}=0$ (i.e. $30 \mathrm{~min}$ after intake of hydroxytyrosol), $\mathrm{t}=20 \mathrm{~min}$, and directly after exercise ( $\mathrm{t}=43 \mathrm{~min}$ and $\mathrm{t}=60$ min), hydroxytyrosol supplementation resulted in higher TEAC values, compared to placebo $(\mathrm{P}<0.05)$. During further recovery, TEAC values after hydroxytyrosol and placebo supplementation became similar (Figure 2A). The increase in uric acid concentrations during exercise was similar after hydroxytyrosol and placebo supplementation (Figure 2B). Fifty minutes after exercise, the uric acid concentration reached a maximum value after which it slightly decreased.
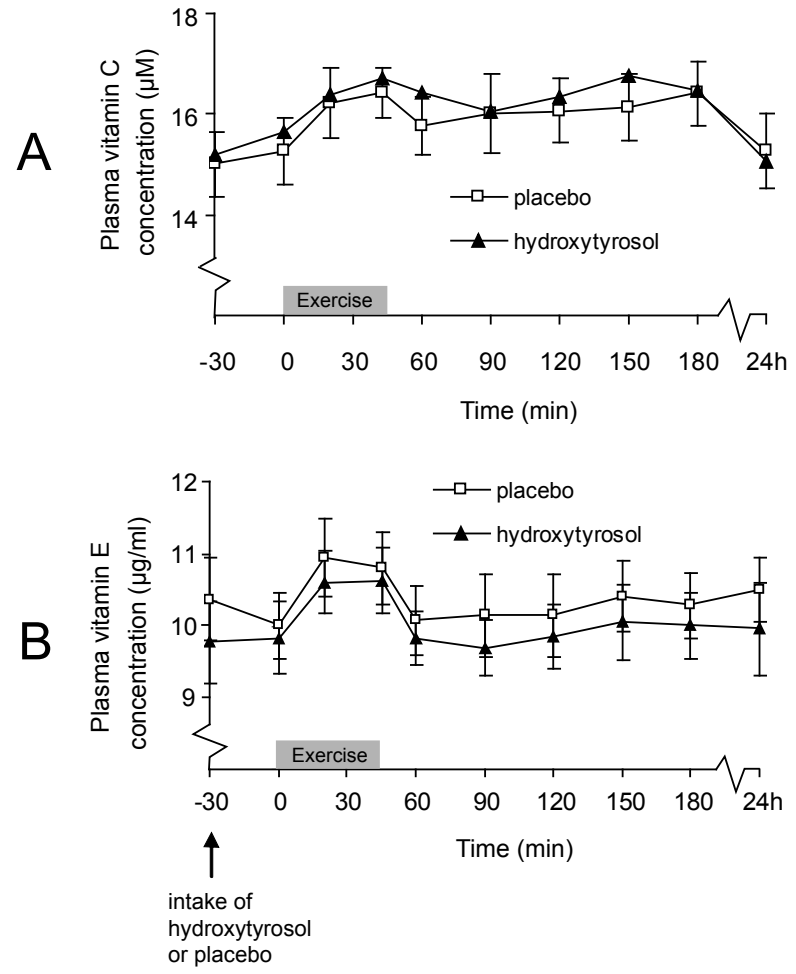

Figure 3. Plasma vitamin C (panel A) and vitamin E (panel B) concentrations during resistance exercise (duration is indicated by the grey bar) and subsequent recovery, after hydroxytyrosol and placebo supplementation. Data provided are means \pm SEM.

The antioxidant uric acid significantly contributes to the TEAC. This contribution of uric acid to the TEAC can be calculated. Subtracting the contribution of uric acid from the TEAC gives the 'uric acid-subtracted TEAC' value. The 'uric acid-subtracted TEAC' decreased during exercise. It was found that hydroxytyrosol supplementation resulted in an increased 'uric acid-subtracted TEAC', 
compared to placebo, at the start of the exercise $(t=0)$, during exercise $(t=20$ $\mathrm{min}$ ) and directly after exercise ( $\mathrm{t}=43 \mathrm{~min}$ and $60 \mathrm{~min})(\mathrm{P}<0.05$; Figure $2 \mathrm{C})$. During further recovery, 'uric acid-subtracted TEAC' values after hydroxytyrosol and placebo supplementation were similar (Figure 2C).

Hydroxytyrosol is known to be extensively metabolized e.g. by conjugation to glucuronic acid, sulphate or glutathione (31). Accordingly, the plasma levels of free hydroxytyrosol were below the lower limit of quantification $(0.2 \mu \mathrm{M}$; HPLC). Total conjugated hydroxytyrosol was determined in plasma samples subjected to acidic hydrolysis. Peak concentration of total conjugated hydroxytyrosol was approximately $5 \mu \mathrm{M}$. Hydroxytyrosol is also metabolized to homovanillic alcohol (31). The maximal plasma concentration of homovanillic alcohol was approximately $1 \mu \mathrm{M}$.

Plasma vitamin C and vitamin E concentrations temporarily elevated during exercise. Hydroxytyrosol supplementation didn't affect plasma vitamin E and vitamin $\mathrm{C}$ levels during exercise and recovery (Figure $3 \mathrm{~A}$ and B).

Erythrocyte GSH levels also temporarily increased during exercise. Hydroxytyrosol didn't affect the erythrocyte GSH levels during exercise and recovery. The erythrocyte GSSG concentration remained during exercise at baseline value, after both hydroxytyrosol and placebo supplementation.

\section{Antioxidant activity in skeletal muscle}

The increase in GSH concentrations in skeletal muscle, 30 min after exercise, was $5.5 \pm 4.3 \mathrm{nmol} / \mathrm{mg}$ protein. The supplementation of hydroxytyrosol increased skeletal muscle GSH levels after exercise. After hydroxytyrosol supplementation, the increase in GSH levels was $14.4 \pm 7.6 \mathrm{nmol} / \mathrm{mg}$ protein (Figure $4 \mathrm{~A})(\mathrm{P}<0.10)$. Neither exercise, nor hydroxytyrosol did affect GSSG concentrations in skeletal muscle (Figure 4C).

Glutathione reductase activity in skeletal muscle increased after exercise on average with $4.9 \pm 2.2 \mathrm{mU} / \mathrm{mg}$ protein (Figure $4 \mathrm{~B}$ ). The increase in glutathione reductase activity induced by exercise was higher after hydroxytyrosol supplementation, i.e. an increment of $7.8 \pm 2.9 \mathrm{mU} / \mathrm{mg}$ protein was found (Figure $4 \mathrm{~B}$ ) $(\mathrm{P}<0.10)$. The increase in skeletal muscle GST activity by exercise was similar after hydroxytyrosol and placebo supplementation (Figure 4D). The increase in uric acid concentrations in skeletal muscle after exercise was also comparable after placebo or hydroxytyrosol supplementation (Figure 4E).

\section{8-iso PGF $_{2 a}$ levels in urine}

The increase in the concentration of 8-iso $\mathrm{PGF}_{2 \alpha}$ in urine during exercise was similar after hydroxytyrosol and placebo supplementation. In case of placebo, urinary 8-iso $\mathrm{PGF}_{2 \alpha}$ increased from $0.117 \pm 0.021 \mathrm{nmol} / \mathrm{mmol}$ creatinine pre- 
exercise to $0.164 \pm 0.030 \mathrm{nmol} / \mathrm{mmol}$ creatinine post-exercise (Figure 5). After hydroxytyrosol supplementation, urinary 8-iso $\mathrm{PGF}_{2 \alpha}$ increased from $0.107 \pm$ $0.014 \mathrm{nmol} / \mathrm{mmol}$ creatinine pre-exercise to $0.165 \pm 0.028 \mathrm{nmol} / \mathrm{mmol}$ creatinine post-exercise (Figure 4F).
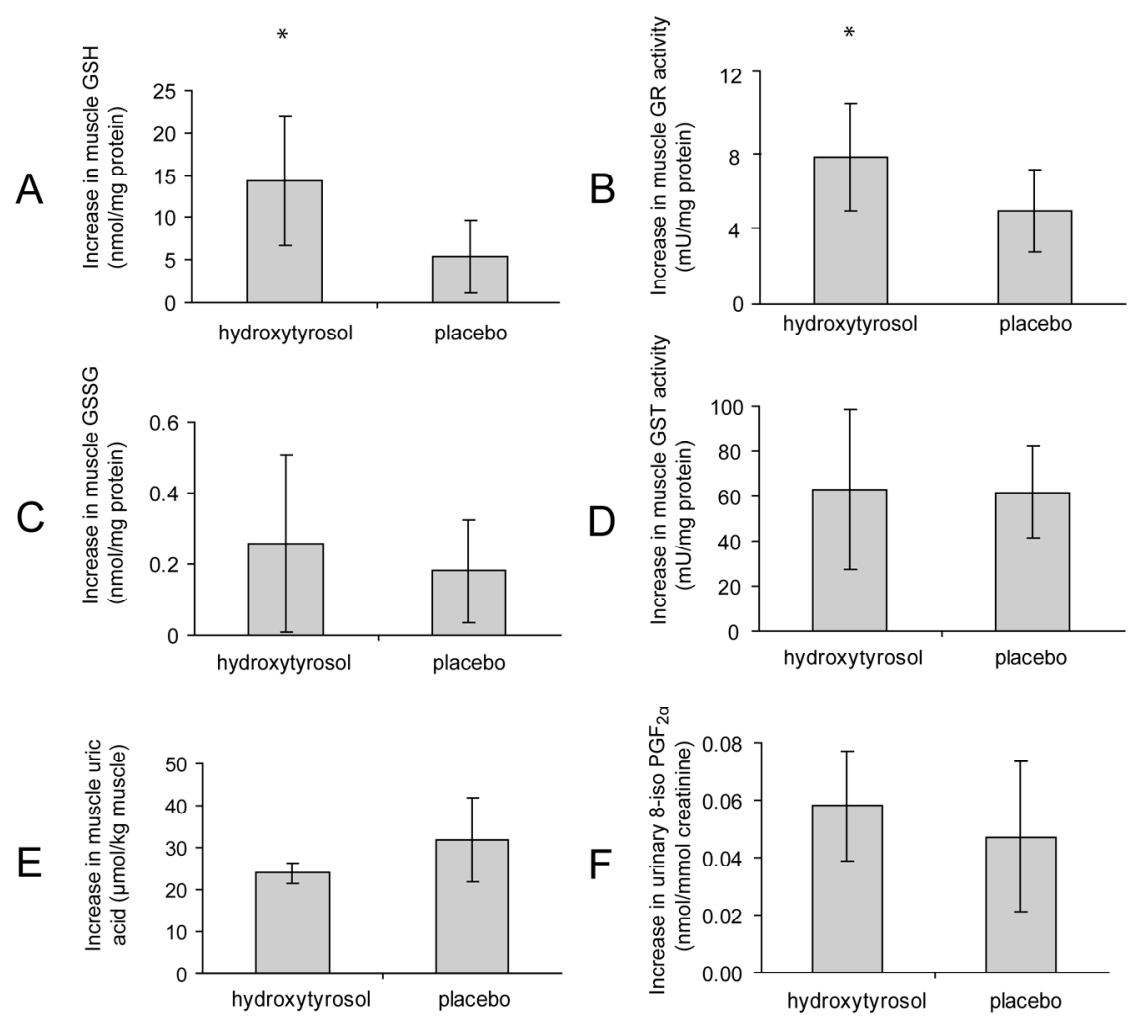

Figure 4. The effect of hydroxytyrosol on exercise-induced increases in skeletal muscle GSH (panel A), glutathione reductase (GR) activity (panel B), GSSG (panel C), GST activity (panel D), uric acid (panel E) and urinary isoprostanes (panel F). Data provided are means \pm SEM.

* Significantly different from placebo $(\mathrm{P}<0.10)$.

\section{Inflammatory markers}

Exercise did not induce changes in NF-kB activity in PBMCs. The supplementation of hydroxytyrosol also didn't affect the NF-kB activity in PBMCs. Furthermore, the ex vivo LPS-induced production of TNF $\alpha$, IL-10 and IL- 6 was neither affected by exercise, nor by hydroxytyrosol supplementation (data not shown). 


\section{Lactate}

Hydroxytyrosol supplementation tended to decrease the plasma peak concentration of lactate directly after exercise ( $\mathrm{t}=43 \mathrm{~min})(10.2 \pm 1.2$ vs $11.0 \pm 1.0$ $\mathrm{mM}$; Figure 5A). The AUC, reflecting the production of lactate, and the $t_{1 / 2}$ of lactate were calculated for each volunteer. This reveals that there are large inter-individual differences in both $A \cup C$ and $t_{1 / 2}$. The AUC values ranged from 296 to 783 and the $t_{1 / 2}$ of lactate ranged from 12 to $27 \mathrm{~min}$. Hydroxytyrosol decreased the AUC of lactate from $560 \pm 65$ to $522 \pm 67$ (Figure 5B) $(P=0.063)$.

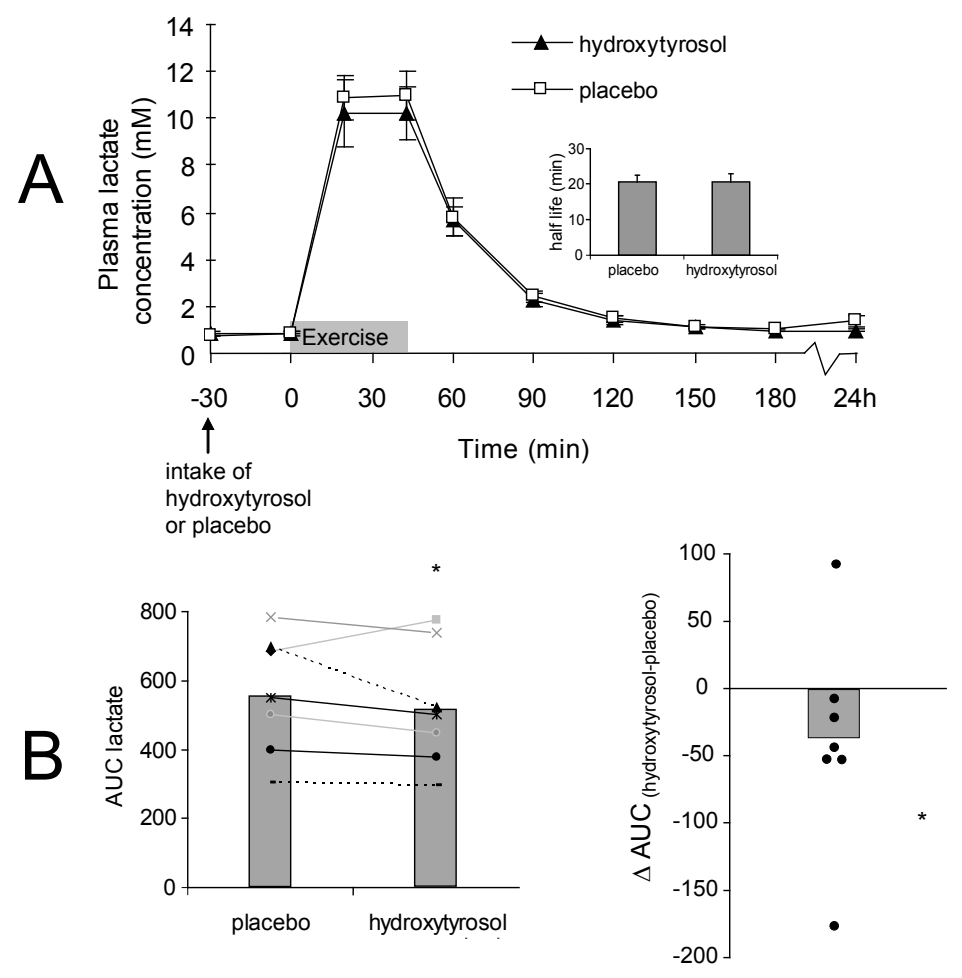

Figure 5. The effect of hydroxytyrosol on lactate levels during resistance exercise (duration is indicated by the grey bar) and subsequent recovery (panel A). The $t_{1 / 2}$ of lactate after hydroxytyrosol or placebo supplementation is shown in the insert of panel A. The effect of hydroxytyrosol on the AUC of lactate is shown in panel B. Data provided are means \pm SEM (panel A) or individual values plus mean values (grey bars) (panel B).

$\triangle \mathrm{AUC}=\mathrm{A} \cup \mathrm{C}_{\text {hydroxytyrosol }}-\mathrm{AUC}_{\text {placebo }}$

* Significantly different from placebo $(\mathrm{P}<0.10)$. 
Hydroxytyrosol did not affect the clearance, i.e. the elimination of lactate out of the blood compartment as the $t_{1 / 2}$ of lactate was identical after hydroxytyrosol and placebo supplementation $\left(\mathrm{t}_{1 / 2}=20.6 \pm 2.2\right.$ and $20.5 \pm 2.0$ min respectively; insert Figure 5A). The average $t_{1 / 2}$ of lactate of $21 \mathrm{~min}$ corresponds to values previously reported (32).

\section{DISCUSSION}

The interest in antioxidant supplements in the field of exercise has largely increased. The rationale to use antioxidants during exercise is to empower the endogenous antioxidant defence in order to cope with the increased radical flux induced by exercise. This may prevent muscle damage, which is expected to enhance performance and shorten the recovery period. Up to date, the effect of antioxidant supplementation during exercise is inconclusive.

Muscle endogenous antioxidant defence systems are up-regulated in response to exercise $(13,33)$. ROS produced during exercise may act as signals to increase the production of antioxidant enzymes. NF- $\mathrm{kB}$ and mitogen-activated protein kinase (MAPK) are two major oxidative stress-sensitive signal transduction pathways that have been shown to activate the gene expression of a number of antioxidant enzymes $(34,35)$. In literature, it has been speculated that antioxidant supplementation might block this adaptive response to exercise (33).

In the present study the effect of the olive antioxidant hydroxytyrosol during exercise was studied. Hydroxytyrosol was chosen because of its excellent antioxidant characteristics. It is a potent scavenger of reactive oxygen and nitrogen species (17). Furthermore, hydroxytyrosol has been shown to provide protection against oxidative damage to all kinds of cells in vitro $(18,20-22)$. Hydroxytyrosol also displays antioxidant effects in vivo. For example, it provides protection against the oxidation of low density lipoprotein $(\mathrm{LDL})$, one of the key steps in the initiation of atherosclerosis (36-39).

The effect of hydroxytyrosol on skeletal muscle has not been studied in detail so far. Most of the research on hydroxytyrosol is focused on its beneficial cardiovascular properties. Recently, we have shown that hydroxytyrosol efficiently protects against oxidative stress-induced reduction in force production of rat skeletal muscle in vitro (chapter 5 of the thesis). The aim of the present study was to investigate the in vivo effects of hydroxytyrosol in untrained, healthy volunteers during a single session of strenuous resistance type exercise.

The present study shows that the supplementation of hydroxytyrosol boosts the antioxidant defence system, as can be deduced from the large increase in TEAC of plasma. The TEAC increased by more than $100 \mu \mathrm{M}$ trolox equivalents, while 
only approximately $5 \mu \mathrm{M}$ of hydroxytyrosol metabolites could be detected in plasma, which contribute for maximally $10 \mu \mathrm{M}$ trolox equivalents to the TEAC. Apparently, the antioxidant activity of hydroxytyrosol itself and its known metabolites can explain only a small part of the increase in TEAC after hydroxytyrosol supplementation. The molecular mechanism responsible for the high increase in TEAC remains to be elucidated. It is possible that hydroxytyrosol is converted to other, unknown metabolites with high antioxidant activity. It should be noted that antioxidants do not act in isolation; they synergize to form an intricate network of antioxidants. It is possible that hydroxytyrosol regenerates other antioxidants that are present in the antioxidant network.

Importantly, hydroxytyrosol did not hamper the exercise-induced adaptive antioxidant response. The exercise-induced increase in erythrocyte GSH and plasma vitamin E, vitamin C, and uric acid concentrations was not affected by hydroxytyrosol supplementation. Moreover, the glutathione defence system in skeletal muscle was up-regulated by hydroxytyrosol, as shown by an elevation in GSH levels after exercise. Furthermore, the exercise induced increase in glutathione reductase activity was even higher after hydroxytyrosol supplementation, compared to placebo. This indicates that hydroxytyrosol rather boosts than blocks the exercise induced adaptive response.

Hydroxytyrosol supplementation didn't attenuate the rise in the concentration of 8-iso $\mathrm{PGF}_{2 \alpha}$ in urine $24 \mathrm{~h}$ after a single session of resistance exercise. Based on this result, hydroxytyrosol doesn't seem to be effective in protecting against ROS generated by exercise.

Several studies on the use of antioxidants to attenuate exercise-induced muscle injury and oxidative stress during exercise have been performed. Most of the research in this area is focused on common dietary antioxidants such as vitamin $\mathrm{E}$ and vitamin $\mathrm{C}$. With regard to endurance exercise, the effect of antioxidant supplements is not straightforward, as studies in this area show inconsistent results (16). Regarding a bout of resistance exercise, some reports suggest a potential beneficial role of antioxidant supplementation, whereas others indicate no benefit (15).

In general, the efficacy of an antioxidant to protect against exercise-induced oxidative stress depends on several methodological aspects, such as (I) the specific population studied, (II) the type of exercise, (III) the type of antioxidant, dosage, and timing of administration of the antioxidant, (IV) the biomarkers used, and ( $\mathrm{V}$ ) the sampling time.

Most studies reporting a benefit of antioxidant supplementation in attenuating muscle injury and oxidative stress following resistance exercise have included sedentary, non-resistance trained subjects. In trained subjects, endogenous antioxidant defences may be up-regulated, and, therefore, these individuals may not benefit greatly from exogenous antioxidant intake in order to reduce muscle injury (34). We selected untrained subjects for the present study, based 
on our speculation that oxidative damage would be greater in a non-trained group, thereby increasing the window of opportunity to detect an effect of hydroxytyrosol supplementation on oxidative damage and antioxidant capacity. Studies on the use of antioxidants during exercise have used several types of exercise, ranging from long-term endurance exercise to short-term resistance exercise. Aerobic endurance exercise will induce a different flux of radicals than anaerobic resistance exercise does (35). In designing the study, we speculated that the effect of an acute and high flux of radicals would be more sensitive to antioxidant supplementation than that of a more prolonged and moderate flux of radicals. In order to maximize the sensitivity of the study, a single session of strenuous resistance exercise was applied. It has been suggested that the effect of antioxidants depends on the degree of muscle damage (33). During moderate exercise, antioxidants might hamper the beneficial adaptive response. When strenuous exercise is applied, such as in the present study, antioxidants are expected to prevent oxidative stress-induced muscle injury (33).

The efficacy of the antioxidant studied also depends on the type of antioxidant, dose, and timing of the administration of the antioxidant. In the present study, hydroxytyrosol increased the TEAC of plasma with more than $100 \mu \mathrm{M}$ trolox equivalents, clearly demonstrating the high antioxidant potency of hydroxytyrosol in a dose of just $200 \mathrm{mg}$. For comparison, administration of $2 \mathrm{~g}$ of the flavonoid quercetin increased the TEAC merely by $15 \mu \mathrm{M}$ trolox equivalents (unpublished results). The increase in TEAC of plasma lasted only approximately 90 minutes after intake of hydroxytyrosol. Supplementing hydroxytyrosol not only 30 minutes before, but also directly after exercise would have prolonged the increase in TEAC, which might have been more effective, in retrospect.

Furthermore, the observed efficacy of an antioxidant will also depend on the biomarkers used. Plasma protein carbonyl content is often used as a biomarker for exercise induced oxidative damage $(6,7,9)$. We also intended to include this biomarker. However, in our hands, the routinely applied procedure for determination of protein carbonyl content lacked sufficient specificity, due to interference by pyruvate (data not shown).

The observed effect of an antioxidant is also dependent on sampling time. In the present study the sampling time was limited to $24 \mathrm{~h}$ post exercise. Up to $24 \mathrm{~h}$ post exercise, inflammatory markers were neither affected by exercise, nor by hydroxytyrosol supplementation. It is possible that in a more long term study design, effects of exercise or hydroxytyrosol would have been detected. The anti-inflammatory effect of hydroxytyrosol has been shown both in preclinical and clinical studies (40-42).

It was expected that the large increase in TEAC of plasma and the upregulation of the glutathione defence system in muscle due to hydroxytyrosol would have 
reduced oxidative stress. In spite of the inability to attenuate the exerciseinduced rise in urinary isoprostanes, hydroxytyrosol did reduce the production of lactate during exercise. Sastre et al. have reported a positive correlation between oxidative stress, namely the GSSG to GSH ratio, and the lactate to pyruvate ratio during exhaustive physical exercise (43). Recently, it has been shown that long-term co-supplementation (90 days) with vitamin $E, \beta$-carotene and vitamin $C$ reduced the maximal blood lactate concentration during exercise. The present study shows that also short-term supplementation with hydroxytyrosol is able to reduce the production of lactate during strenuous exercise. Although the mechanism is still unclear, it is obvious that antioxidants are able to affect lactate levels during exercise. Additional research is needed to clarify this finding.

The present study does not support the alleged antagonistic action of antioxidants on muscle cell adaptation. On the contrary, hydroxytyrosol rather strengthens than blocks the endogenous antioxidant defence during a single session of strenuous resistance type exercise in untrained men. In spite of the inability of hydroxytyrosol to attenuate the exercise-induced rise in urinary isoprostanes, hydroxytyrosol considerably increases the TEAC of plasma and attenuates the rise in plasma lactate during exercise.

\section{Acknowledgements}

We would like to thank DSM Food Specialties, Nico Crombach, Marie-José Drittij, Marc Fischer, René Koopman, Ralph Manders and Bart Pennings for their valuable assistance. 


\section{REFERENCES}

1. Finaud J, Lac G, Filaire E. Oxidative stress : relationship with exercise and training. Sports Med. 2006;36(4):327-58.

2. Jackson MJ, Pye D, Palomero J. The production of reactive oxygen and nitrogen species by skeletal muscle. J Appl Physiol. 2007;102(4):1664-1670.

3. Reid MB. Invited Review: redox modulation of skeletal muscle contraction: what we know and what we don't. J Appl Physiol. 2001;90(2):724-31.

4. Sjodin B, Hellsten Westing Y, Apple FS. Biochemical mechanisms for oxygen free radical formation during exercise. Sports Med. 1990;10(4):236-54.

5. Akova B, Surmen-Gur E, Gur H, Dirican M, Sarandol E, Kucukoglu S. Exercise-induced oxidative stress and muscle performance in healthy women: role of vitamin $E$ supplementation and endogenous oestradiol. Eur J Appl Physiol. 2001;84(1-2):141-7.

6. Bloomer RJ, Falvo MJ, Fry AC, Schilling BK, Smith WA, Moore CA. Oxidative stress response in trained men following repeated squats or sprints. Med Sci Sports Exerc. 2006;38(8):1436-42.

7. Bloomer RJ, Fry AC, Falvo MJ, Moore CA. Protein carbonyls are acutely elevated following single set anaerobic exercise in resistance trained men. J Sci Med Sport. 2007;10:411-417.

8. Bloomer RJ, Goldfarb AH. Anaerobic exercise and oxidative stress: a review. Can J Appl Physiol. 2004;29(3):245-63.

9. Bloomer RJ, Goldfarb AH, Wideman L, McKenzie MJ, Consitt LA. Effects of acute aerobic and anaerobic exercise on blood markers of oxidative stress. J Strength Cond Res. 2005;19(2):27685.

10. Bryer SC, Goldfarb AH. Effect of high dose vitamin C supplementation on muscle soreness, damage, function, and oxidative stress to eccentric exercise. Int J Sport Nutr Exerc Metab. 2006;16(3):270-80.

11. Goldfarb AH, Bloomer RJ, McKenzie MJ. Combined antioxidant treatment effects on blood oxidative stress after eccentric exercise. Med Sci Sports Exerc. 2005;37(2):234-9.

12. Ramel A, Wagner $\mathrm{KH}$, Elmadfa I. Plasma antioxidants and lipid oxidation after submaximal resistance exercise in men. Eur J Nutr. 2004;43(1):2-6.

13. Rietjens SJ, Beelen M, Koopman R, van Loon LJ, Bast A, Haenen GR. A single session of resistance exercise induces oxidative damage in untrained men. Med Sci Sports Exerc. 2007;39(12):2145-51.

14. Vollaard NB, Shearman JP, Cooper CE. Exercise-induced oxidative stress:myths, realities and physiological relevance. Sports Med. 2005;35(12):1045-62.

15. Bloomer RJ. The role of nutritional supplements in the prevention and treatment of resistance exercise-induced skeletal muscle injury. Sports Med. 2007;37(6):519-32.

16. Williams SL, Strobel NA, Lexis LA, Coombes JS. Antioxidant requirements of endurance athletes: implications for health. Nutr Rev. 2006;64(3):93-108.

17. Rietjens SJ, Bast A, Haenen GRMM. New insights into controversies on the antioxidant potential of the olive oil antioxidant hydroxytyrosol. J Agric Food Chem. 2007;55(18):7609-14.

18. Rietjens SJ, Bast A, de Vente J, Haenen GR. The olive oil antioxidant hydroxytyrosol efficiently protects against the oxidative stress induced impairment of the $\mathrm{NO}$ ' response of isolated rat aorta. Am J Physiol Heart Circ Physiol. 2007;292:H1931-6.

19. Visioli F, Bellomo G, Montedoro G, Galli C. Low density lipoprotein oxidation is inhibited in vitro by olive oil constituents. Atherosclerosis. 1995;117(1):25-32.

20. Goya L, Mateos R, Bravo L. Effect of the olive oil phenol hydroxytyrosol on human hepatoma HepG2 cells : Protection against oxidative stress induced by tert-butylhydroperoxide. Eur J Nutr. 2007.

21. Manna C, Galletti P, Cucciolla V, Moltedo O, Leone A, Zappia V. The protective effect of the olive oil polyphenol (3,4-dihydroxyphenyl)-ethanol counteracts reactive oxygen metaboliteinduced cytotoxicity in Caco-2 cells. J Nutr. 1997;127(2):286-92. 
22. Manna C, Galletti P, Cucciolla V, Montedoro G, Zappia V. Olive oil hydroxytyrosol protects human erythrocytes against oxidative damages. J Nutr Biochem. 1999;10:159-165.

23. Visioli F, Galli C, Plasmati E, et al. Olive phenol hydroxytyrosol prevents passive smokinginduced oxidative stress. Circulation. 2000;102(18):2169-71.

24. Visioli F, Caruso D, Galli C, Viappiani S, Galli G, Sala A. Olive oils rich in natural catecholic phenols decrease isoprostane excretion in humans. Biochem Biophys Res Commun. 2000;278(3):797-9.

25. Koopman R, Manders RJ, Zorenc AH, et al. A single session of resistance exercise enhances insulin sensitivity for at least $24 \mathrm{~h}$ in healthy men. Eur J Appl Physiol. 2005;94(1-2):180-7.

26. Koopman R, Manders RJ, Jonkers RA, Hul GB, Kuipers H, van Loon LJ. Intramyocellular lipid and glycogen content are reduced following resistance exercise in untrained healthy males. Eur J Appl Physiol. 2006;96(5):525-34.

27. Gutmann I, Wahlefeld A. L-(+)-Lactate, determination with lactate dehydrogenase and NAD. In: Bergmeyer H, ed. Methods in enzymatic analysis. 2 ed. New York: Academic; 1974:14641468.

28. Rowland M, Tozer TN. Clinical Pharmacokinetics, Concepts and Applications Lippincott Williams and Wilkins; 1995.

29. Fischer MA, Gransier TJ, Beckers LM, Bekers O, Bast A, Haenen GR. Determination of the antioxidant capacity in blood. Clin Chem Lab Med. 2005;43(7):735-40.

30. Julicher RH, Sterrenberg L, Haenen GR, Bast A, Noordhoek J. Sex differences in the cellular defence system against free radicals from oxygen or drug metabolites in rat. Arch Toxicol. 1984;56(2):83-6.

31. Miro Casas E, Covas MI, Farre M, et al. Hydroxytyrosol disposition in humans. Clin Chem. 2003;49(6 Pt 1):945-52.

32. Dotan R, Ohana S, Bediz C, Falk B. Blood lactate disappearance dynamics in boys and men following exercise of similar and dissimilar peak-lactate concentrations. J Pediatr Endocrinol Metab. 2003;16(3):419-29.

33. Gomez-Cabrera M, Domenech E, Vina J. Moderate exercise is an antioxidant: Upregulation of antioxidant genes by training. Free Radic Biol Med. 2008;44(2):126-31.

34. Ji LL. Modulation of skeletal muscle antioxidant defense by exercise: Role of redox signaling. Free Radic Biol Med. 2008;44(2):142-52.

35. Pattwell DM, Jackson MJ. Contraction-induced oxidants as mediators of adaptation and damage in skeletal muscle. Exerc Sport Sci Rev. 2004;32(1):14-8.

36. Covas MI, Nyyssonen K, Poulsen HE, et al. The effect of polyphenols in olive oil on heart disease risk factors: a randomized trial. Ann Intern Med. 2006;145(5):333-41.

37. Fito $M$, Cladellas $M$, de la Torre $R$, et al. Antioxidant effect of virgin olive oil in patients with stable coronary heart disease: a randomized, crossover, controlled, clinical trial. Atherosclerosis. 2005;181(1):149-58.

38. Marrugat J, Covas MI, Fito M, et al. Effects of differing phenolic content in dietary olive oils on lipids and LDL oxidation--a randomized controlled trial. Eur J Nutr. 2004;43(3):140-7.

39. Weinbrenner $\mathrm{T}$, Fito $\mathrm{M}$, de la Torre $\mathrm{R}$, et al. Olive oils high in phenolic compounds modulate oxidative/antioxidative status in men. J Nutr. 2004;134(9):2314-21.

40. Bitler CM, Matt K, Irving M, et al. Olive extract supplement decreases pain and improves daily activities in adults with osteoarthritis and decreases plasma homocysteine in those with rheumatoid arthritis. Nutr Research. 2007;27:470-477.

41. Bitler CM, Viale TM, Damaj B, Crea R. Hydrolyzed olive vegetation water in mice has antiinflammatory activity. J Nutr. 2005;135(6):1475-9.

42. Martinez-Dominguez E, de la Puerta R, Ruiz-Gutierrez V. Protective effects upon experimental inflammation models of a polyphenol-supplemented virgin olive oil diet. Inflamm Res. 2001;50(2):102-6.

43. Sastre J, Asensi M, Gasco E, et al. Exhaustive physical exercise causes oxidation of glutathione status in blood: prevention by antioxidant administration. Am J Physiol. 1992;263(5 Pt 2):R9925. 


\section{CHAPTER 7}

\section{General discussion}

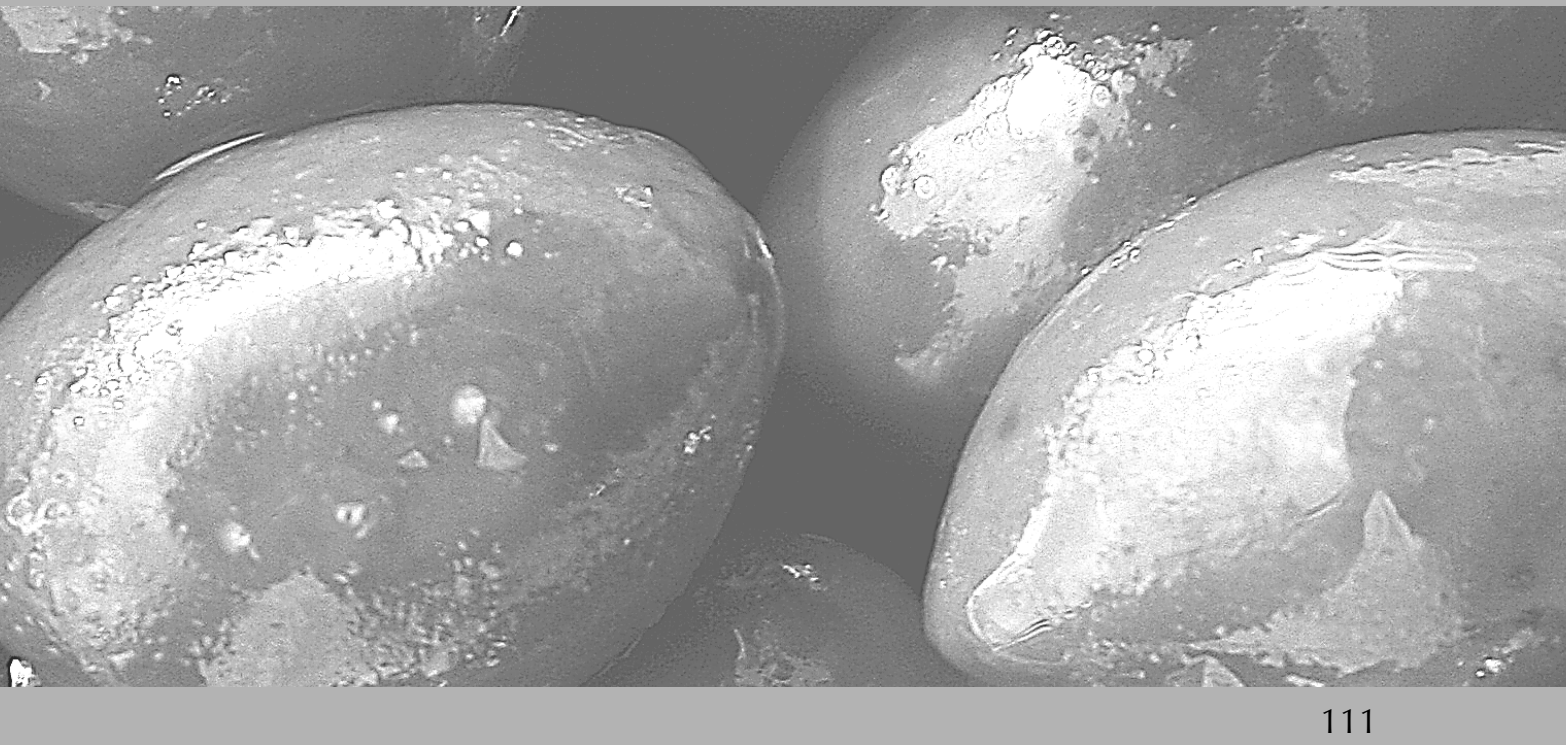




\section{GENERAL DISCUSSION}

Over the past decades, the interest in antioxidants has largely increased. Antioxidants are considered to support health. Consequently, the market for supplements containing antioxidants with health promoting claims is growing. The general purpose of the intake of these 'exogenous' antioxidants is to empower the endogenous antioxidant system to combat ROS-mediated damage.

Phenolic antioxidants present in olive oil have gained a lot of interest. Current evidence indicates that olive oil phenolic compounds provide beneficial health effects, e.g. on the cardiovascular system. Hydroxytyrosol is one of the main phenolic compounds present in virgin olive oil and is endowed with a wide range of antioxidant effects.

During the production of olive oil, large quantities of olive oil mill wastewater (OMWW) are produced. This wastewater constitutes a serious environmental problem, as it has severe negative impact on soil and water quality. OMWW has a higher content of phenolic compounds than olive oil itself. The recovery of phenolic compounds from OMWW is very valuable, not only from an environmental point of view, but also because these compounds might be useful in the pharmaceutical, food and cosmetic industry. Supplements with health promoting claims are currently being developed from OMWW. The content of phenolic compounds present in some of these extracts is 300-500 times higher than that in olive oil itself.

This thesis focuses on the health benefits of hydroxytyrosol from its mechanism of action to its use as nutraceutical. The present thesis touches upon two specific aspects of hydroxytyrosol. Firstly, the health benefit of hydroxytyrosol on the cardiovascular system was further explored (chapter 2 and 3). Secondly, the protective effect of hydroxytyrosol against exercise induced oxidative stress in skeletal muscle was studied (chapter 4 - 6).

\section{Effect of hydroxytyrosol on the cardiovascular system}

The molecular mechanism of the beneficial cardiovascular effects of hydroxytyrosol has not been fully elucidated yet. In chapter 2 , the antioxidant profile of hydroxytyrosol was studied in detail. The intrinsic antioxidant activity forms the fundament for the physiological impact of hydroxytyrosol. Regarding the antioxidant profile of hydroxytyrosol some controversies exist. For example, the reports on superoxide radical $\left(\mathrm{O}_{2}{ }^{\circ}\right)$ scavenging are ambiguous. In chapter 2 it was shown that hydroxytyrosol is a potent scavenger of hydroxyl radicals $\left(\mathrm{OH}^{\circ}\right)$, peroxynitrite $(\mathrm{ONOOH})$, and $\mathrm{O}_{2}{ }^{\circ}$, but not of hypochlorous acid $(\mathrm{HOCl})$ and hydrogen peroxide $\left(\mathrm{H}_{2} \mathrm{O}_{2}\right)$. 
Also regarding the protective effect of hydroxytyrosol against low density lipoprotein $(\mathrm{LDL})$ oxidation some controversies exist. The oxidation of $\mathrm{LDL}$ is a key factor in the initiation of atherosclerosis. Hydroxytyrosol efficiently protects against LDL oxidation in vitro and in vivo. However, no protective effect of hydroxytyrosol is usually demonstrated ex vivo against the oxidation of LDL isolated from humans after hydroxytyrosol consumption.

Chapter 2 shows that this controversy is due to the isolation of LDL, which greatly reduces the protective effect of hydroxytyrosol against LDL oxidation. It was established that hydroxytyrosol is an amphiphilic compound, present in the aqueous as well as the lipophilic environment in comparable concentrations. During the LDL isolation procedure, most of the hydroxytyrosol is lost due to its amphiphilic nature. This indicates that the reduction in the protective effect of hydroxytyrosol might be due to the loss of hydroxytyrosol from LDL during the LDL isolation procedure. Therefore, in ex vivo experiments the effect of hydroxytyrosol is generally underestimated because of the LDL isolation procedure. Several studies have been published that are consistent in showing a protective effect of hydroxytyrosol against LDL oxidation in vivo.

In addition to oxidative modifications of LDL, oxidative damage to endothelial cells may also affect cardiovascular health. Under physiological conditions, endothelial $\mathrm{NO}^{*}$ is of pivotal importance in the regulation of the vascular tone. Oxidative damage to the delicate $\mathrm{NO}^{\bullet}$ system in endothelial cells might disturb the vascular tone. A diminished $\mathrm{NO}^{\bullet}$ mediated vasorelaxation leads to a rise in blood pressure, which is a well-established risk factor for the development or progression of atherosclerosis.

In chapter 3, the effect of oxidative stress on the $\mathrm{NO}^{\circ}$-mediated vasorelaxation of rat aorta was studied. Cumene hydroperoxide (CHP) was used to mimic oxidative stress induced by lipid hydroperoxides, which involves the formation of reactive species such as $\mathrm{OH}^{\circ}$. $\mathrm{CHP}$ impaired the $\mathrm{NO}^{*}$ mediated relaxation of rat aorta by the acetylcholine receptor agonist carbachol. This was due to a reduction in $\mathrm{NO}^{*}$ production. Chapter 3 shows that hydroxytyrosol efficiently protected the aorta against the $\mathrm{CHP}$ induced impairment of the $\mathrm{NO}^{\bullet}$ mediated relaxation. The protective effect of hydroxytyrosol might be explained by its potent scavenging activity, which can be attributed to its catechol moiety. The results in chapter 2 and 3 provide a molecular basis for the contribution of hydroxytyrosol to the cardiovascular health benefits of olive oil consumption.

\section{Effect of hydroxytyrosol on skeletal muscle}

The second focus of the thesis is on the potential of hydroxytyrosol to protect against oxidative stress in skeletal muscle. During exercise, the production of reactive oxygen and nitrogen species is significantly enhanced. Unbalanced production of reactive species will lead to oxidative damage to biomolecules, 
such as proteins and lipids. Fortunately, the body has an elaborate defence system of antioxidants that provides protection against these reactive species.

In chapter 4, it was investigated whether the endogenous antioxidant defence system is able to cope with the increased radical flux induced by exercise. It was expected that antioxidant levels would be reduced by increased consumption of antioxidants. Surprisingly, a single session of resistance type exercise was found to increase the concentration of several antioxidants in untrained healthy males. Plasma total antioxidant capacity (TEAC) significantly increased after exercise, which appeared to be predominantly due to elevated uric acid concentrations. Plasma vitamin C and vitamin E and erythrocyte glutathione (GSH) levels were also elevated during exercise. Also in skeletal muscle, uric acid levels were found to increase after exercise. Moreover, the activity of the antioxidant enzymes glutathione S-transferase (GST) and glutathione reductase was found to be significantly elevated by exercise.

ROS produced during exercise may act as signals to increase the production of antioxidant enzymes. Nuclear Factor kappa B (NF-kB) and mitogen-activated protein kinase (MAPK) are two major oxidative stress-sensitive signal transduction pathways that have been shown to activate the gene expression of a number of antioxidant enzymes. In spite of the exercise-induced adaptive increase in antioxidant capacity of blood and skeletal muscle, a single session of resistance exercise induced oxidative damage to lipids, as shown by an

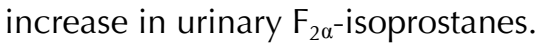

The fact that the endogenous antioxidant defence is not able to sufficiently cope with the increased radical flux induced by exercise provides a rationale for the use of antioxidant supplements during exercise. However, in literature, it has been speculated that antioxidant supplementation might block the adaptive antioxidant response to exercise. In chapter 5 and 6, it was investigated whether hydroxytyrosol would be a suitable candidate to protect against ROS generated during exercise.

Firstly, the antioxidant potential of hydroxytyrosol was investigated in isolated rat muscle strips (chapter 5). $\mathrm{H}_{2} \mathrm{O}_{2}$ was used to induce oxidative stress in rat skeletal muscle. The in vitro rat diaphragm preparation was applied, which is a well-established model to study skeletal muscle function in vitro. It was observed that hydroxytyrosol efficiently protects against the oxidative stressinduced impairment of force production during both short-term and fatiguing long-term electrical muscle stimulation.

In chapter 6, the in vivo protective effect of hydroxytyrosol against oxidative stress induced by a single session of resistance exercise was investigated in a double-blinded intervention study in untrained healthy volunteers. Supplementation with $200 \mathrm{mg}$ hydroxytyrosol resulted in a considerable increase in plasma total antioxidant capacity (TEAC). Hydroxytyrosol did not hamper the exercise-induced adaptive antioxidant response. The exercise-induced increase 
in erythrocyte glutathione (GSH) and plasma vitamin E, vitamin C, and uric acid concentrations was not affected by hydroxytyrosol supplementation. Moreover, the glutathione defence system in skeletal muscle was up-regulated by hydroxytyrosol, as shown by an elevation in GSH levels after exercise. Furthermore, the exercise-induced increase in glutathione reductase activity was even higher after hydroxytyrosol supplementation, compared to placebo. This indicates that hydroxytyrosol rather boosts than blocks the exercise induced adaptive response. Therefore, during exhaustive exercise, when the generation of ROS overwhelms the endogenous defensive mechanisms, hydroxytyrosol might be supplemented to strengthen the antioxidant capacity. Hydroxytyrosol did not attenuate the exercise-induced rise in urinary isoprostanes, indicating that hydroxytyrosol did not protect against oxidative stress. Hydroxytyrosol did, however, reduce the production of lactate during exercise. Irrespective of the mechanism involved, attenuation of lactate production during exercise by hydroxytyrosol is expected to enhance performance. 



\section{CHAPTER 8}

Summary and perspective

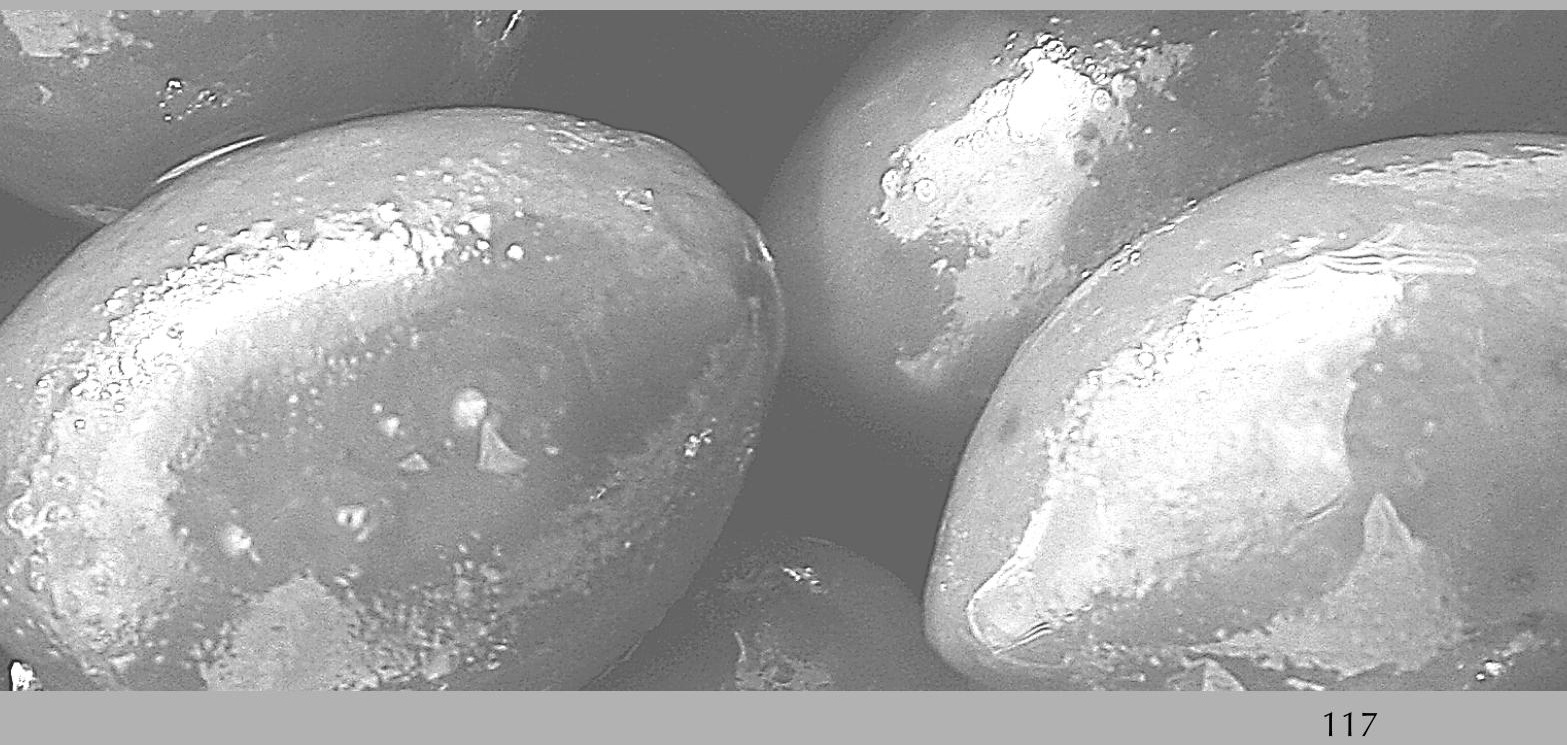




\section{SUMMARY}

Hydroxytyrosol is an excellent antioxidant. The fundament for the physiological impact of hydroxytyrosol is its high intrinsic antioxidant activity. Hydroxytyrosol is a potent scavenger of several reactive species, i.e. superoxide radical $\left(\mathrm{O}_{2}{ }^{--}\right)$, hydroxyl radical $\left(\mathrm{OH}^{\bullet}\right)$ and peroxynitrite $(\mathrm{ONOOH})$. The amphiphilic character of hydroxytyrosol facilitates its uptake in the intestines. Moreover, because of its amphiphilic nature, hydroxytyrosol is expected to provide protection in both lipid and aqueous cellular compartments.

Hydroxytyrosol has beneficial effects on cardiovascular health. It protects against the oxidative stress mediated impairment of blood vessel relaxation, thereby preserving one of the key mechanisms in regulating blood pressure. Moreover, the oxidation of low density lipoprotein (LDL) is efficiently prevented by hydroxytyrosol. The oxidation of LDL is regarded as one of the key steps of the initiation of atherosclerosis.

The applicability of hydroxytyrosol is not merely restricted to cardiovascular health. The present thesis shows that hydroxytyrosol efficiently protects against the oxidative stress mediated reduction in force production of isolated skeletal muscle. Furthermore, the supplementation of hydroxytyrosol in healthy men considerably boosts the antioxidant capacity of blood and muscle and attenuates the rise in plasma lactate during exercise. 


\section{PERSPECTIVE}

The application field of hydroxytyrosol is broad. Hydroxytyrosol is of interest in all kinds of situations involving oxidative stress. Based on the results described in this thesis, it would be interesting to further study the beneficial cardiovascular effects of hydroxytyrosol. Especially for people at risk (such as having a high blood pressure or high LDL to HDL ratio) hydroxytyrosol might be beneficial. With regard to the application of hydroxytyrosol, one should also be cautious by taking the law of Paracelsus into account. This law states that every compound, so also hydroxytyrosol, will be toxic provided that the dose is high enough. This applies especially to people not at risk taking supplements high in hydroxytyrosol over a long period of time. Therefore, it is important to establish not only the efficacy, but also the safety of long-term use of hydroxytyrosol. It should be noted that in the experiments described in this thesis, no signs of toxicity due to hydroxytyrosol administration were observed.

Another promising option is to further elaborate on the effect of hydroxytyrosol during exercise. The increase in TEAC of plasma lasted only approximately 90 minutes after intake of hydroxytyrosol. Supplementing hydroxytyrosol not only 30 minutes before, but also directly after exercise would have prolonged the increase in TEAC, which might have been more effective in protecting against oxidative damage. Here also specific target populations can be pointed out that might benefit most from hydroxytyrosol supplementation during exercise, for example people suffering from chronic obstructive pulmonary disease (COPD) or type 2 diabetes. Besides an impaired antioxidant defence, these patients have a relatively high production of radicals even under basal conditions. A negative health effect of a high radical flux induced by exercise is therefore expected to be more pronounced in these individuals. Interestingly, exercise is recommended in the treatment of these patients. Hydroxytyrosol supplementation during exercise might therefore especially be relevant for these patients, as it strengthens the antioxidant defence against ROS generated during exercise. 


\section{SAMENVATTING}

Hydroxytyrosol is een uitmuntende antioxidant. Het fundament voor de fysiologische impact van hydroxytyrosol wordt gevormd door de hoge intrinsieke antioxidant activiteit. Dit proefschrift laat zien dat hydroxytyrosol een effectieve scavenger is van verschillende reactieve deeltjes, zoals superoxide radicalen $\left(\mathrm{O}_{2}{ }^{*}\right)$, hydroxyl radicalen $\left(\mathrm{OH}^{*}\right)$ en peroxynitriet $(\mathrm{ONOOH})$. Het amphiphilisch karakter van hydroxytyrosol bevordert de opname van hydroxytyrosol in de darm. Bovendien wordt verwacht dat, vanwege het amphiphilisch karakter, hydroxytyrosol in staat is om als antioxidant bescherming te bieden in zowel hydrofobe als hydrofiele compartimenten van de cel.

Hydroxytyrosol heeft positieve cardiovasculaire gezondheidseffecten. Hydroxytyrosol is in staat om bescherming te bieden tegen de oxidatieve stress gemedieerde reductie in bloedvat relaxatie. Hierdoor blijft een essentieel mechanisme in de regulatie van de bloeddruk behouden. Daarnaast wordt de oxidatie van LDL effectief tegengegaan door hydroxytyrosol. De oxidatie van LDL wordt gezien als een van de belangrijkste stappen in de initiatie van atherosclerosis.

De toepasbaarheid van hydroxytyrosol is niet alleen beperkt tot cardiovasculaire gezondheid. Dit proefschrift toont aan dat hydroxytyrosol effectieve bescherming biedt tegen de oxidatieve stress gemedieerde reductie in krachtontwikkeling van geïsoleerd skeletspierweefsel. Daarnaast versterkt het supplementeren van hydroxytyrosol tijdens fysieke inspanning de antioxidant capaciteit van bloed en spieren van gezonde vrijwilligers. Bovendien verlaagt hydroxytyrosol de productie van melkzuur tijdens fysieke inspanning. 


\section{PERSPECTIEF}

Het toepassingsgebied van hydroxytyrosol is breed. Het supplementeren van hydroxytyrosol kan aantrekkelijk zijn in allerlei situaties waar oxidatieve stress een rol speelt. Het is interessant om de positieve cardiovasculaire gezondheidseffecten van hydroxytyrosol verder te onderzoeken. Met name voor risicogroepen (zoals mensen met een hoge bloeddruk of een hoge LDL/HDL ratio) zou hydroxytyrosol positieve gezondheidseffecten kunnen hebben. Men moet echter wel altijd de wet van Paracelsus in het achterhoofd houden. Deze wet zegt dat elke stof, dus ook hydroxytyrosol, toxisch is mits de dosis hoog genoeg is. Dit geldt in het bijzonder voor mensen zonder risicofactoren, die hydroxytyrosol supplementen in hoge dosering gebruiken gedurende lange tijd. Het is daarom van belang dat niet alleen de effectiviteit, maar ook de veiligheid van langdurig gebruik van hydroxytyrosol nader onderzocht wordt. In het onderzoek dat werd uitgevoerd in het kader van dit proefschrift werden geen tekenen van toxiciteit van hydroxytyrosol waargenomen.

Een andere veelbelovende mogelijkheid voor verder onderzoek is om het effect van hydroxytyrosol tijdens fysieke inspanning verder te ontrafelen. De toename in de totale antioxidant capaciteit van het bloed was waarneembaar tot ongeveer 90 minuten na inname van hydroxytyrosol. Deze relatief korte periode zou verlengd kunnen worden door meerdere malen hydroxytyrosol in te nemen. Hierdoor zou hydroxytyrosol mogelijk effectiever bescherming kunnen bieden tegen oxidatieve schade veroorzaakt door fysieke inspanning.

Er zijn situaties waarbij op voorhand verwacht kan worden dat hydroxytyrosol een positief gezondheidseffect heeft. Hierbij kan worden gedacht aan hydroxytyrosol supplementatie tijdens fysieke inspanning van patiënten met chronisch obstructief longlijden (COPD) of diabetes type 2 patiënten. Deze patiënten hebben, naast een verminderde antioxidant capaciteit, een hoge productie van radicalen onder normale omstandigheden. De door fysieke inspanning verhoogde productie van radicalen zou bij deze patiënten kunnen leiden tot meer geprononceerde negatieve gezondheidseffecten, in vergelijking met gezonde mensen. Het is opvallend dat fysieke inspanning juist aanbevolen wordt in de behandeling van deze patiënten. Hydroxytyrosol supplementatie tijdens fysieke inspanning zou daarom in het bijzonder relevant kunnen zijn voor deze patiënten, omdat dit de endogene antioxidant bescherming tegen de door fysieke inspanning gevormde radicalen kan versterken. 



\section{APPENDIX}

Dankwoord

About the author

\section{Publications}

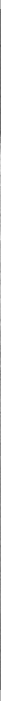




\section{DANKWOORD}

Promoveren doe je niet alleen. Graag wil ik iedereen bedanken die mij geholpen heeft om deze mijlpaal te bereiken.

Allereerst wil ik mijn promotor Aalt Bast en co-promotor Guido Haenen bedanken. Aalt, bedankt voor je grenzeloze interesse die je de afgelopen jaren getoond hebt, niet alleen in mijn onderzoek, maar ook in mij. Je enthousiaste houding ten opzichte van het verrichten van onderzoek werkte erg motiverend. De vele discussies die we samen met Guido gevoerd hebben, waren erg verhelderend en hielpen mij de rode draad van het onderzoek in zicht te houden. Ik heb me vaak verbaasd hoe gemakkelijk jij oplossingen kon bedenken voor de problemen waar ik tijdens het onderzoek tegenaan liep. Dat is iets waar ik je erg in bewonder. Bedankt voor de ruimte die je me gaf om me verder te kunnen ontwikkelen!

Guido, bedankt voor je hulp bij alle facetten van het onderzoek. Je leerde mij mijn sterke punten te benadrukken en mijn zwakke punten te accepteren, hiervoor ben ik je erg dankbaar. Ik denk nog vaak terug aan de vele structuurschetsen die we maakten voor de verschillende artikelen en hoe we uiteindelijk, na veel puzzelen, tot een juiste opzet kwamen. Jouw deur stond voor mij altijd open, en dat heb ik altijd erg gewaardeerd. Ook bedankt voor de hulp bij de laatste fase, het afronden van het proefschrift. De laatste loodjes hebben hierdoor zeker minder zwaar gewogen!

Verder wil ik alle collega's van de afdeling Farmacologie en Toxicologie bedanken voor de gezellige werksfeer. In het bijzonder wil ik graag Marc en Marie-José bedanken voor de hulp bij de proefpersonenstudie en bij de analyses op het lab. Bedankt voor jullie flexibiliteit en inzet! Natuurlijk wil ik ook de proefpersonen bedanken, die grote fysieke inspanningen hebben geleverd tijdens het "sportieve" gedeelte van mijn studie. Ook bedank ik Domenique Nijsten voor haar inzet als stagiaire op mijn project. De collega's van Bewegingswetenschappen en Humane Biologie, Milou Beelen, René Koopman, Ralph Manders en Luc van Loon, wil ik graag bedanken voor hun hulp bij de proefpersonenstudie. Ik vond het erg prettig en leerzaam om met jullie samen te werken, en heb via deze weg een kijkje kunnen nemen in een heel ander vakgebied. Ook wil ik Bart Pennings en Nico Crombach bedanken voor hun praktische hulp bij de proefpersonenstudie en natuurlijk voor hun bijdrage als professionele acteurs tijdens de filmopnames! Jan de Vente wil ik graag bedanken voor zijn hulp bij de kleuringen van de aorta weefsels. Ook dank aan Ronald Deumens voor de weefsels die ik kon gebruiken voor mijn ex vivo studies. Op deze manier zijn er heel wat minder proefdieren voor mijn onderzoek nodig geweest, en dat is iets wat ik erg waardevol acht. 
Mijn nieuwe collega's in Utrecht bedank ik voor de interesse die zij getoond hebben tijdens de afrondingsfase van mijn proefschrift en voor de prettige werkomgeving die zij mij bieden. Ik voel me echt thuis bij jullie op mijn nieuwe werkplek!

Uiteraard wil ik ook graag mijn vrienden bedanken. Wonend in verschillende steden heb ik gemerkt dat "uit het oog" niet per definitie "uit het hart" betekent. ledereen bedankt voor jullie gezelligheid en steun de afgelopen jaren. Ook dank aan mijn vrienden in Utrecht; zonder jullie zou ik me niet zo snel thuis gevoeld hebben in deze nieuwe stad!

In het bijzonder wil ik een speciaal woord van dank uitspreken aan een aantal vrienden. Liesbeth, heel erg bedankt voor je hulp op alle fronten, zowel op het werk als privé. Ik vind het heel bijzonder dat we in Utrecht opnieuw collega's geworden zijn. Ik zie er naar uit om de komende tijd Utrecht verder te ontdekken. Binnenkort weer een fietstochtje maken? Monica, ook al heeft jouw leven ineens een andere wending genomen en zien we elkaar niet meer zo vaak als vroeger, ik weet dat ik bij jou altijd terecht kan. Ik ben trots op jou en op de manier waarop jij in het leven staat. Jantine, bedankt voor je hulp met het ontwerpen van de kaft. Ik ben erg blij met het uiteindelijke resultaat!

Natuurlijk mogen mijn paranimfen in dit lijstje niet ontbreken. Ik vind het heel fijn dat jullie tijdens mijn verdediging, letterlijk en figuurlijk, achter me staan. Hanneke, wij kennen elkaar al nu al zo lang. Onze levens hebben altijd parallel gelopen, nu wonen we in verschillende steden, maar ik weet zeker dat we elkaar nooit uit het oog zullen verliezen. Bedankt voor je altijd nuchtere blik op de wereld!

Judith, als "ervaringsdeskundige" weet jij natuurlijk precies wat het inhoudt om te promoveren en welke moeilijkheden dat met zich mee kan brengen. Het is fijn om te weten dat ik bij jou altijd kan aankloppen voor goed advies, bedankt daarvoor!

Mijn grootste dank gaat natuurlijk uit naar mijn ouders. Pap, mam, bedankt voor jullie altijd aanwezige belangstelling en onvoorwaardelijke steun bij alles wat er op mijn pad komt. 


\section{ABOUT THE AUTHOR}

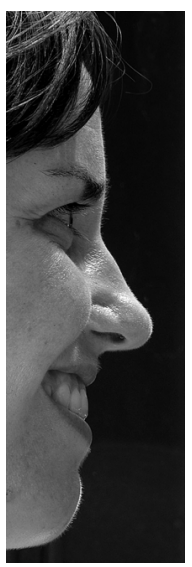

Saskia J. Rietjens was born on July 12th 1980 in Roermond. Her secondary school period took place at 'Bisschoppelijk College Broekhin' in Roermond, where she received her gymnasium certificate in 1998.

From 1998-2003 she studied Biomedical Health Sciences at Radboud University Nijmegen with a specialization in Toxicology. Experience in clinical research was obtained during an internship at the department of Internal Medicine of University Medical Centre St Radboud. Additional research experience was obtained during an internship at the Nephrology Research Laboratory, Nijmegen Centre for Molecular Life Sciences, University Medical Centre St Radboud.

In September 2002, she moved to Maastricht to specialize in Nutritional Toxicology. Her MSc thesis entitled 'Oxidative stress: damage and protection' was based on research conducted at the department of Pharmacology and Toxicology of Maastricht University. After obtaining her MSc in 2003, she continued her work as a PhD-student at the same department, under the supervision of Dr. Guido Haenen and Prof. dr. Aalt Bast, resulting in the present thesis. During this period, she also completed the Postgraduate Education in Toxicology, which will result in the registration as Toxicologist.

From September 2007 onward, she works as researcher and consultant in Clinical Toxicology at the National Poisons Information Centre of the National Institute for Public Health and the Environment (RIVM), Bilthoven. 


\section{PUBLICATIONS}

de Galan BE, Rietjens SI, Tack CJ, van der Werf SP, Sweep CG, Lenders JW, Smits $\mathrm{P}$. Antecedent adrenaline attenuates the responsiveness to but not the release of counterregulatory hormones during subsequent hypoglycemia. / Clin Endocrinol Metab 2003;88(11):5462-7.

Vogtländer NP, Rietjens SI, van der Vlag J, Berden JH. Podocyte dysfunction and proteinuria. Ned Tijdschr Geneeskd 2004;148(15):712-8.

Michels G, Haenen GR, Watjen W, Rietjens SI, Bast A. The thiol reactivity of the oxidation product of 3,5,7-trihydroxy-4H-chromen-4-one containing flavonoids. Toxicol Lett 2004;151(1):105-11.

Schouwenberg BJ, Rietjens SI, Smits P, de Galan BE. Effect of sex on the cardiovascular response to adrenaline in humans. J Cardiovasc Pharmacol 2006;47(1):155-7.

Rietjens SI, Bast A, de Vente J, Haenen GR. The olive oil antioxidant hydroxytyrosol efficiently protects against the oxidative stress induced impairment of the NO ${ }^{*}$ response of isolated rat aorta. Am / Physiol Heart Circ Physiol 2007;292(4):H1931-6.

Rietjens SI, Bast A, Haenen GR. New insights into controversies on the antioxidant potential of the olive oil antioxidant hydroxytyrosol. I Agric Food Chem 2007;55(18):7609-14.

Rietjens SI, Beelen M, Koopman R, van Loon LJ, Bast A, Haenen GR. A single session of exercise induces oxidative damage in untrained men. Med Sci Sports Exerc 2007;39(12):2145-51.

Rietjens SI, Bast A, Haenen GR. The olive oil antioxidant hydroxytyrosol protects against oxidative stress-induced reduction in skeletal muscle force production. Submitted, 2008.

Rietjens SI, Beelen M, Koopman R, van Loon LJ, Bast A, Haenen GR. The olive antioxidant hydroxytyrosol boosts the endogenous antioxidant defence and attenuates the rise in plasma lactate during resistance type exercise. Submitted, 2008. 
